\title{
Neuroligins Determine Synapse Maturation and Function
}

\author{
Dissertation \\ zur Erlangung des Doktorgrades \\ der Mathematisch-Naturwissenschaftlichen Fakultäten \\ der Georg-August-Universität Göttingen
}

\author{
vorgelegt von \\ Gayane Aramuni \\ aus Echmiadzin, Armenien
}

Göttingen 2007 
D7

Referent: Prof. Dr. Ralf Heinrich

Koreferent: Prof. Dr. Michael Hörner

Tag der mündlichen Prüfung: 
Dedicated to my Parents 


\section{Contents}

\section{Abbreviations}

1. Introduction 1

1.1. Neurotransmission 1

$1.2 \quad$ Localization and function of preBötzinger complex

1.3. Neuroligins as cell-adhesion molecules 3

1.3.1 Structural features of neuroligins 3

1.3.2 Expression of neurologins 4

1.3.3 Binding partners of neuroligins 4

$1.4 \quad$ NLs and functional deficiencies in human brain 6

$1.5 \quad$ The role of NLs in synaptogenesis and synaptic function 6

1.6 Aims of the present study $\quad 8$

2. Materials and methods 9

2.1 Electrophysiology 9

2.1.1 Animals 9

2.1.2 Brain slice preparation 9

2.1.3 Solutions and drugs used for electrophysiology 10

2.1.4 Electrophysiological recordings 12

2.1.5 Capacitance and series resistance calculation 13

2.1.6 Recordings of spontaneous and evoked postsynaptic currents 14

2.1.7 Ventilation recordings 15

2.1.8 Data analysis 15

$2.2 \quad$ Fluorescence immunohistochemistry 15

2.2.1 Brain tissue preparation for immunohistochemsitry 15

2.2.2 Solutions and chemicals 16

2.2.3 Antibodies used for immunofluorescence labeling 17

2.2.4 Immunofluorescence staining 17

$\begin{array}{lll}2.2 .5 & \text { Data analyses } & 18\end{array}$ 
$\begin{array}{lll}2.3 & \text { Biochemical methods } & 18\end{array}$

2.3.1 Solutions, antibodies used for biochemical procedures 18

2.3.2 Primary and secondary antibodies 20

2.3.3 Protein extracts preparation 20

2.3.4 Protein concentration estimation 21

2.3.5 SDS-polyacrilamide gel electrophoresis (SDS-PAGE) 21

2.3.6 Western blotting 23

3. Results 24

Article: Neuron 51, 741-754 (2006)

"Neuroligins Determine Synapse Maturation and Function"

3.1 Ventilation patterns measured by whole-body plethysmography 42

3.2 Synaptic transmission in brainstem preBötzinger complex in $\quad 42$ neuroligin 2 mutant mice

3.2.1 Decreased spontaneous postsynaptic currents in brainstem PBC 44 neurons of NL 2 mutant mice

3.2.2 Impaired inhibitory synaptic transmission in NL 2 KO mice 44

3.2.3 Deletion of NL 2 causes severe reduction of GABAergic synaptic 49 transmission

3.2.4 Glycinergic synaptic transmission is affected in NL 2 KO mice 52

3.2.5 Unaltered excitatory synaptic transmission in PBC of mice lacking 56 NL 2

3.3 Evoked neurotransmitter release in NH in NL 2 deficient mice 59

3.3.1 Lack of neuroligin 2 slightly decreases electrically evoked 60 synaptic transmission in hypoglossal neurons

3.3.2 Electrically evoked inhibitory neurotransmission is affected in 61 neuroligin 2 mutant mice

3.3.3 Evoked EPSCs is not different between control and NL 2 KO 63 mice

3.4 Synaptic protein expression and synaptogenesis in brainstem of $\quad 65$ NL 2 KO mice 
3.4.1 The expression of synaptic proteins in brainstem of NL 2 KO 65 mice

3.4.2 Synaptogenesis in respiratory brainstem of NL $2 \mathrm{KO}$ mice

4. Discussion $\quad 71$

4.1 Neuroligin triple knockout mice $\quad 71$

4.1.1 Synaptogenesis in mice lacking NLs 1-3 71

4.1.2 The role of NLs 1-3 in synaptic function 73

4.2 The effects of NL 2 deletion in vivo 76

4.2.1 Loss of NL 2 does not alter synaptogenesis in PBC 76

4.2.2 Excitatory synaptic activity remains unaltered in NL 2 KO mice $\quad 77$

4.2.3 Deletion of NL 2 strongly impairs GABAergic/glycinergic 78 transmission

$\begin{array}{ll}\text { 5. Summary } & 80\end{array}$

$\begin{array}{lll}\text { 6. } & \text { References } & 82\end{array}$

$\begin{array}{lll}\text { 7. Acknowledgments } & 89\end{array}$

$\begin{array}{lll}\text { 8. Curriculum Vitae } & 91\end{array}$

$\begin{array}{ll}\text { 9. } & 92\end{array}$ 


\section{Abbreviations}

ACSF

AMPA

APS

ATP

$\mathrm{Bp}$

CNS

CNQX

DKO

DL-AP5

EDTA

eEPSCs

elPSCs

et al.

g

GABA

$\mathrm{G} \Omega$

HEPES

$\mathrm{kDa}$

$\mathrm{kHz}$

$\mathrm{KO}$

$\mu-$

mEPSCs

mIPSCs

$\mathrm{mV}$

NA

NGS

$\mathrm{NH}$
Artificial cerebrospinal fluid

a-Amino-3- hydroxyl-5-methyl-4-isoxazole propionic acid

Ammonium persulfate

Adenosine tryphosphate

base pair

Central nervous system

6-cyano-7 nitroquinoxaline-2, 3-dione disodium salt

Double knockout

DL-2-Amino-5-phosphonovaleric acid

Ethylenedinitrilo-tetraacetic acid

evoked excitatory postsynaptic currents

evoked inhibitory postsynaptic currents

et alters (and others)

Gravitational acceleration, or gram

Y-aminobutyric acid

\section{Giga Ohm}

4-(2-Hydroxyethyl) piperazine-1-ethanesulfonic acid Kilodalton

kilo Hertz

Knockout

micro, $-\left(x^{10} 0^{-6}\right)$

miniature excitatory postsynaptic currents

miniature inhibitory postsynaptic currents

millivolts

Nucleus ambiguus

Normal goat serum

Nucleus hypoglossus 


\begin{tabular}{|c|c|}
\hline $\mathrm{NL}$ & Neuroligin \\
\hline NMDA & N-methyl-D-Aspartate \\
\hline N-terminal & at the $\mathrm{NH}$-terminus of a protein \\
\hline $\mathrm{pA}$ & pico Ampere \\
\hline PAGE & Polyacrylamide gel electrophoresis \\
\hline PBC & PreBötzinger complex \\
\hline PBS & Phosphate buffer saline \\
\hline PFA & paraformaldehyde \\
\hline $\mathrm{pH}$ & Negative logarithm of $\mathrm{H}+$ concentration \\
\hline PDZ & $\begin{array}{l}\text { Protein interaction domain, acronym for PSD-95, Dlg, } \\
\text { ZO-1 }\end{array}$ \\
\hline ProsAP & praline-rich synapse-associated protein \\
\hline PSD & postsynaptic density \\
\hline RT & Room temperature \\
\hline s & second \\
\hline SDS & Sodium dodecyl sulfate \\
\hline sEPSCs & spontaneous excitatory postsynaptic currents \\
\hline sIPSCs & spontaneous postsynaptic currents \\
\hline SKO & Single knockout \\
\hline sPSCs & spontaneous postsynaptic currents \\
\hline TKO & Triple knockout \\
\hline Tris & Tris-hydroxymethyl-aminomethane \\
\hline TTX & Tetrodoxin \\
\hline VIAAT & vesicular inhibitory amino acid transporter \\
\hline vGlut & vesicular glutamate transporter \\
\hline WT & Wild type \\
\hline $5^{\prime}$ & 5 prime \\
\hline
\end{tabular}




\section{Introduction}

\subsection{Neurotransmission}

The mammalian brain is a highly specific neuronal network, where billion of neurons are connected through synapses, which are specialized intrinsic asymmetric contacts, mediating precise, rapid and efficient communication between neurons. Synapses are classified into two types: chemical and electrical. Most synapses in the nervous system are chemical. Typically they are consisted of presynaptic and postsynaptic compartments, separated by synaptic cleft. Presynaptic compartment usually is localized on axons, while postsynaptic specialization can be localized on dendrites, soma and in some cases on axon. Presynaptic specialization of the synapse is characterized by neurotransmitterloaded synaptic vesicles at active zone and required for neurotransmitter release into synaptic cleft, whereas the postsynapse, which contains receptors, ion channels and associated proteins (post-synaptic density), is specialized for signal transduction. Cell adhesion molecules (CAMs) (Serafini, 1999; Yamagata et al., 2003) bridge the pre- and postsynaptic compartments of synapses in the central nervous system. They can promote differentiation of pre- and postsynaptic specializations and take a part in regulation of synaptic structure and function (Yamagata et al., 2003). They are several families of cell adhesion molecules that have been discovered (Brose, 1999; Missler et al., 2003; Scheiffele, 2003; Yamagata et al., 2003).These membranous proteins, present on pre- and postsynaptic membranes, are in a heterophilic or homophilic interaction with their partners on the opposite side of synaptic cleft.

Neurotransmitters are released in a process termed exocytosis. This process is initiated upon an arrival of an action potential at the presynaptic terminal, which generates depolarization of presynpase, leads to the opening of voltage gated $\mathrm{Ca}^{2+}$ channels at the active zones and $\mathrm{Ca}^{2+}$ ions entering into the presynaptic terminal (Zucker et al, 1993). The rise in $\mathrm{Ca}^{2+}$ concentration brings the readily releasable vesicles to fuse with the plasma membrane and release their 
neurotransmitter into the synaptic cleft (Landis et al., 1988; Dresbach et al., 2001; Harlow et al., 2001). The transmitter molecules then diffuse across the synaptic cleft, bind to their specific receptors on the postsynaptic compartment of synapse and activate them, leading to the opening and or closing of ion channels.

\subsection{Localization and function of the preBötzinger complex}

The respiratory rhythm originates from special neuronal regions located in restricted areas in the reticular formation of the ventrolateral medulla. One of these regions is bilaterally localized preBötzinger complex (PBC), which contains all classes of respiratory neurons essential for respiratory rhythm generation (Smith et al., 1991; Connely et al., 1992; Schwarzacher et al., 1995; Feldman et al., 2003). PreBötzinger complex is located ventro-lateral to the nucleus ambiguus (NA), caudal to the retrofacial nucleus, and rostral to the anterior tip of the lateral reticular nucleus (Bregma-level $-12.0 \mathrm{~mm}$ to $-12.5 \mathrm{~mm}$ ) (Gray et al., 1999; Guyenet and Wang, 2001; Wang et al., 2001) (Fig.1.1).
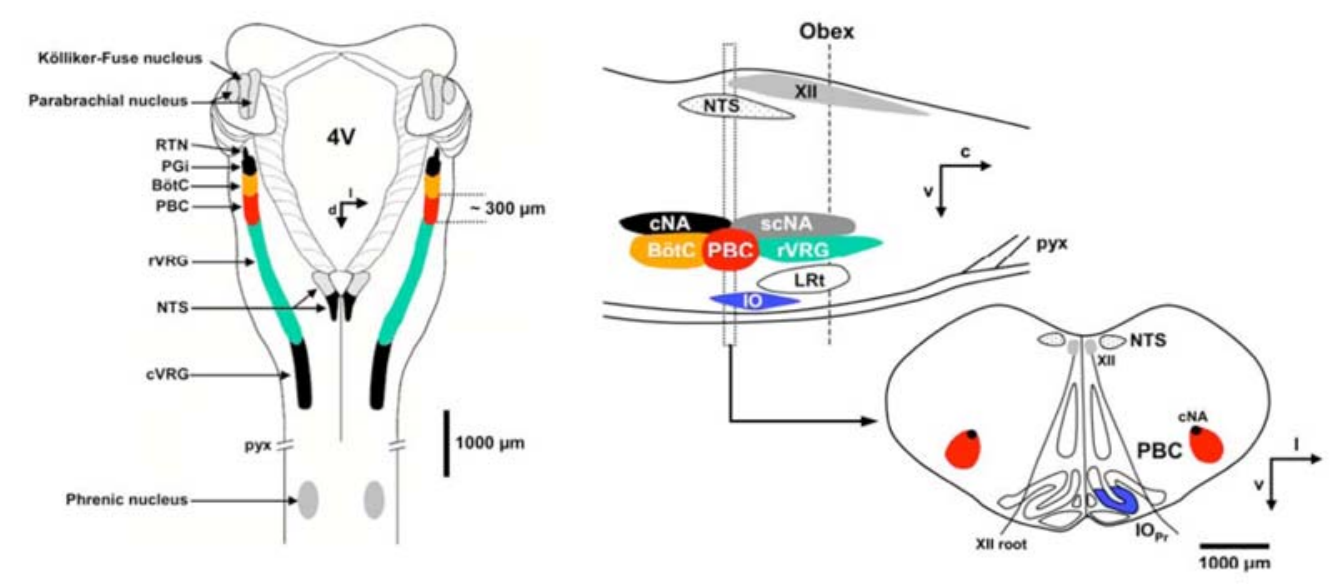

Figure 1.1 Schematic representation of the localization of preBötzinger complex in rat brainstem: On the left side a dorsal topview of the rat brainstem after removal of the cerebellum is shown. On the right side a sagittal section (top) and a transversal slice (bottom), which contains the PBC, are shown. Abbreviations: nucleus solitary tract (NTS), hypoglossal nucleus (XII), nucleus ambiguus, compact part (cNA), preBötzinger complex (PBC), principal nucleus of the inferior olive $\left(\mathrm{IO}_{\mathrm{Pr}}\right)$. 
The nucleus ambiguus and principal nucleus of the inferior olive $\left(\mathrm{IO}_{\mathrm{Pr}}\right)$ help for anatomical identification of PBC. The neurons of PBC are immunoreactive for the neurokinin receptor neurokinin 1 (NK1) (Gray et al., 1999; Wang et al., 2001) and the destruction of neurons expressing NK1 leads to an ataxic respiration (Gray et al., 2001), which together with other experiments (Pierrefiche et al., 1998; Smith et al., 1991; Solomon et al., 1999) confirms the view that the preBötzinger complex plays a primary role in the respiratory rythmogenesis.

\subsection{Neuroligins as cell-adhesion molecules}

Neuroligins constitute a family of cell adhesion proteins that interact with presynaptic $\alpha$ - and $\beta$-neurexins and form heterotypic intercellular junctions. Neuroligin proteins have been identified in humans, rodents, chicken, drosophila melanogaster and Caenorhabditis elegans (Ichtchenko et al., 1995, 1996; Kwon et al., 2004; Gilbert et al., 2001; Bolliger et al., 2001; Paraoanu at el., 2005). Four members of neuroligin family are characterized in rodents and five genes coding for neuroligins have been identified in the human genome (Boucard et al., 2005, Ichtchenko et al., 1995, Song et al., 1999, Varoqueaux et al., 2004).

\subsubsection{Structural features of neuroligins}

All neuroligin isoforms have large extracellular acetilycholinesterase-like domain, which lacks cataitic activity and binds to neurexins. It is followed by transmembrane segment and shorter intracellular terminal PDZ binding motif. All neuroligins are subject to alternative splicing at two conserved splice sites, abbreviated to as A and B (Fig.1.2) that are distinct for neuroligin 1 and 2, while neuroligin 3 contains two types of alternatively spliced variants, one is similar to that present in neuroligin 1, and the second is a hybrid of sequences present in neuroligin 1 and neuroligin 2 (Ichtchenko et al., 1995, 1996). The existence of two alternatively spliced regions allows the generation of up to four different isoforms for each neuroligin gene (Ichtchenko et al., 1996; Boucard et al., 2005). 


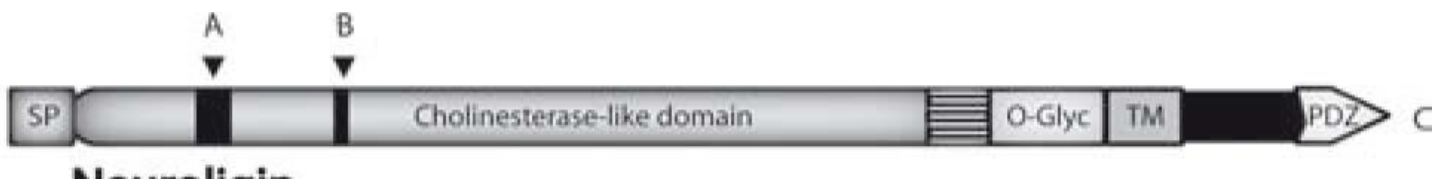

\section{Neuroligin}

Figure 1.2 The domain structure of neuroligins. The N-terminal extracellular region of neuroligins is composed of a signal peptide (SP), followed by cholinesterase-like domain, which mediates binding to neurexin LNS domain. It contains two alternatively spliced sites (A and B), an oligomerization domain (hatched box) and a carbohydrate attachment region for O-linked glycosylation (O-glyc.). Five N-glycosylation sites and two EF-hand motifs involved in $\mathrm{Ca}^{2+}$ binding are also present in the extracellular domain of neuroligins (not shown).

\subsubsection{Expression of neurologins}

Neuroligins are expressed throughout brain with differential and overlapping distribution of different isoforms. As described in different studies, mRNAs of neuroligins 1-3 are expressed at low levels in newborn rat and mouse brain and their expression increases (2- to 3-fold) during postnatal development (Song et al., 1999; Varoqueaux et al., 2004). NL 1 is expressed in central nervous system and localized specifically at the excitatory synapses (Song et al., 1999; Prange et al., 2004), whereas NL 2 is concentrated at the postsynaptic membranes of inhibitory synapses (Varoqueaux et al., 2004, Graf et al., 2004) and presents also in other tissues such as pancreas, lung, endothelia, uterus and colon. NL 3 expression except neurons has also been found in different glial cells (Philibert et al., 2000). In newborn mice NL 4 is not detectable, and in adult mice, only $3 \%$ of the total NL proteins in brain are contributed by NL 4 . In humans NL 4 is detected in heart, liver, skeletal muscle, pancreas, and at low levels in the brain. The existence of fifth neuroligin gene has also been reported (Bolliger et al., 2001; Jamain et al., 2003). NL 5 also referred as neuroligin $4 \mathrm{Y}$ because of its localization on the $\mathrm{Y}$ chromosome, differs from $\mathrm{X}$ linked NL 4 by only 19 amino acids.

\subsubsection{Binding partners of neuroligins}

There are some interaction partners for neuroligins that have been discovered. Extracellularly neuroligins bind to $\alpha$ - (Ichtchenko et., al 1995, 1996) and $\beta$ - 
neurexins (Boucard et al., 2005). The interaction between neuroligins and neurexins mediates trans-synaptic adhesion (Nguyen and Südhof, 1997). Postsynaptically, the intracellular tail of neuroligin isoforms binds to several PDZ domain-containing PSD-95, SAP-102, PSD-93 (Irie et al., 1997) and S-SCAM (Hirao et al., 1998) scaffolding proteins of glutamatergic postsynapses, which in turn interact with postsynaptic transmitter receptors, ion channels and signaling proteins (Hirao et al., 1998, Irie et al., 1997, Meyer et al., 2004). In contrast to glutamatergic synapses so far there are not direct experimental evidences for molecular composition of the postsynaptic specialization in GABAergic synapses and therefore the binding partners that are involved for targeting neuroligin 2 to inhibitory synapses are not clearly known (Varoqueaux et al., 2004). In Figure 1.3 are illustrated well known and proposed postsynaptic binding partners of neuroligins at glutamatergic and GABAergic postsynapses.

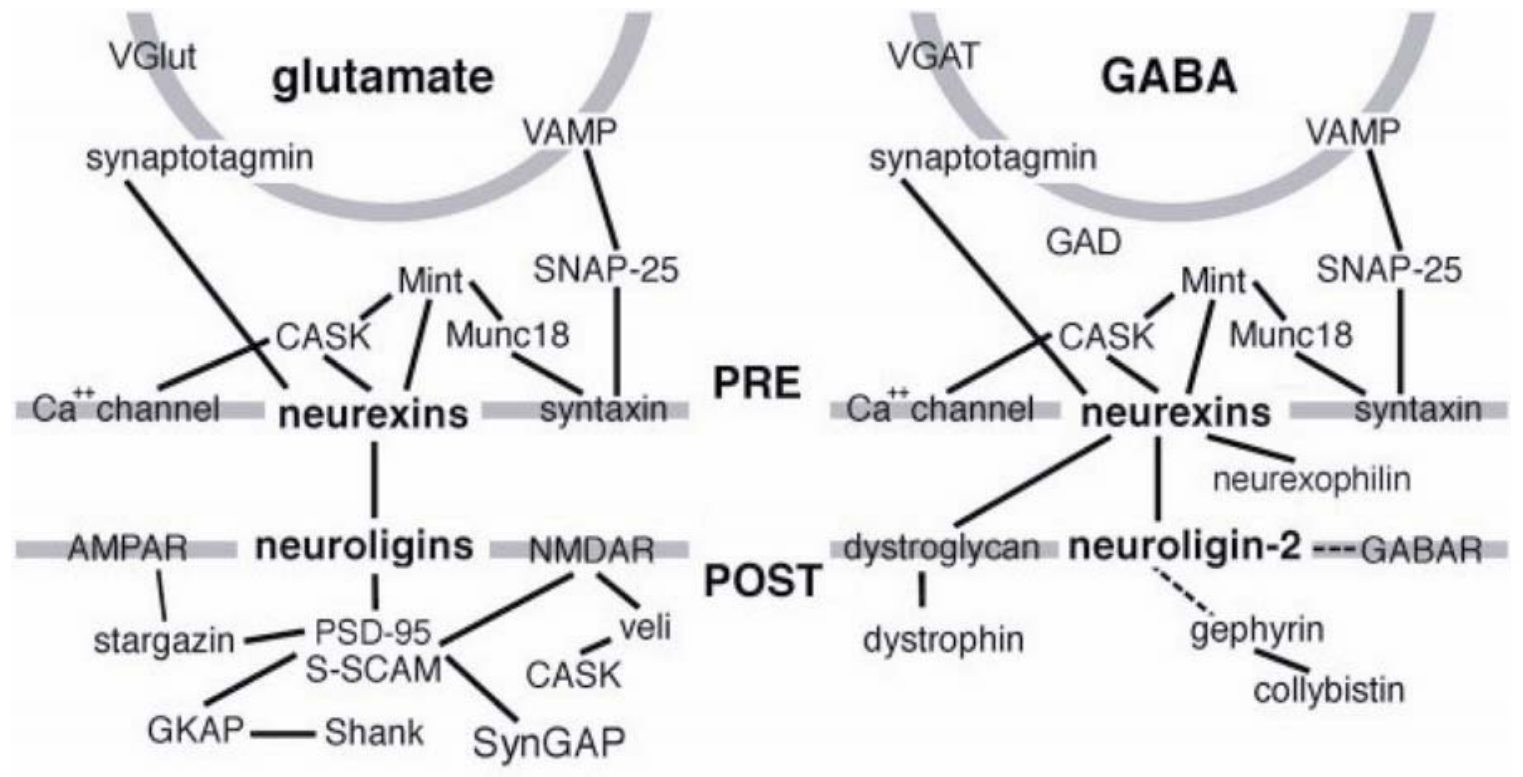

Figure 1.3 Binding partners of neuroligins at glutamatergic and GABAergic synapses. This model shows many interactions of neuroligins with different proteins at glutamatergic postsynapse. In contrast, binding partners of neuroligin 2 at GABAergic postsynapses remain unknown. Lines indicate reported protein-protein interactions and dashed lines show proposed, most likely indirect, interactions. 


\subsection{Neuronal disorders associated with dysfunction of neuroligins}

Autism is a complex neuro-developmental disorder characterized by severe reciprocal social interaction and communication impairment, which is often associated with mental retardation and epilepsy. Autistic individuals have larger brains, although the cerebral cortex, hippocampus and amygdale are smaller (Herbert et al, 2003; Aylward et al., 1999). The male-to-female ratio is $4: 1$ in autism. Twin studies and high number of male autistic patients provide the evidence for a genetic origin of autism (Beaudet et al., 2002). Many studies support the hypothesis that mutations in human neuroligin genes NL 3 and NL 4 cause rare monogenic heritable forms of autism and mental retardation (Chih et al., 2004; Comolleti et al., 2004; Jamain et al., 2003; Laumonnier et al., 2004; Yan et al., 2005; Talebizadeh et al., 2006), as are perturbances in the excitatory to inhibitory ratio and morphological aberrations in dendritic spines (Kaufmann et al., 2000; Hussman et al., 2001; Zoghbi., 2003). Therefore mice lacking neuroligin genes could be useful animal model for studying pathophysiologic mechanisms in autism.

\subsection{The role of NLs in synaptogenesis and synaptic function in vitro}

During development, synapse assembly is crucial highly controlled process in central nervous system that is initiated by contact formation between axon growth cone and target cell. Initial contact is followed by coordinated differentiation and maturation of the pre- and postsynaptic sites. Both processes seem to be driven by several classes of cell adhesion molecules (CAMs). Several recent studies performed in neuronal cultures have shown the potential role of neuroligins as CAMs and their binding partners in the formation and maturation of excitatory and inhibitory neuronal synaptic contacts (Chih et al., 2005; Dean et al., 2003; Graf et al., 2004; Levinson et al., 2005; Prange et al., 2004; Scheiffele et al., 
2000; Garner et al., 2002; Waites et al., 2005; Craig et al., 2006; Dean et al., 2006). Overexpression of NLs in cultured neurons increases the number of synapses (Chih et al., 2005; Dean et al., 2003; Graf et al., 2004; Levinson et al., 2005; Nam and Chen, 2005; Prange et al., 2004) and knock-down of neuroligin 1, 2 and 3 expression either individually or collectively by RNAi causes reduction in both excitatory and inhibitory presyanptic terminals (Chih et al., 2005). For example, the data obtained from mouse hippocampal neurons (Levinson et al., 2005) demonstrate that neuroligins drive excitatory and inhibitory presynaptic contact formation and various members of neuroligin family are able to exert similar effects on formation of new synaptic contacts (Fig.1.4). These studies demonstrate the possible involvement of these cell adhesion molecules, in particular NL 2, in controlling the balance between excitatory and inhibitory synapses. Simultaneous or individual overexpression and knock-down of neuroligins in cultured neurons leads to apparent changes in synaptic activity, which were explained by the dramatic effects of these manipulations on synapse numbers (Graf et al., 2004, Chih et al., 2005).
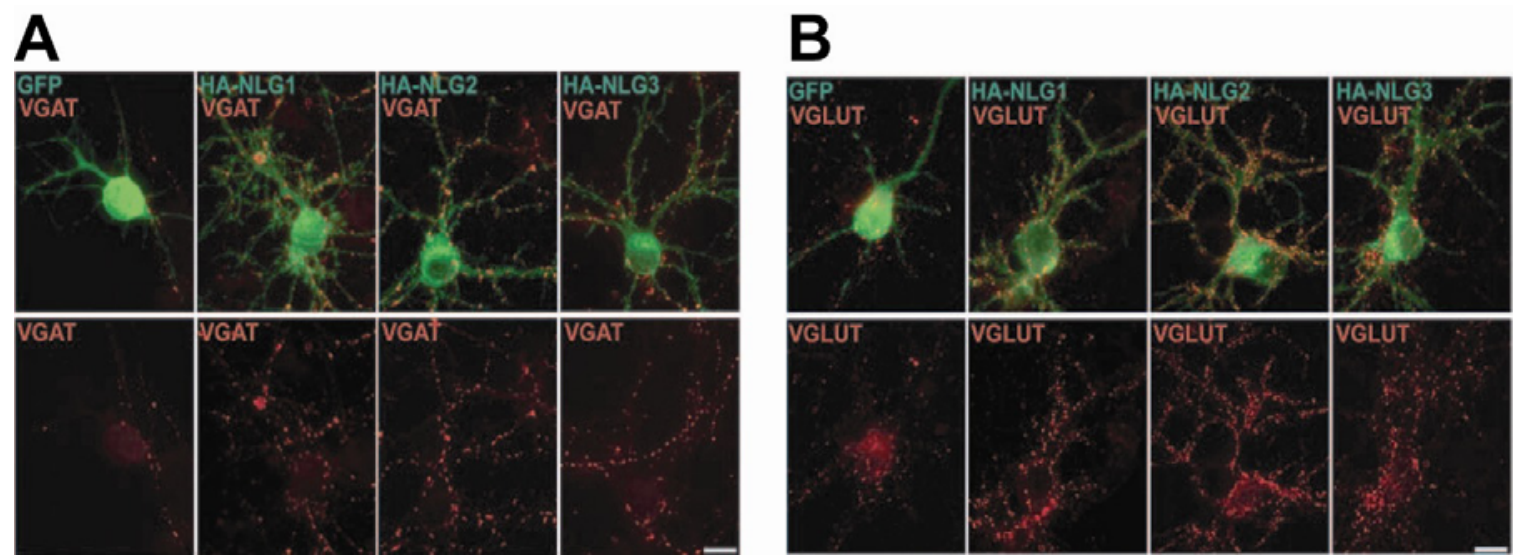

Figure 1.4 DIV 5 hippocampal neurons transfected with HA-NL1, HA-NL2 or HA-NL3 and then fixed at DIV 8 have increased VGAT (A) and VGLUT(B) positive puncta as compared to GFPtransfected cells. 
In vivo experiments on animals mutant for all known neuroligins may help to clarify the role of these proteins in synaptic function and different aspects of synapse development, including contact initiation, target recognition, synapse stabilization/maturation (Lise and El-Husseini, 2006).

\subsection{Aims of the present study}

The present work aims at investigating the role of neuroligins in synaptogenesis, and synapse function in the respiratory brainstem of mouse. For this purpose knockout mice were generated that had deletion of one, two or three neuroligins. The neuronal network of mouse respiratory brainstem, which is almost mature at birth time, is an appropriate system to investigate the role of neuroligins in vivo. The project is subdivided into two main steps. First step is the investigation of synaptogenesis and synaptic activity in NL 1-3 triple knockout mice, using molecular biological and electrophysiological approach. Because of early postnatal death of NL 1-3 triple KOs, experiments were performed on newborn mice. Second step contains detailed study of consequences of NL 2 deletion in synaptogenesis and synaptic transmission in brainstem respiratory network. For this, experiments were done in acute brainstem slices derived from neuroligin 2 knockout mice, using combined electrophysiological, immunohistochemical and biochemical methods. 


\section{Materials and methods}

\subsection{Electrophysiology}

\subsubsection{Animals}

Experiments were performed on NL 1-3 and NL 2 knockout mice. To generate mice that are lacking NLs 1-3, exon sequences covering the translational start site and at least $380 \mathrm{bp}$ of $5^{\prime}$ coding sequence of the respective genes were deleted by homologous recombination in embryonic stem cells. KOs lacking individual NLs and all combinations of double KOs (DKO 1/2, DKO 1/3 and DKO 2/3) were generated by interbreeding and were obtained at the predicted Mendelian frequencies. NL 1-3 and NL 2 KO mice were generated in and generously supplied by the lab of Prof. N. Brose. Animal experiments were carried out in accordance with the guidelines of the Ethics committee of the University of Göttingen.

\subsubsection{Brain slice preparation}

The preparation of transverse brainstem slices containing pre-Bötzinger complex (PBC) and hypoglossal nucleus (NH) followed the general procedure of making thin slices from the tissue of mouse central nervous system described in (Zhang et al., 1998). Postnatal day 0 (P0) to P5 male or female littermate mice were decapitated at C3-C4 spinal level. The whole brain was carefully removed from the cut-open skull, immediately transferred into ice-cold artificial cerebrospinal fluid (ACSF), which was already bubbled with carbogen $\left(95 \% \mathrm{O}_{2}\right.$ and $\left.5 \% \mathrm{CO}_{2}\right)$. The brainstem was separated from the cerebellum and forebrain. Transverse 200 $\mu \mathrm{m}$-thick slices were cut using a vibratome slicer (752M Vibroslice, Campden IInstruments, UK). Sectioning of the brainstem was done from the rostral to caudal part and fourth ventricle was used as a marker for the start of the region of interest. After sectioning, each slice was quickly placed into an incubation chamber containing aerated ACSF. Slices were kept at $28-30^{\circ} \mathrm{C}$. 


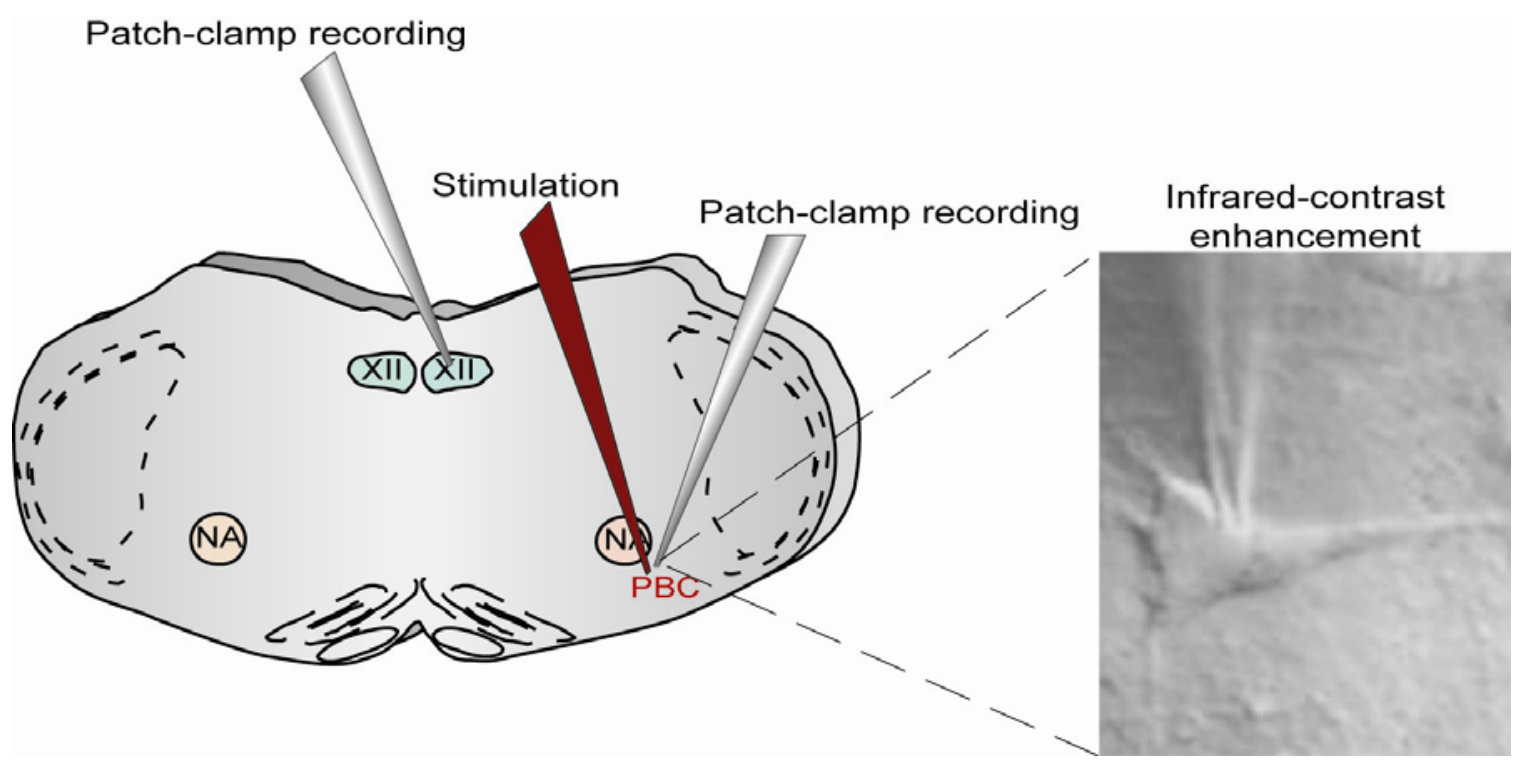

Figure 2.1 Schematic representation of respiratory brainstem slice (200 $\mu \mathrm{m}$ thickness) used for electrophysiological recordings. Abbreviations: preBötzinger complex (PBC), nucleus ambiguus (NA), hypoglossal nucleus (NH).

\subsubsection{Solutions and drugs used for electrophysiology}

The standard (Ringer) extracellular solution (Table 2-1) was used for brain slice preparations and electrophysiological recordings. It had a $\mathrm{pH}$ of 7.4 and an osmolarity of about 310 mOsm. The following tables (Table 2-2; 2-3) describe the compositions of different intracellular (patch-pipette) solutions. For recordings of spontaneous postsynaptic currents as an intracellular solution INK was used (Table 2-2) and to record evoked postsynaptic currents (ePSCs) the pipettes were filled with INLOW solution (Table 2-3). 
Table 2-1. Extracellular solution

\begin{tabular}{|l|c|l|}
\hline Substance & Concentration [mM] & Purchased from \\
\hline $\mathrm{NaCl}$ & 118 & Roth \\
\hline $\mathrm{NaHCO}_{3}$ & 25 & Roth \\
\hline $\mathrm{NaH}_{2} \mathrm{PO}_{4}$ & 1 & Roth \\
\hline $\mathrm{MgCl}_{2}$ & 1 & Sigma-Aldrich \\
\hline $\mathrm{KCl}$ & 3 & Sigma-Aldrich \\
\hline $\mathrm{CaCl}$ & 1,5 & Roth \\
\hline Glucose & 5 & Roth \\
\hline
\end{tabular}

Table 2-2.Intracellular INK solution

\begin{tabular}{|l|c|l|}
\hline Substance & Concentration [mM] & Purchased from \\
\hline $\mathrm{KCl}$ & 140 & Sigma-Aldrich \\
\hline $\mathrm{CaCl}_{2} \times 2 \mathrm{H}_{2} \mathrm{O}$ & 1 & Sigma-Aldrich \\
\hline $\mathrm{EGTA}$ & 10 & Sigma-Aldrich \\
\hline $\mathrm{MgCl}_{2} \times 6 \mathrm{H}_{2} \mathrm{O}$ & 2 & Sigma-Aldrich \\
\hline $\mathrm{Na}_{3} \mathrm{GTP}$ & 0,5 & Sigma-Aldrich \\
\hline $\mathrm{Na}_{3} \mathrm{ATP}$ & 4 & Sigma-Aldrich \\
\hline $\mathrm{HEPES}$ & 10 & Sigma-Aldrich \\
\hline
\end{tabular}

Table 2-3.Intracellular INLOW solution

\begin{tabular}{|l|c|l|}
\hline Substance & Concentration [mM] & Purchased from \\
\hline K-Gluconat & 140 & Sigma-Aldrich \\
\hline $\mathrm{CaCl}_{2}$ & 1 & Sigma-Aldrich \\
\hline $\mathrm{EGTA}$ & 10 & Sigma-Aldrich \\
\hline $\mathrm{MgCl}_{2} \times 6 \mathrm{H}_{2} \mathrm{O}$ & 2 & Sigma-Aldrich \\
\hline $\mathrm{Na}_{3} \mathrm{GTP}$ & 0,5 & Sigma-Aldrich \\
\hline $\mathrm{Na}_{3} \mathrm{ATP}$ & 4 & Sigma-Aldrich \\
\hline $\mathrm{HEPES}$ & 10 & Sigma-Aldrich \\
\hline
\end{tabular}


Intracellular solutions mentioned above were adjusted to $\mathrm{pH} 7.2$ with osmolarity about 310 mOSM.

Table 2-4. Drugs used for experiments

\begin{tabular}{|l|c|l|}
\hline Substance & Concentration $[\mu \mathrm{M} \& \mathrm{mM}]$ & Purchased from \\
\hline CNQX & $10 \mu \mathrm{M}$ & Tocris \\
\hline DL-AP5 & $10 \mu \mathrm{M}$ & Tocris \\
\hline Strychnine & $1 \mu \mathrm{M}$ & Sigma Aldrich \\
\hline Bicuculline & $1 \mu \mathrm{M}$ & Sigma Aldrich \\
\hline TTX & $0,5 \mu \mathrm{M}$ & Alomone labs \\
\hline Muscimol & $5 \mathrm{mM}$ & Tocris \\
\hline Glutamate & $5 \mathrm{mM}$ & Sigma Aldrich \\
\hline Glycine & $5 \mathrm{mM}$ & Roth \\
\hline Sucrose & $5 \mathrm{mM}$ & Roth \\
\hline
\end{tabular}

\subsubsection{Electrophysiological recordings}

All electrophysiological recordings were done on acute brainstem slices containing $\mathrm{PBC}$ and NH (Fig. 2.1). The slices were placed into the glass bottomed recording chamber and fixed by platinum wire with a grid of parallel nylon threads, to avoid of slice dislocation. The slice was continuously perfused with aerated extracellular solution during experiment, using a pump (Watson Marlow, 505LA ). The brain slices were visualised under an Axioscope microscope (Zeiss, Germany) using a $5 x$ objective. Somas of PBC neurons were identified under infraired gradient contrast illumination (C2400, Hamamatsu Photonics Deutschland $\mathrm{CmbH}$, Herrsching, Germany) with a 40x water immersion objective. Patch pipettes were pulled from borosilicate glass micropipettes (GC 150-10F, Clark Electromedical Instruments, UK) using a multistage puller (P87, Sutter Instrument Co., Novato, USA). When filled, they displayed resistance of 2-4 MS. Recordings were 
performed using an Axopatch 200 amplifier (Axon instrument Inc., USA). In experiments was used voltage-clamp configuration of the "whole-cell patch-clamp" technique. The first step in this method is formation of a gigaseal, by touching the cell surface with pipette and applying gentle suction. After application of a short pulse of negative pressure to the electrode the patch of membrane under the pipette is ruptured, reaching the whole-cell configuration. The solution filling the pipette enters into the cell and equilibrates with the cell's cytoplasm. After establishing of whole-cell configuration the recordings were performed at a holding potential of $-70 \mathrm{mV}$.

The capacitance and series resistance was compensated $80 \%$ according to manufacture recommendation. The membrane currents were filtered by a four-pole Bessel filter set at a corner frequency of $1 \mathrm{kHz}$ and digitized at a sampling rate of 5 $\mathrm{kHz}$ using the DigiData 1200 interface (Axon Instrument Inc., USA). For correction of current measurements $\mathrm{P} / 4$ protocol was used. According to this protocol four leak-subtraction pulses were applied immediately before the main command step and leak currents were subtracted. All experiments were conducted at $33-35^{\circ} \mathrm{C}$.

\subsubsection{Capacitance and series resistance calculation}

The passive properties of PBC neurons were estimated by determining membrane capacitance and series resistance for each recorded neuron before subsequent compensation. Capacitance and series resistance were calculated from the integral of the current transients induced by $20 \mathrm{mV}$ hyperpolorarizing voltage commands from a holding potential of $-70 \mathrm{mV}$ immediately after rupture of the cell membrane according to these formulas,

\section{$C=I \tau / 20$}

\section{$R s=\tau / C$}

where $C$ is the capacitance, $I$ is the current, $\tau$ is the decay, Rs is the series resistance. There were no systematic differences of capacitance and series resistance between different genotypes. 


\subsubsection{Recordings of spontaneous and evoked postsynaptic currents}

Evoked inhibitory and excitatory postsynaptic currents were recorded from hypoglossal motor neurons in presence of $10 \mu \mathrm{M}$ CNQX, DL-AP5 or $1 \mu \mathrm{M}$ bicuculline, $1 \mu \mathrm{M}$ strychnine, respectively. PSCs were evoked by $0.1 \mathrm{~Hz}$ field stimulations of axons near to preBötzinger complex using bipolar platinum stimulating electrode (30 $\mu \mathrm{M}$ diameter, Degussa, Germany). The pipettes were filled with INLOW solution (Table 2-3). For each experiment was applied supramaximal stimulation strength, using an isolation unit IsoFlex (A.M.P.I.) with a custom-built power supply. Peak amplitudes were averaged from 25 consecutive responses. The failure rate was calculated from these 25 responses in each experiment. To monitor changes in input resistance, current responses to a $-10 \mathrm{mV}$ voltage steps $(20 \mathrm{~ms})$ from a holding potential of $-70 \mathrm{mV}$ were recorded before every fifth stimulus. In all experiments the distance between stimulation and recording electrodes was similar on all slices from different mice. Spontaneous inhibitory and excitatory postsynaptic currents were recorded from neurons of preBötzinger complex at about $0 \mathrm{mV}$ in presence of $10 \mu \mathrm{M} \mathrm{CNQX}$, DL-AP5 or $1 \mu \mathrm{M}$ bicuculline, $1 \mu \mathrm{M}$ strychnine, respectively. Spontaneous miniature inhibitory and excitatory PSCs were recorded as described above, but adding $0.5 \mu \mathrm{M}$ tetrodoxin (TTX) into bath solution. For these experiments as a pipette solution was used INK intracellular solution (Table 2-2). Signals with amplitudes of at least two times above background noise were selected and statistical significance was tested in each experiment. In all experiments, as mentioned above, was used bath application of drugs, but in some experiments drugs were applied directly in close vicinity to the patched neurons, using a glass pipettes filled with muscimol $(5 \mathrm{mM})$, glycine $(5 \mathrm{mM})$, glutamate $(5 \mathrm{mM})$, or sucrose $(300 \mathrm{mM})$. Patches with a series resistance of $>20 \mathrm{M} \Omega$, a membrane resistance of $0.8 \mathrm{G} \Omega$ or leak currents of $>150 \mathrm{pA}$ were excluded. 


\subsubsection{Ventilation recordings}

Ventilation patterns were recorded by whole-body plethysmography. Unanaesthetized mice postnatal day 1 (P1) to P3 were placed in a closed chamber connected to a differential pressure transducer (CD15 Carrier Demodulator, ValiDyne). The analog signal of ventilation-related changes of air pressure was amplified and digitized using an A/D-converter (DigiData 3200, Axon Instruments). The coefficient of variation (CV) fv ventilation was calculated according to this formula,

\section{$\mathrm{CV}=\mathrm{Sd} / \mathrm{X}$}

where $\mathrm{Sd}$ is the standard deviation, $\mathrm{X}$ is the mean of the frequency.

\subsubsection{Data analysis}

Data acquisition and analyses was done using commercially available software: pClamp 6.0 and Axograph 4.6 (Axon Instruments Inc., USA), MiniAnalysis (SynaptoSoft, Decatur, GA), Prism 4 (GraphPad Software, San Diego, CA). All results are reported as mean \pm SEM. The statistical significance was evaluated with two-tailed unpaired Student's $t$ test.

\subsection{Fluorescence immunohistochemistry}

\subsubsection{Brain tissue preparation for immunohystochemsitry}

Postnatal NL2 KO mice (P5) were deeply anaesthetized with TBE (tribromoethanol) until they were unresponsive to painful stimuli. A thoracotomy was perormed and animals were perfused through the aorta with $0.9 \%$ sodium chloride followed by $100 \mathrm{ml} 2 \%$ paraformaldehyde in $0.1 \mathrm{M}$ phosphate buffer. The whole brain was removed, post-fixed for 1 hour in the same fixative at $4{ }^{\circ} \mathrm{C}$. The tissue was cryoprotected in $10-30 \%$ sucrose overnight at $4{ }^{\circ} \mathrm{C}$. Afterwards it 
was frozen by tissue freezing medium on dry ice and stored at $-80^{\circ} \mathrm{C}$. Series of transverse sections of brainstem with a thickness of $12 \mu \mathrm{m}$ were cut using a cryoslicer. Each section was quickly placed on the slide. After sectioning the slides were kept at $-80^{\circ} \mathrm{C}$.

\subsubsection{Solutions and chemicals}

Table 2-5 Chemicals used for immunohysochemistry

\begin{tabular}{|l|l|}
\hline Substance & Purchased from \\
\hline $\mathrm{NaCl}$ & Roth \\
\hline $\mathrm{KCl}$ & Sigma Aldrich \\
\hline $\mathrm{NaH}_{2} \mathrm{PO}_{4} \times \mathrm{H}_{2} \mathrm{O}$ & Roth \\
\hline $\mathrm{Na}_{2} \mathrm{HPO}_{4 \times} 12 \mathrm{H}_{2} \mathrm{O}$ & Roth \\
\hline Triton $\mathrm{X}-100$ & Sigma Aldrich \\
\hline $\mathrm{NGS}$ & Sigma Aldrich \\
\hline Mowiol & Calbiochem \\
\hline PFA & Roth \\
\hline TBE & Sigma Aldrich \\
\hline
\end{tabular}

\section{PFA $8 \%$ (for $1 \mathrm{~L}$ )}

$80 \mathrm{~g}$ paraformaldehyde in $0.1 \mathrm{mM} \mathrm{PB}$

$\underline{P B}$ (phosphate buffer for $2 L$ )

Buffer 1: $0.2 \mathrm{M} \mathrm{NaH}_{2} \mathrm{PO}_{4}\left(27,6 \mathrm{~g} \mathrm{NaH} 2 \mathrm{PO} 4 \mathrm{xH}_{2} \mathrm{O}\right)$

Buffer 2: $0.2 \mathrm{M} \mathrm{Na}_{2} \mathrm{HPO}_{4}\left(71.7 \mathrm{~g} \mathrm{Na}_{2} \mathrm{HPO}_{4 \times} 12 \mathrm{H}_{2} \mathrm{O}\right)$

\section{PBS (for $1 L)$}

$10 \mathrm{mM}$ PB; $150 \mathrm{mM} \mathrm{NaCl}$ 2,7mM KCl,

(50 ml PB; 8,77 g NaCl; 200 mg KCl; 900 ml H2O) 


\subsubsection{Antibodies used for immunofluorescence labeling}

Primary antibodies

Gephyrin, mouse

ProSAP, rabbit

VGlut 1, guinea pig

VGlut 2, guinea pig

VIAAT, rabbit
Synaptic system

Kindly provided by Dr. TM Boecker

Chemicon

Chemicon

Chemicon

Secondary antibodies

Goat Anti-GP Alexa Fluor 488

Goat Anti-mouse Alexa Fluor 555

Goat Anti-rabbit Alexa Fluor 488

Goat Anti-rabbit Alexa Fluor 555
Molecular probes

Molecular probes

Molecular probes

Molecular probes

\subsubsection{Immunofluorescence staining}

Before starting the immunostaining, the slices were washed three times with PBS. The blocking of non-specific binding sites and permeabilisiation of slices were done using $2 \%$ NGS and $0.2-0.3 \%$ Triton X-100 in phosphate-saline buffer (PBS) for 20-30 min at RT. Sections were incubated overnight at $4^{\circ} \mathrm{C}$ in primary antibodies dissolved in PBS containing $2 \%$ NGS and 0.2-0.3 \% Triton X100. After incubation with primary antibodies the sections were washed 3 times for each $10 \mathrm{~min}$ and then incubated for 1 Hour at RT in the dark with speciesspecific flurochrome-conjugated secondary antibodies, followed by three washing steps for 10 min each. Finally, sections were slightly air-dried and coverslipped with fluorescent mounting medium. 


\subsubsection{Data analysis}

Sections from immunofluorescence staining were visualized by confocal laser scanning microscopy (Zeiss LSM510). Typically, stacks of 7-12 images (1024x1024 pixel) at a zoom factor 4 spaced by $0.38-0.42 \mu \mathrm{m}$ were recorded, using a $63 x$ oil-immersion objective. For quantitative analyses, the gain and offset were held constant across all images to allow for intensity comparisons. Images were then imported into the AnalySIS software (Soft-Imaging Systems) and puncta were quantified. For quantifications, thresholds were manually determined for each image prior to binarization, followed by a particle separation filter. The resulting image was added to the original, and particle detection was carried out to measure particle number, defined area, mean intensity, and integral intensity. Data analysis was done using commercially available software (Prism 4 Software, Graph Pad).

\subsection{Biochemical methods}

\subsubsection{Solutions, antibodies used for biochemical procedures}

Homogenization buffer

$320 \mathrm{mM}$ sucrose; $5 \mathrm{mM}$ Hepes- $\mathrm{NaOH}(\mathrm{pH} 7.4) ; 0.1 \mathrm{mM}$ EDTA; proteases inhibitors (aprotinin, leupeptin)

Stacking gel buffer (Upper Tris)

O.5 M tris (pH 6.8); 0.4\% SDS

Separation gel buffer (Lower Tris)

$1.5 \mathrm{M}$ Tris (pH 8.8); 0.4\% SDS

10x SDS-PAGE-running buffer (for $1 \mathrm{~L}$ )

30.2 g Tris- $\mathrm{HCl}$; 144g Glycine; $10 \mathrm{~g}$ SDS 
10x Transfer buffer (for $1 \mathrm{~L}$ )

$31.2 \mathrm{~g}$ Tris- $\mathrm{HCl} ; 144 \mathrm{~g}$ Glycine; $5 \mathrm{~g}$ SDS

1x Transfer buffer (for $100 \mathrm{ml}$ )

$10 \mathrm{ml}$ 10x Transfer buffer; $20 \mathrm{ml}$ methanol; $70 \mathrm{ml} \mathrm{H}_{2} \mathrm{O}$

Ponseau-S solution (for $1 \mathrm{I}$ ):

2 g Ponceau-S; 50 ml Acetic acid; 950 ml $\mathrm{H}_{2} \mathrm{O}$

$10 \times$ TBS buffer:

$1.37 \mathrm{M} \mathrm{NaCl} ; 200 \mathrm{mM}$ Tris-HCl (pH 7.6)

1x TBS -Tween 20 buffer (for $1 \mathrm{~L}$ )

$100 \mathrm{ml} \mathrm{TBS} ; 900$ ml H $\mathrm{H}_{2}$; 1 ml Tween 20

Blocking buffer:

$5 \%$ non-fat powder milk in TBS-Tween 20 


\subsubsection{Primary and secondary antibodies}

Primary antibodies

$\mathrm{GABA}_{A} \mathrm{R} \alpha 1$, guinea pig

$\mathrm{GABA}_{A} R$ B2/3, mouse

$\mathrm{GABA}_{A} R$ g2, guinea pig

Gephyrin (3B11), mouse

GluR1, rabbit

GluR2/3, rabbit

$\mathrm{NL} 2$, rabbit

NMDAR1 (54.3), mouse

PSD-95, mouse

Synaptophysin (7.2), mouse

VGluT 1, guinea pig

VGluT 2, guinea pig

VIAAT, rabbit

Tubulin, mouse
Kindly provided by Dr. JM Fritschy

Chemicon

Kindly provided by Dr. JM Fritschy

Synaptic system

Chemicon

Chemicon

Kindly provided by Dr. F. Varoqueaux

Pharmingen

Abcam

Synaptic system

Chemicon

Chemicon

Chemicon

Sigma

Secondary antibodies

Goat Anti-GP HRP conjugated

Goat Anti-mouse HRP conjugated

Goat Anti-rabbit HRP conjugated
Dianova

Dianova

Acis antibodies

\subsubsection{Protein extracts preparation}

Brains from NL $2 \mathrm{KO}$ mice were quickly removed from the cut-open skull, brainstem was cut and immediately frozen by immersion in liquid nitrogen and stored at $-80^{\circ} \mathrm{C}$. The tissues of selected genotypes were homogenized in homogenization buffer with a glass Teflon homogenisator (homgen ${ }^{\text {plus }}$, Schütt) at 
setting $1200 \mathrm{rpm}$. Afterwards the homogenate was aliquoted and stored at $-20^{\circ} \mathrm{C}$. The protein concentration was measured with the Lowry assay (see in section 2.3.1). For using the protein sample was resuspended in $3 x$ loading buffer (see in section 2.3.1) and boiled at $100^{\circ} \mathrm{C}$ for $5 \mathrm{~min}$. The boiling denatures the proteins, unfolding them completely.

\subsubsection{Protein concentration estimation}

The total protein concentration was determined according to Lowry assay, using the total protein kit from Sigma with bovine albumin serum (Sigma-Aldrich, st. Louis $\mathrm{MO}$ ) as a standard. At first a BSA standard curve with sample of known protein concentration was prepared. A series of dilutions $(0,25,50,100,200$, $300,400 \mu \mathrm{g} / \mathrm{ml}$ ) were made in replicates of three with a final volume of $50 \mu \mathrm{l}$. Protein samples (1-3 $\mu \mathrm{l})$ were diluted in SDS to $50 \mu \mathrm{l}$. Each protein concentration measurement was performed in triplicate. The following step was the transferring of standards and samples into microplate (96 well plate; Sarsdedt Newton Inc., Newton USA). The Lowry reagent ( $50 \mu \mathrm{l})$ was added to each well and incubated for $20 \mathrm{~min}$ at RT, which was followed by addition of $25 \mu \mathrm{l}$ of Folin-Ciocalteu's phenol reagent. The addition of this reagent leads finally to an intensive blue staining, which was measured by absorbance at a wavelength between 500 and $800 \mathrm{~nm}$. All absorbance estimations were done using a Microplate reader (BioRad). Samples without protein were served as a reference. Analyses and statistics of the standard curve were performed using Sigma Plot software.

\subsubsection{SDS-polyacrilamide gel electrophoresis (SDS-PAGE)}

SDS-PAGE is a common biochemical method for protein separation. According to this method, proteins can be separated based on their molecular weight, as they move through polyacrilamide gel in response to an electric field. Protein samples before being subjected to electrophoresis were mixed with buffer containing SDS and ß-mercaptoethanol. SDS mediates the disruption of threedimensional structure of proteins by breaking non-covalent bonds and the loading 
of proteins with negative charges. ß-mercaptoethanol breaks disulfide bonds. Protein gel electrophoresis was performed using a minigel vertical apparatus. Glass-plate sandwich was built for preparation of the gel. The glass walls (10.5 $\mathrm{x}$ 10) were cleaned, sealed with silicone rubber band and clamped. After assembling the glass-plate sandwich of the electrophoresis apparatus, the stacking and separating gel solutions were prepared.

Table 2-7

\begin{tabular}{|l|c|c|}
\hline Solution & $\begin{array}{c}\text { Separation gel } \\
(10 \%)\end{array}$ & Stacking gel \\
\hline AA30 & $2.5 \mathrm{ml})$ \\
\hline Tris buffer $\mathrm{pH} 8.8$ & $1.875 \mathrm{ml}$ & $0.325 \mathrm{ml}$ \\
\hline Tris buffer $\mathrm{pH} 6.8$ & & $0.787 \mathrm{ml}$ \\
\hline $\mathrm{dH}_{2} 0$ & $3.125 \mathrm{ml}$ & $1.525 \mathrm{ml}$ \\
\hline Themed & $7.5 \mu \mathrm{l}$ & $3.7 \mu \mathrm{l}$ \\
\hline APS & $40 \mu \mathrm{l}$ & $20 \mu \mathrm{l}$ \\
\hline
\end{tabular}

The separation gel was poured first and the surface was covered with isopropanol, which straightens the surface of the gel. After the gel polymerized (in 30-40 min), isopropanol was removed. The stacking gel was then poured over the separation gel, and the comb of $0.6 \mathrm{~mm}$ thickness was inserted. When the stacking gel was polymerized (in 15-20 min), the comb and the silicone rubber band were removed, and the gel was then placed into the gel electrophoresis apparatus and filled with running buffer. Shortly before loading, the samples were boiled. The molecular weight marker (for estimating the molecular weight of unknown proteins) and samples were loaded into the wells of stacking gel using a Hamilton Syringe (Hamilton Company; Reno, Nevada, USA). Power supply was attached and the gel electrophoresis was carried out at 80 Volt until the samples got stacked at the lower border of the stacking gel, and then at 150 Volt for 1-2 Hours. 


\subsubsection{Western blotting}

The protein samples separated from SDS-PAGE can be transferred onto nitrocellulose membranes (Hybond ECL, Amersham), on the surface of which they are accessible to detection with specific poly- or monoclonal antibodies. Transfer of proteins from the gel onto nitrocellulose membrane was done using semi-dry blotting method. The transfer, nitrocellulose membrane and 6 sheets of Whatman filter papers of the same size as a gel were soaked with the transfer buffer for $15 \mathrm{~min}$. The transfer stack was assembled from the anode to the cathode in the following order: 3 sheets of Whatman filter paper, transfer membrane, gel and 3 sheets of Whatman filter paper and during this procedure bubbles were removed. For protein transfer a constant current of $150 \mathrm{~mA}$ was applied overnight. Afterwards the blotted membrane was removed and stained with removable Ponceau-S stain for 2 min at RT in order to test the efficiency of protein transfer. It was then distained by washing few minutes in TBS-Tween. In order to inhibit non-specific binding sites of antibodies to proteins, the membrane was first incubated in blocking solution for 1 hour at RT. Afterwards the membrane was incubated with the primary antibody in appropriate dilution in the blocking solution overnight at $4^{\circ} \mathrm{C}$. Each blot was stained in parallel for a reference protein (tubulin). After three washing steps for 10 min each with TBSTween, the membrane was incubated with HRP-conjugated antibody solution for 1 hour at RT, which binds to the heavy chain of primary antibody, followed by extensive washing steps. HRP coupled to the secondary antibody reduces the hydrogen peroxide and the resulting oxygen oxidizes the luminal, which releases the light. To visualize antigen-antibody reaction, enzymatic chemiluminescence's detection reagents were used (AceGlow reagents; psqLab biotechnoligie $\mathrm{GmbH}$ ). The detection reagents were mixed according to manufacturer's protocol. Briefly, the mixture of Solution A and B (1:1) was poured over the membrane for $1 \mathrm{~min}$ at RT. The membrane was placed into dark chamber and the light was detected by a CCD camera, which captures the digital image of the western blot. 


\section{Results}

Neuron 51, 741-754, September 21, 2006 @2006 Elsevier Inc. DOl 10.1016/j.neuron.2006.09.003

\section{Neuroligins Determine Synapse Maturation and Function}

Frédérique Varoqueaux, ${ }^{1,8}$ Gayane Aramuni, ${ }^{2,8}$ Randi L. Rawson, ${ }^{1}$ Ralf Mohrmann, ${ }^{3,4}$ Markus Missler, $2,5,6$ Kurt Gottmann, ${ }^{1,3,7}$ Weiqi Zhang, ${ }^{2}$ Thomas C. Südhof, ${ }^{5, *}$ and Nils Brose ${ }^{1,5, *}$

'Department of Molecular Neurobiology and Center for the Molecular Physiology of the Brain Max Planck Institute of Experimental Medicine D-37075 Göttingen

Germany

${ }^{2}$ Center for Physiology and Pathophysiology and Center for the Molecular Physiology of the Brain

Georg August University Göttingen

D-37073 Göttingen

Germany

${ }^{3}$ Department of Cell Physiology ND4

Ruhr University Bochum

D-44780 Bochum

Germany

${ }^{4}$ Department of Membrane Biophysics

Max Planck Institute of Biophysical Chemistry

D-37077 Göttingen

Germany

${ }^{5}$ Center for Basic Neuroscience

Department of Molecular Genetics

Howard Hughes Medical Institute

University of Texas Southwestern Medical Center

Dallas, Texas 75390

${ }^{8}$ Department of Genetics and Molecular Neurobiology

Otto von Guericke University Magdeburg

D-39106 Magdeburg

Germany

${ }^{7}$ Institute of Neurophysiology and Sensory Physiology

Heinrich Heine University Düsseldorf

D-40225 Düsseldorf

Germany

\section{Summary}

Synaptogenesis, the generation and maturation of functional synapses between nerve cells, is an essential step in the development of neuronal networks in the brain. It is thought to be triggered by members of the neuroligin family of postsynaptic cell adhesion proteins, which may form transsynaptic contacts with presynaptic $\alpha$-and $\beta$-neurexins and have been im plicated in the etiology of autism. We show that deletion mutant mice lacking neuroligin expression die shortly after birth due to respiratory failure. This respiratory failure is a consequence of reduced GABAergic glycinergic and glutamatergic synaptic transmission and network activity in brainstem centers that contro respiration. However, the density of synaptic contacts is not altered in neuroligin-deficient brains and cultured neurons. Our data show that neuroligins are required for proper synapse maturation and brain func

"Correspondence: thomas.sudhotelutsouthwestem.edu (T.C.S.) brose@em.mpg.de (N.B.)

${ }^{8}$ These authors contributed equally to this work. tion, but not for the initial formation of synaptic contacts.

Introduction

Synapses are asymmetric intercellular contact sites specialized for temporally and spatially precise signal transmission in the brain. They are composed of a presynaptic compartment containing synaptic vesicles clustered around the transmitter release site, or active zone, and a postsynaptic compartment containing the transmitter reception apparatus. During synaptogenesis in human brain development, about $10^{11}$ nerve cells generate some $10^{15}$ synapses to establish a complex but precise neuronal network.

Synaptogenesis involves two operationally defined and interdependent cell biological processes that are thought to be mediated by adhesion proteins (Serafini, 1999; Yamagata et al., 2003), the initial contact formation between an axonal growth cone and a target neuron, and the maturation of synaptic contacts through specific assembly and stabilization of pre- and postsynaptic proteins at the contact site. Adhesion proteins involved in the initial formation of synaptic contacts are thought to be encoded by large families of genes and to interact with each other in an isoform-specific manner according to a "lock-and-key" principle in order to allow for the cell-type specificity of synaptogenesis (Sperry, 1963) Adhesion proteins that mediate protein recruitment during synaptogenesis, on the other hand, additionally require specific intracellular binding sites for scaffolding proteins, presynaptic active zone components, and postsynaptic receptors (Gamer et al., 2002; Kim and Sheng, 2004; $\mathrm{Li}$ and Sheng, 2003; Montgomery et al., 2004; Scheiffele, 2003; Yamagata et al., 2003).

Several recent studies on the molecular mechanism of synaptogenesis indicated a role of neuroligins (NLs) in the formation of synaptic contacts and their maturation (Chih et al., 2005; Chubykin et al., 2005; Dean et al., 2003; Fu et al., 2003; Graf et al., 2004; Levinson et al., 2005; Prange et al., 2004; Scheiffele et al., 2000). NLs are type 1 transmembrane proteins and constitute a family of four (in rodents) or five (in higher primates and humans) postsynaptic cell adhesion proteins that interact with presynaptic $\alpha$ - and $\beta$-neurexins (NXs) (Boucard et al., 2005; Ichtchenko et al., 1995; Song et al., 1999; Varoqueaux et al., 2004) via a large extracellular esterase-like domain. Intracellularly, NLs bind to several PDZ domain-containing scaffolding proteins, which in tum interact with postsynaptic transmitter receptors, ion channels, and signaling proteins (Hirao et al., 1998; Irie et al., 1997; Meyer et al., 2004). Similarly, the intracellular domains of NXs, which are also type 1 transmembrane proteins, are indirectly associated with components of the presynaptic transmitter release machinery via interactions with PDZ domain-containing scaffolding proteins (Biederer and Südhof, 2000; Hata et al., 1996).

Expression of NLs in nonneuronal cells induces presynaptic specializations in contacting axons (Scheiffele et al., 2000), and overexpression of NLs in cultured 
neurons increases the number of synapses formed (Chih et al., 2005; Dean et al., 2003; Graf et al., 2004; Levinson et al., 2005; Nam and Chen, 2005; Prange et al., 2004), whereas knock-down of NL expression by RNAi leads to a reduction in synapse density (Chih et al., 2005). Analogous effects were observed upon expression of $\beta-N X s$ in nonneuronal cells (Graf et al., 2004; Nam and Chen, 2005). Overexpression and knock-down of NLs in cultured neurons causes dramatic changes in synaptic activity, which were explained by the dramatic effects of these manipulations on synapse numbers (Chih et al., 2005; Graf et al., 2004). Accordingly, the transsynaptic NUNX link is thought to be of key importance in the induction phase of synaptogenesis. NLs are thought to trigger synapse formation. In addition, NLs were suggested to also play a role in synapse maturation by recruiting scaffolding proteins, postsynaptic receptors, and signaling proteins to nascent synapses. That NLs are essential for proper brain function is documented by the fact that loss-of-function mutations in the human NL genes NLGN3 and NLGN4 cause autism and mental retardation (Chih et al., 2004; Comoletti et al., 2004; Jamain et al., 2003; Laumonnier et al., 2004; Talebizadeh et al., 2006).

To determine the role of NLs in synaptogenesis and synapse function in vivo, we generated and characterized knockout $(\mathrm{KO})$ mice lacking individual or multiple NLs.

Results

Expression of NL Genes in Rodents

The mouse genome contains at least four NL genes: Nlgn1, Nign2, Nlgn3, and Nlgn4 (Ichtchenko et al., 1996; S. Jamain, F.V., N.B, and T. Bourgeron, unpublished data). We employed quantitative Westem blotting with isoform-specific antibodies to determine the absolute levels of the four NLs in the brains of newborn and adult mice. In newbom mice, NL 4 is not detectable (Table 1) whereas NLs 1-3 are expressed at moderate levels (NL 1, $65 \mathrm{pg} / \mathrm{mg} ; \mathrm{NL} 2,65 \mathrm{pg} / \mathrm{mg} ; \mathrm{NL} \mathrm{3,82} \mathrm{pg/mg).} \mathrm{In}$ adult mice, only $3 \%(14 \mathrm{pg} / \mathrm{mg})$ of the total NL protein in brain are contributed by NL 4, while NL 1-3 are expressed at 2- to 3-fold higher levels than in newbom brain (NL 1, 113 pg/mg; NL 2, 170 pg/mg; NL 3, 222 $\mathrm{pg} / \mathrm{mg}$ ). In view of these low levels of $\mathrm{NL} \mathrm{4}$, we focused all subsequent studies on the characteristics of NLs 1-3 in mouse brain.

The mRNAs of NLs 1-3 are expressed at low levels in newborn rat brain and upregulated in parallel (2- to 3 fold) during postnatal development (Figure 1A), reflecting the developmental expression patterns seen for NL protein levels in mouse and rat brain, as described above and in previous studies (Song et al., 1999; Varoqueaux et al., 2004). In situ hybridization experiments showed that in both the newbom and adult rat brain, the mRNAs of NLs 1-3 are coexpressed in almost all neuronal populations (Figures $1 \mathrm{~B}$ and $1 \mathrm{C}$ ). Differential expression of NL isoforms is only detectable in the newborn striatum and adult brainstem, hypothalamus, and thalamus, where relative NL 1 mRNA expression levels are lower than those of NL 2 and NL 3 mRNAs (Figures $1 \mathrm{~B} 1$ and $1 \mathrm{C})$. In agreement with our previous studies on NL mRNA and protein expression (Song et al.,

\begin{tabular}{|c|c|c|c|}
\hline $\begin{array}{l}\text { Presynaptic } \\
\text { Proteins }\end{array}$ & & $\begin{array}{l}\text { Postsynaptic } \\
\text { Proteins }\end{array}$ & \\
\hline complexin1 & $78 \pm 4$ & B-catenin ${ }^{(x)}$ & $102 \pm 3$ \\
\hline complexin2 & $70 \pm 2^{2 m+*}$ & $\mathrm{~N}$-cadherin & $112 \pm 3$ \\
\hline Munc13-1 & $108 \pm 12$ & B-dystroglycan & $117 \pm 15$ \\
\hline RIM1/2 & $95 \pm 12$ & gephyrin & $110 \pm 11$ \\
\hline ¿SNAP & $82 \pm 6^{*}$ & $\mathrm{KCC} 2$ & $80 \pm 5^{\circ *}$ \\
\hline SNAP-25 & $88 \pm 6$ & GlyRix & $97 \pm 3$ \\
\hline synaptobrevin 2 & $77 \pm 5^{*}$ & $\mathrm{GABA}_{\alpha} \mathrm{R} \alpha 1$ & $99 \pm 1$ \\
\hline synaptophysin 1 & $46 \pm 9^{* *}$ & $\mathrm{GABA}_{A} \mathrm{R} / 2 / 3$ & $97 \pm 4$ \\
\hline synaptotagmin 1 & $78 \pm 6^{*}$ & GluR2/3 & $83 \pm 4$ \\
\hline VIAAT ${ }^{[x]}$ & $76 \pm 9$ & NMDAR1 & $77 \pm 1^{* *}$ \\
\hline VGIUTI & $62 \pm 4^{* *}$ & NMDAR2A/B & $106 \pm 9$ \\
\hline VGluT $2^{(n)}$ & $57 \pm 2$ & PSD-95 & $91 \pm 4$ \\
\hline calbindin & $92 \pm 4$ & neuroligin 4 & not detectable \\
\hline
\end{tabular}

Protein levels in newborn brain homogenates, expressed as percent of wild type levels \pm SEM, are listed (wild type, $n=3 ;$ TKO, $n=8$; [a] control, $n=3 ;$ TKO, $n=4 ; " p<0.05, " p<<0.01, " \cdots p<0.001)$.

1999; Varoqueaux et al., 2004), areas rich in white matter exhibit only background labeling for NL mRNAs, indicating that oligodendrocytes and fibrous astrocytes express NL mRNAs either at extremely low levels or not at all (Figures $1 \mathrm{~B}$ and $1 \mathrm{C}$ ).

\section{Generation of NL KO Mice}

To generate mouse KOs lacking NLs 1-3, exon sequences covering the translational start site and at least $380 \mathrm{bp}$ of $5^{\prime}$ coding sequence of the respective genes were deleted by homologous recombination in embryonic stem cells (see Figures S1A and S1B in the Supplemental Data available online). In all cases, this targeting strategy eliminates the respective signal sequences and a significant part of the extracellular esterase-like domain and was therefore predicted to abolish NL expression completely. This was verified by Western blotting of brain homogenates from homozygous KOs using antibodies to the C termini of NLs 1-3, which showed that neither full-length NLs (Figure S1C) nor truncated variants (data not shown) were expressed in the respective $\mathrm{KO}$ mice.

KOs lacking individual NLs and all combinations of double $\mathrm{KO}$ s were generated by interbreeding and were obtained at the predicted Mendelian frequencies. All single $\mathrm{KOs}$, all types of double heterozygous mice, and triple heterozygous mice were viable and fertile and behaved normally in the cage environment. Likewise, all homozygous double $\mathrm{KO}$ combinations were viable. However, they showed drastically reduced reproduction rates and striking deficits in raising their offspring. As a consequence, breeding of triple KOs lacking NLs 1-3 was extremely time consuming, even with very large breeding colonies. Nevertheless, triple KOs were obtained at the predicted Mendelian frequency. They had a slightly reduced body weight as compared to single and double KO littermates (Figure 2A), showed irregular and flat breathing movements as determined by wholebody plethysmography (Figures $2 \mathrm{~B}$ and $2 \mathrm{C}$ ), and died within $24 \mathrm{hr}$ after birth. NL 4 levels were not upregulated in triple $\mathrm{KO}$ brains (Table 1).

Currently, subcellular localization data on NL proteins in the brain are only available for NL 1 and NL 2 . In the 
A

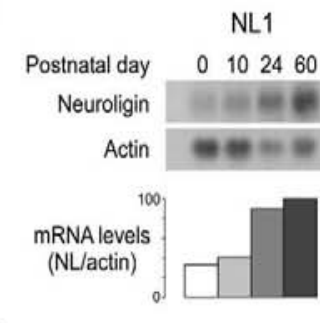

B1

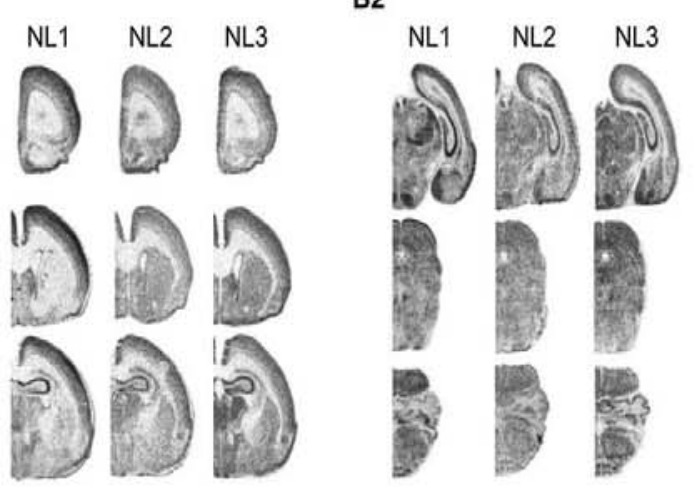

C

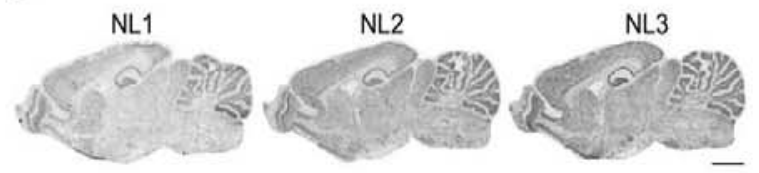

Figure 1. Expression Profiles of NL1, NL2, and NL3 mRNAs during the Synaptogenesis Phase of Rat Brain Development

(A) Northern blot analysis of NL and actin mRNA levels in postnatal brain homogenates at postnatal days $0,10,24$, and 60 . All three NL mRNAs are concomitantly expressed and upregulated during the main synaptogenesis phase of brain development (postnatal days 0 -24).

(B1, B2, and C) At all times of postnatal development, NL1, NL2, and NL3 mPNAs are ubiquitously expressed throughout the brain, as shown by in situ hybridization in the newborn (B1 and $B 2$ ) and the adult rat brain (C). Scale bar, $1.6 \mathrm{~mm}(\mathrm{~B}), 3.4 \mathrm{~mm}$ (C). rodent brain, the main NL 1 splice variant is specifically localized to excitatory postsynaptic densities (Song et al., 1999; Chih et al., 2006), whereas the main NL 2 splice variant is specifically localized to inhibitory postsynaptic specializations (Graf et al., 2004; Varoqueaux et al., 2004; Chih et al., 2006). However, both NL 1 and NL 2 alter the density of excitatory and inhibitory synapses when overexpressed in cultured neurons (Graf et al., 2004; Levinson et al., 2005), some less abundant NL. 1 and NL 2 splice isoforms are targeted to and induce inhibitory and excitatory synapses, respectively (Chih et al., 2006), and knock-down of individual NLs by RNAi or dominant-negative NLs cause comparable reductions of synapse numbers in neuron cultures (Chih et al., 2005; Nam and Chen, 2005). Moreover, the three NLs present in newborn brain, NL 1-3, are coexpressed in almost all types of neurons (Figure 1B), and only the triple KO lacking NLs 1-3 but none of the single or double KOs showed a perinatally lethal phenotype. As all these findings indicate a significant degree of functional redundancy among NLs 1-3, we studied the phenotype of NL 1-3 triple KOs in more detail.

Brain Cytoarchitecture and Synaptic Protein Expression in NL 1-3 Triple KO Mice

Histological analyses showed that the loss of NL expression in triple KOs had no effect on the gross cytoarchitecture of the newbom brain. The density and layering of neurons in olfactory bulb, cortex, and hippocampus of triple KOs were indistinguishable from those in triple heterozygous controls (Figure S2). Even brain regions that are mature at birth, such as the brainstem, did not show evidence of cell loss (data not shown).

Analysis of total brain expression levels of selected synaptic marker proteins in triple KOs and wild-type controls by quantitative Western blotting revealed a complex pattem of changes (Table 1). The levels of all tested general, excitatory, and inhibitory synaptic vesicle markers were reduced in triple KO brains. Likewise, the levels of the soluble SNARE regulators complexin 2 and $\alpha$ SNAP were significantly lower in triple KOs as compared to controls, whereas the expression levels of the active zone proteins Munc13-1 and RIM1/2, the SNARE SNAP-25, and calbindin were unchanged. The $\mathrm{Cl}^{-}$channel KCC2 showed reduced expression levels in triple KO brains, while the expression of gephyrin, $\beta$-dystroglycan, GABA $_{A}$ receptor subunits $\alpha 1$ and $\beta 2 / 3$, and glycine receptor $\alpha$ subunits, all markers for GABAergic/glycinergic postsynapses, was normal. Upon analysis of markers of glutamatergic excitatory postsynapses, only the expression levels of NMDAR1 were found to be reduced significantly in triple KOs. The levels of PSD-95 and of the glutamate receptor subunits GluR2/3 and NMDAR2A/B were similar in triple $\mathrm{KO}$ and control brains. The finding that the levels of integral components of synaptic contacts, such as the active zone markers Munc13-1 and 
A

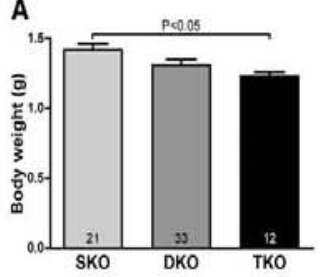

B
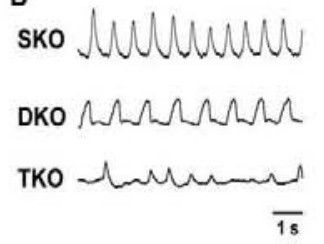

C

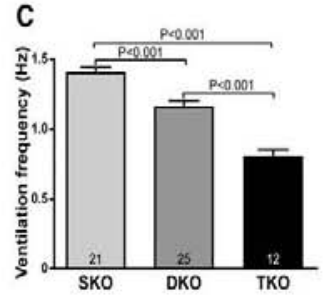

Figure 2. Essential Role of NLs in Respiratory Function

(A) Averaged body weight of NL single (SKO), double (DKO), and triple (TKO) KO mice. (B) Representative ventilation traces and (C) averaged ventilation frequencies of different NL mutant mice as measured by whole-body plethysmography. Numbers within the bar graphs indicate the number of mice tested for each genotype. Error bars indicate SEM.

RIM1/2, are not affected in the NL 1-3 triple KO whereas the expression levels of vesicle markers are reduced is compatible with the notion that loss of NLs results in aberrant recruitment of synaptic proteins or vesicles but does not cause changes in the number of synaptic contacts (see below).

Synapse Formation and Function in Cultured NL 1-3 Triple KO Neurons

One of the best characterized NL interaction partners is the postsynaptic density protein PSD-95. It binds to NLs via its third PDZ domain (Irie et al., 1997), as well as to $\mathrm{K}^{+}$ channels (Kim et al., 1995), NMDA receptor subunits (Komau et al., 1995), and other scaffolding and signaling proteins at its two other PDZ domains and additional binding sites (Garner et al., 2002; Li and Sheng, 2003; Montgomery et al., 2004; Scheiffele, 2003). PSD-95 also binds to stargazin, which in tum interacts with AMPA receptors (Fukata et al., 2005; Schnell et al., 2002). Thus, PSD-95 represents an adaptor between synaptic cell adhesion and the postsynaptic signaling apparatus. Experiments in neuron cultures showed that PSD-95 presumably through its interaction with NLs, and stargazin mediate the synaptic recruitment of glutamate receptors (El-Husseini et al., 2002; Fukata et al., 2005; Levinson et al., 2005; Prange et al., 2004; Schnell et al., 2002).

To examine the role of NLs in synaptogenesis and the regulation of the glutamate receptor complement at syn- apses, we studied glutamatergic synaptic transmission in cocultures of neocortical explants and dissociated neocortical target neurons from the same NL 1-3 triple KO mice by whole-cell patch-clamp recording at 13-15 DIV (Mohrmann et al., 1999). Cultures from triple heterozygous littermates served as controls and yielded data that were very similar to those obtained in preparations from an unrelated wild-type mouse strain (Figures S3C$\mathrm{S} 3 \mathrm{E}$ ). In this system, the frequency and amplitude of miniature excitatory postsynaptic currents (mEPSCs), the AMPA and NMDA receptor-mediated contributions to evoked excitatory postsynaptic currents (eEPSCs), and the specific loss of AMPA or NMDA receptors from postsynapses can be measured with high fidelity (Mohrmann et al., 1999) and can serve as readouts for the effects of NL deletion on synaptogenesis and synaptic receptor recruitment. Neither the frequency nor the amplitude of AMPA mEPSCs differed significantly between neurons obtained from triple $\mathrm{KO}$ s and heterozygous controls (Figures S3A, S3C, and S3D). These results are in line with recent electrophysiological data obtained in RNAi knock-down experiments on cultured neurons, where a strong reduction of NL 1-3 expression had little effect on AMPA mEPSC frequency or amplitude (Chih et al., 2005). In subsequent experiments, we examined the ratio of evoked NMDA receptor- and AMPA receptor-mediated eEPSCs at the same set of synapses. No significant differences in AMPA eEPSCs, NMDA eEPSCs, or their ratio were found between triple KO neurons and heterozygous control neurons in this culture preparation (Figures S3B and S3E). In contrast, NMDA receptor-mediated, but not AMPA receptormediated, synaptic transmission onto CA1 pyramidal cells appears to be perturbed in acute hippocampal slices from NL 1 KO animals (A.A. Chubykin, J.R. Gibson, D. Atasoy, N.B., E.T. Kavalali, and T.C.S, unpublished data).

The electrophysiological features of NL 1-3 triple KO cortical neurons agreed with the momhological characteristics of hippocampal and cortical neurons from these mutants. Immunofluorescence double staining of the synaptic markers synapsin and PSD-95 revealed no differences in synapse densities of neuron cultures from triple $\mathrm{KO}$ and control hippocampus (Figures $3 \mathrm{~A}$ and 3B). Moreover, the ultrastructure of synapses formed by NL 1-3 triple KO neurons was indistinguishable from the triple heterozygous control situation, as assessed by morphometric electron microscopic analyses of cortical explants at DIV 18 (Figures $3 \mathrm{C}$ and $3 \mathrm{D}$ ). These electrophysiological and morphological findings indicate that the initial formation of synaptic contacts does not depend on NLs.

\section{Respiratory Failure in NL 1-3 Triple KO Mice}

Because of the apparent synaptogenic effects of NL overexpression in cultured neurons (Chih et al., 2005; Dean et al., 2003; Graf et al., 2004; Levinson et al., 2005; Nam and Chen, 2005; Prange et al., 2004; Scheiffele et al., 2000) and the proposed role of NLs in the synaptic recruitment of glutamate receptors (Chih et al., 2005; Dean et al., 2003; Graf et al., 2004; Levinson et al., 2005; Nam and Chen, 2005; Prange et al., 2004; Scheiffele et al., 2000; Serafini, 1999) we had initially predicted that $\mathrm{KO}$ of all NLs in mouse brain neurons would 
A

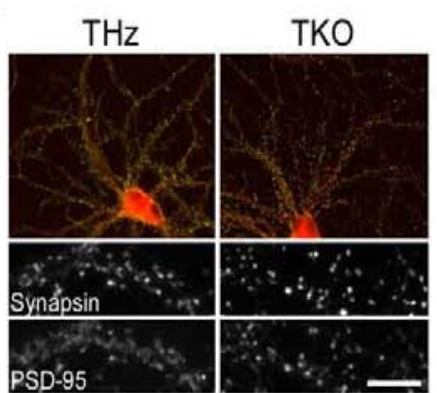

B

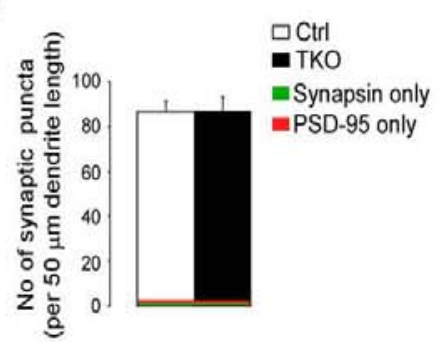

C

$\mathrm{THz}$

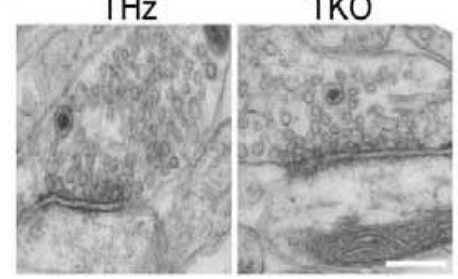

D

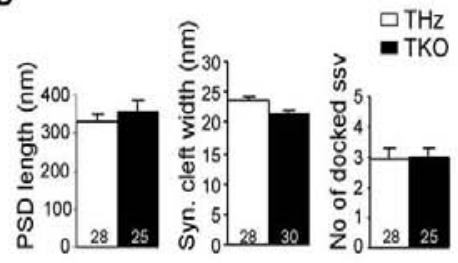

Figure 3. Normal Synaptogenesis and Synaptic Morphology in NL 1-3 Triple KO Hippocampal and Neocortical Neurons

(A) Combined immunofluorescence detection of synapsin (presynaptic marker) and PSD-95 (glutamatergic postsynaptic marker) in cultured hippocampal neurons, showing that synapses are formed at normal densities by the NL 1-3 triple KO (TKO) neurons, as compared to triple heterozygous controls $(\mathrm{THz})$.

(B) Quantification of synapsin-labeled puncta per dendrite length of control (ctrl) and NL1-3 triple KO (TKO) neurons. The vast majority $(>98 \%)$ of these puncta were found in close apposition to PSD-95 immunoreactive puncta.

(C and D) Cortical explants after 2 weeks in culture. Mature synaptic specializations (C) were observed on NLtriple KO (TKO) as well as on control $(\mathrm{THz})$ neurons, and synaptic parameters (length of the postsynaptic density [PSD], width of the synaptic clett, number of docked vesicles) were similar in both experimental groups (D).

Numbers within the bar graphs indicate the number of synapses studied. Scale bars, $20 \mu \mathrm{m}$ (A), $150 \mathrm{~nm}$ (C). Error bars indicate SEM. result in decreased synapse numbers and/or aberrant glutamatergic synaptic transmission, but our experimental approaches to test these predictions in cultured neurons failed to uncover phenotypic changes in NL triple KO neurons (Figures 3 and S3). A reason for this finding may be that phenotypic changes induced by NL deletion can be obscured in neuron culture preparations.

We therefore turned to the most obvious phenotypic characteristic of the triple KOs, their early postnatal death, which is most likely caused by respiratory failure, as NL 1-3 triple KOs show flat and irregular breathing movements (Figure 2). We examined the function of the brainstem respiratory network consisting of the pre-Bötzinger complex (PBC) and the hypoglossal nucleus $(\mathrm{NH})$ in acute brainstem slices from triple $\mathrm{KO}$ mice by whole-cell patch-clamp recordings (Missler et al., 2003; Zhang et al., 2005). Because this type of analysis required large numbers of triple $\mathrm{KO}$ animals and it is practically impossible to obtain sufficient numbers of NL 1-3 triple KOs and wild-type control litermates, we followed a breeding strategy using mice that were homozygous KOs for one NL allele (mostly NL 3, some NL 1, no NL 2) and heterozygous for the two others. As a consequence, single KO (NL 3 or NL 1, no NL 2) and double KO littermates (NL 1 and NL 3) had to serve as controls in these experiments. For statistical purposes, the datasets from these two control groups of animals were pooled separately and are referred to as single KO and double KO below. In all cases, the pooled data from single KOs approached wild-type control levels, which were measured in a wild-type line with a similar mosaic genetic background as the NL 13 triple KO (data not shown). Essentially, this statistical analysis underestimates phenotypic changes contributed by the KO of NL 1 or NL 3 .

We first recorded voltage-activated $\mathrm{Na}^{+}, \mathrm{K}^{+}$, and $\mathrm{Ca}^{2+}$ currents in the PBC, which is essential for generating a normal respiratory rhythm. Neither $\mathrm{Na}^{+}$nor $\mathrm{K}^{+}$or $\mathrm{Ca}^{2+}$ current densities were significantly altered in NL 1-3 triple KO neurons of the PBC (Figure S4). To test whether synaptic function is impaired in the brainstem of triple KOs, we next analyzed synaptic transmission in the PBC. Spontaneous postsynaptic currents (sPSCs) measured in whole-cell patch-clamp recordings from PBC neurons revealed a dramatically reduced frequency of total sPSCs as well as of pharmacologically separated GABAergic and glycinergic sPSCs in NL 1-3 triple KOs as compared to single KOs, while sPSC amplitudes were less affected (Figures 4A-4F). Different double KO combinations caused a similar but less pronounced phenotype. Analysis of the amplitude distribution of GABAergic and glycinergic sPSCs showed two distinct populations in single KOs with mean amplitudes of 35 and $100 \mathrm{pA}$, which most likely correspond to miniature and action potential driven events, respectively (Figure 4F). Deletion of two NLs decreased the highamplitude population of GABAergic and glycinergic sPSCs by more than $50 \%$, while the number of sPSCs with lower amplitude was only moderately changed $(<20 \%)$. In triple $\mathrm{KO}$, the number of GABAergic and glycinergic SPSCs with lower and higher amplitude were decreased by $85 \%$ and $95 \%$, respectively. In an additional series of experiments, we analyzed miniature 
A

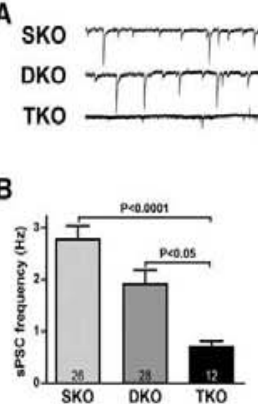

D

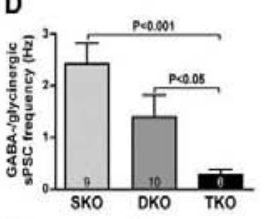

F

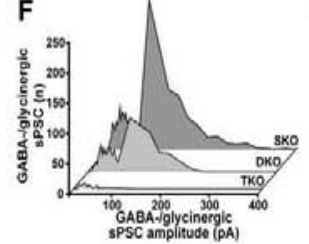

H
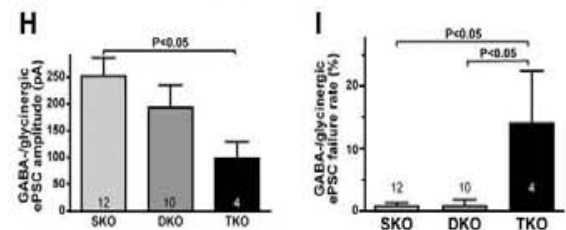

J

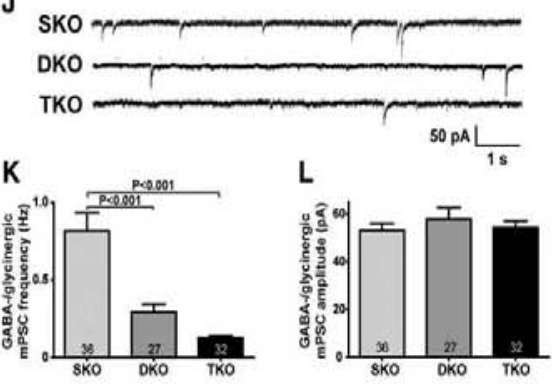

Figure 4. Impaired GABAergic/Glycinergic Neurotransmission in the Respiratory Centers of the NL. 1-3 Triple KO

(A) Representative recordings of phamacologically isolated spontaneous GABAergic/glycinergic postsynaptic currents (SPSC) in brainstem PBC neurons. Slices were prepared from littermate NL single (SKO), double (DKO), and triple KO (TKO) mice.

(B and C) Frequency (B) and amplitude (C) of total sPSCs in PBC neurons.

(D-F) Frequency (D), amplitude (E), and amplitude distribution (F) of pharmacologically isolated GABAergic/glycinergic SPSCs in PBC neurons.

( $(\mathrm{G}$ and $\mathrm{H}$ ) Impaired evoked GABAergic/glycinergic neurotransmission in hypoglossal neurons from NL KO mice. Sample traces of evoked GABAergic/glycinergic ePSCs (G) and the mean amplitudes of evoked GABAergic/glycinergic ePSCs in response to extracellular stimuli $(H)$ are given.
GABAergic and glycinergic PSCs (mPSCs) in PBC neurons directly. While the frequency of GABAergic/glycinergic mPSCs was reduced by $80 \%$ in triple $\mathrm{KO}$ cells as compared to single $\mathrm{KO}$ control cells, the amplitudes of GABAergic/glycinergic mPSCs were similar in all tested genotypes (Figures $4 \mathrm{~J}-4 \mathrm{~L}$ ).

To examine the effects of the NL 1-3 deletion on glutamatergic synaptic transmission in the respiratory brainstem, we monitored phamacologically isolated spontaneous glutamatergic postsynaptic currents in PBC neurons. In comparison to data from single KO slices, the frequency of glutamatergic sPSCs in PBC neurons was decreased in NL 1-3 triple KO slices (Figures 5A and $5 B$ ). The amplitudes of glutamatergic sPSCs were similar in all tested genotypes (Figures $5 \mathrm{C}$ and $5 \mathrm{E}$ ). In order to detect differential effects of the NL 1-3 triple $\mathrm{KO}$ on glutamatergic and GABAergic/glycinergic synaptic transmission, we determined the relative contribution of glutamatergic sPSCs and GABAergic/glycinergic sPSCs to the total synaptic events in a subset of experiments. In all single KOs, most events were GABAergic/ glycinergic sPSCs $(80 \%)$, and glutamatergic sPSCs contributed only $20 \%$ of the total synaptic activity (Figure $5 \mathrm{D}$ ). This ratio is very similar to that observed in respiratory brainstem slices from newborn wild-type mice (Missler et al., 2003). Deletion of two or three NLs dramatically changed the balance between glutamatergic and GABAergic/glycinergic SPSCs, with glutamatergic sPSCs contributing $70 \%$ and GABAergic/glycinergic sPSCs contributing $30 \%$ of all synaptic events in NL 1-3 triple KOs (Figure 5D). This change did not result from an increase of total glutamatergic activity, but was rather a consequence of the strong decrease in GABAergic/ glycinergic SPSC frequency. When we analyzed miniature glutamatergic mPSCs in PBC neurons directly, we observed an $80 \%$ reduction in mPSC frequency in triple KO cells as compared to single KO controls, whereas the amplitudes of glutamatergic mPSCs were similar in all tested genotypes (Figures $5 \mathrm{H}-5 \mathrm{~J}$ ).

Finally, we examined electrically evoked synaptic transmission in the NH. For this purpose, we stimulated neurons in the vicinity of the PBC and measured synaptic transmission to $\mathrm{NH}$ neurons by whole-cell patch-clamp recordings in the presence of CNQX for GABAergic/ glycinergic ePSCs, or bicuculline/strychnine for the analysis of glutamatergic ePSCs. Compared to slices from single KOs, the average amplitude of GABAergic/glycinergic ePSCs was strongly decreased in $\mathrm{NH}$ neurons from NL 1-3 triple KOs, whereas GABAergic/glycinergic ePSC amplitudes in double KO neurons were not changed significantly (Figures $4 G$ and $4 \mathrm{H}$ ). Despite the fact that we used supramaximal stimulation, the failure rate of GABAergic/glycinergic ePSCs was strongly increased in NL 1-3 triple KO neurons as compared to controls (Figure 4l). In contrast to GABAergic/glycinergic

(I) Failure rates of evoked GABAergic/glycinergic neurotransmission in hypoglossal neurons from NL KO mice.

(J) Representative recordings of pharmacologically isolated spontaneous miniature GABAergic/glycinergic mPSCs in PBC neurons. (K and L) Frequency (K) and amplitude (L) of GABAergic/glycinergic mPSCs in PBC neurons.

Numbers within or above the bar graphs indicate the number of mice tested for each genotype. Error bars indicate SEM. 
A

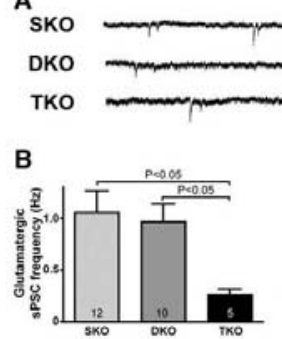

D

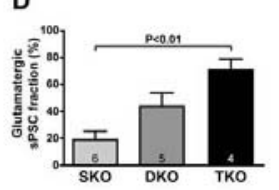

F

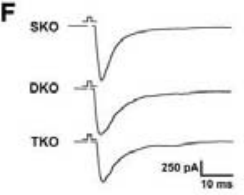

G

E
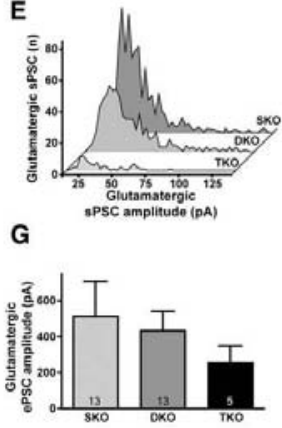

H

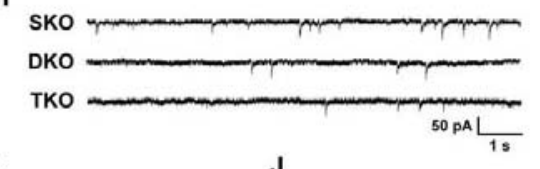

I

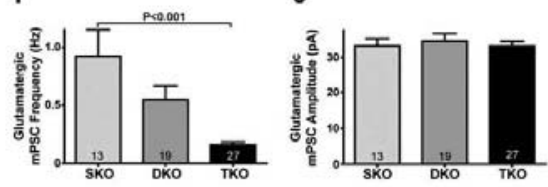

Figure 5. Impaired Glutamatergic Neurotransmission in the Respiratory Centers of the NL 1-3 Triple KO

(A) Representative recordings of pharmacologically isolated spontaneous glutamatergic postsynaptic currents (sPSC) in brainsten PBC neurons. Slices were prepared from littermate NL single (SKO), double (DKO), and triple KO (TKO) mice. (B and C) Frequency $(\mathrm{B})$ and amplitude $(\mathrm{C})$ of glutamatergic sPSCs in PBC neurons.

(D) CNQX-sensitive glutamatergic fraction of sPSCs, expressed as percent of total sPSCs in PBC neurons of NL KO mice.

(E) Amplitude distribution of spontaneous glutamatergic sPSCs in PBC neurons of NL KO mice.

(F and G) Reduced evoked excitatory neurotransmission in hypoF and G) Reduced evoked excitary neurotransmission in hypoglossa neurons the NL KO mice. Sample traces of evoked glutamatergic ePSCs $(F)$ and the mean amplitudes of evoked glutama gic ePSCs in response to extracellular stimuli (G) are given. (H) Representative recordings of pharmacologically isolated spon taneous glutamatergic mPSCs in PBC neurons.

$(\mathrm{I}$ and $\mathrm{J})$ Frequency $(\mathrm{l})$ and amplitude $(\mathrm{J})$ of glutamatergic mPSCs in PBC neurons.

Numbers within the bar graphs indicate the number of mice tested for each genotype. Error bars indicate SEM.
ePSCs, the amplitudes of glutamatergic ePSCs were not significantly different in $\mathrm{NH}$ neurons from single, double, or triple $\mathrm{KO}$ (Figures $5 \mathrm{~F}$ and $5 \mathrm{G}$ ), and failure rates were similar in all tested genotypes $(3.9 \% \pm 2.3 \%, n=13$, $5.0 \% \pm 3.5 \%, n=13$, and $4.0 \% \pm 1.2 \%, n=5$, for single, double, and triple KOs, respectively).

Synaptogenesis in the Brainstem of NL 1-3 Triple KO Mice

The neuronal network of the respiratory brainstem in mice is almost mature at the time of birth, with most synaptic contacts already established. It can therefore serve as an ideal system for the analysis of the consequences of NL deletion on synaptogenesis in the intact brain of newbom NL 1-3 triple KOs.

To examine whether the striking functional deficits in the respiratory brainstem of NL 1-3 triple KOs reflect aberrant synaptogenesis, we performed a detailed morphometric analysis of synapse types and densities at the light microscopic and ultrastructural level. Immunofluorescence staining of brainstem sections for the presynaptic marker synaptophysin 1 showed no differences in the overall density of presynaptic terminals in the $\mathrm{PBC}$, the $\mathrm{NH}$, and the neighboring inferior olive when images from control and NL 1-3 triple KO mice were compared. However, the ratio between the number of glutamatergic terminals as assessed by VGluT1/2 immunoreactivity and the number of GABAergic/glycinergic terminals as assessed by VIAAT immunoreactivity was slightly increased in the PBC and inferior olive, but not in the NH of triple KOs (Figures 6A and 6B). This change was due to a very subtle alteration in the absolute numbers of glutamatergic and GABAergic/glycinergic synapses, which was not statistically significant and became only apparent when the ratios between the numbers of the two types of synapses were analyzed.

Using immunofluorescence staining for inhibitory postsynaptic markers in the PBC, we found a $30 \%$ reduction in the number of postsynaptic clusters containing GABA $R$ a1 but no significant changes in the number of postsynaptic clusters containing gephyrin or GlyRa subunits (Figures $6 \mathrm{C}$ and $6 \mathrm{D}$ ). Moreover, the staining intensity of individual $G A B A_{A} R \propto 1$ clusters appeared to be reduced in triple KO preparations (Figure $6 \mathrm{C}$ ). Ultrastructural analysis of the PBC revealed synaptic specializations whose numbers were similar in NL 1-3 triple KO and control samples (Figures $6 \mathrm{E}$ and $6 \mathrm{~F}$ ).

In a final series of experiments, we performed double labeling experiments in order to determine the number of matched and mismatched pre- and postsynaptic glutamatergic (staining for VGluT1/NGluT2 and PSD-95) and GABAergic/glycinergic compartments (staining for VIAAT and gephyrin) in control and NL 1-3 triple KO PBC (Figure 7). We had to use an altemative fixation method for these double staining experiments, i.e., formaldehyde instead of methanol (see Experimental Procedures). As a consequence, the datasets of the single (Figures 6A-6D) and double staining experiments (Figure 7) are not directly comparable. In the case of both, glutamatergic and GABAergic/glycinergic structures, the number of "orphan" pre- or postsynaptic structures was high in the newborn PBC but very similar in control and triple KO. Likewise, the number of preand postsynaptically double labeled glutamatergic and 
A

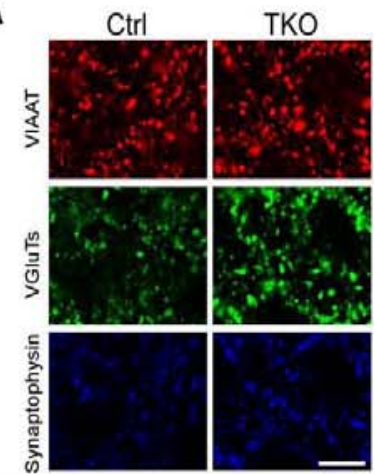

B

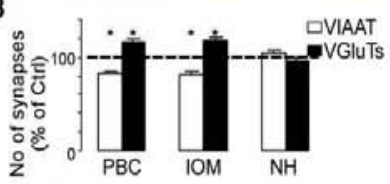

C
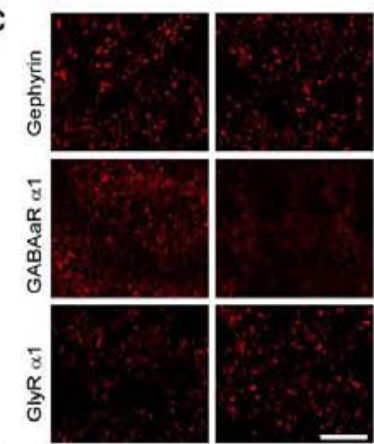

D

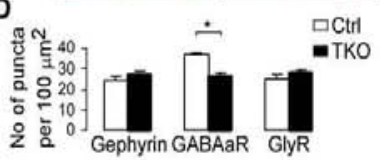

E

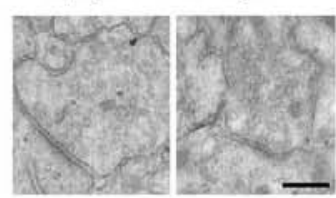

$\mathbf{F}$

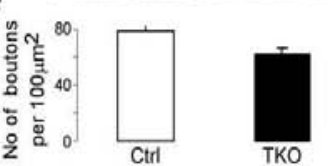

Figure 6. Altered Ratio of Glutamatergic versus GABAergic/Glycinergic Terminals but Normal Overall Synapse Numbers in the Respiratory Centers of the NL 1-3 Triple KO

(A) Representative micrographs of the PBC area of control (Ctri) and NL 1-3 triple KO (TKO) sections after triple labeling for GABAergic/ glycinergic (stained for VIAT, red). glutamatergic (stained for VGluT1/2, green), and all presynapses (stained for synaptophysin 1 , blue).

(B) Number of GABAergic/glycinergic (white) and glutamatergic (black) synapses in the PBC, inferior olive (IOM), and hypoglossal
GABAergic/glycinergic synapses was almost identical in control and triple KO PBC. Together with our morphological data on cortical explants and cultured hippocampal neurons from NL 1-3 triple KO brains (Figure 3), these findings indicate that NLs do not control the initial formation of synaptic contacts in the brain as synapse numbers remain unchanged in the absence of NLs. Consequently, the reduced expression levels of synaptic vesicle markers in NL 1-3 triple KO brains (Table 1) are unlikely to be due to prominent changes in synapse numbers, but may rather reflect aberrant protein equipment of synaptic vesicles or changes in total vesicle numbers.

\section{Discussion}

The present data show that NLs are essential for proper synapse and brain function but not for the formation of synaptic contacts per se. Loss of NLs results in a dramatic decrease in spontaneous GABAergic/glycinergic activity (Figure 4) and moderately reduced spontaneous glutamatergic activity (Figure 5) in the respiratory brainstem, which causes respiratory failure (Figure 2). The altered GABAergic/glycinergic activity in the respiratory brainstem of triple NL 1-3 KOs appears to be partly due to altered postsynaptic recruitment of $\mathrm{GABA}_{A}$ receptors (Figures $6 C$ and $6 D$ ), but a presynaptic dysfunction may also contribute (Table 1). Similarly, a combination of post- and presynaptic perturbations likely underlies the glutamatergic dysfunction in NL 1-3 triple KO brainstem. Interestingly, NLs influence the balance between glutamatergic and GABAergic/glycinergic transmission in the respiratory brainstem without affecting the total number of synapses (Figures 4-7). Triple NL 1-3 KOs exhibit an increased ratio of glutamatergic versus GABAergic/glycinergic synapses (Figures $6 A-6 D$ ) and an increase in the ratio of spontaneous glutamatergic versus GABAergic/glycinergic synaptic activity (Figure $5 D$ ) in the respiratory ihythm generating brainstem network.

\section{Synaptogenesis and Synaptic Function}

in NL 1-3 Triple KO Mice

None of our analyses on NL 1-3 triple KOs yielded data that would indicate a significant role of NLs in the initial formation of synaptic contacts in vivo. Irrespective of the preparation or brain region tested, synapse numbers were not affected by NL loss (Figures 3A, 3B, 6, and 7). Even the change in the ratio between the number of glutamatergic synapses and GABAergic/glycinergic synapses that we observed in the brainstem of NL 1-3 triple KOs (Figures $6 \mathrm{~A}$ and $6 \mathrm{~B}$ ) was caused by only very

nucleus (NH) of NL 1-3 triple KOs plotted as the percentages of glutamatergic and GABAergic/glycinergic synapses observed in the control (Ctrl) $(" p<0,001)$. The total number of synapses was not significantly different in the two experimental groups.

(C) Representative micrographs of the PBC area of control (Ctrl) and NL 1-3 triple KO (TKO) sections after labeling for gephyrin, $\mathrm{GABA}_{\alpha} R \propto 1$, and $\mathrm{Gly} R \propto 1$.

(D) Quantification of immunoreactive puncta (" $p<0.001)$.

( $E$ and F) Ultrastructural analysis of the PBC showing synaptic specializations in NL. 1-3 triple KO (TKO) and control (Ctri) preparations. (F) Quantification of synapse numbers as identified by ultrastructural analysis.

Scale bars, $10 \mu \mathrm{m}$ (A and C), $250 \mathrm{~nm}$ (E). Error bars indicate SEM. 
A

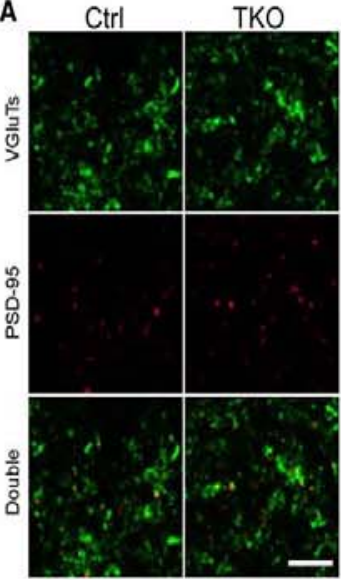

B

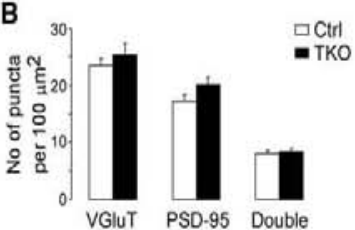

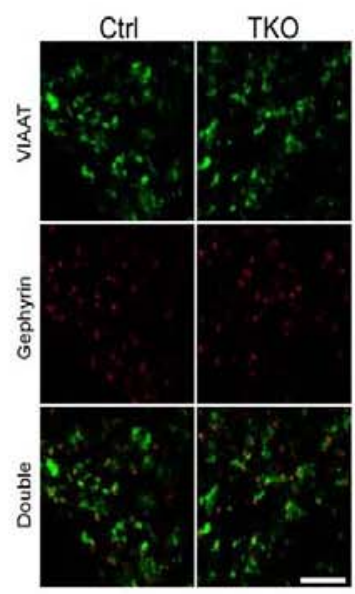

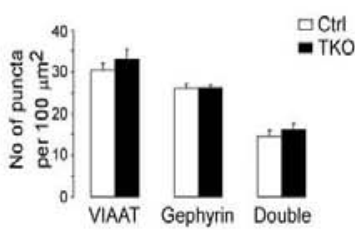

Figure 7. Properly Aligned Pre-and Postsynaptic Specializations in the Respiratory Cen ters of the NL 1-3 Triple KO

(A) The left panel shows representative micrographs of the PBC area of control (Ctrl, $n=4$ ) and NL 1-3 triple KO (TKO, $n=2$ ) sections after double labeling for glutamatergic presynapses (stained for VGluT1/2, green) and excitatory postsynapses (stained for PSD-95, red). The right panel shows representative micrographs of the PBC area of control $(C t r, n=5)$ and NL 1-3 triple KO (TKO, $n=4$ ) sections after double labeling for GABAergic/glycinergic presynapses (stained for VIAAT, green) and inhibitory postsynapses (stained for gephyrin, red).

(B) The left panel shows the quantification of isolated and colocalized VGluT1/2 and PSD-95 puncta in the PBC of control (Ctrl, white) and NL 1-3 triple KO mice (TKO, black). The right panel shows the quantification of isolated and colocalized VIAAT and gephyrin puncta in the PBC of control (Ctrl, white) and NL 1-3 triple KO mice (TKO, black).

The total number of synapses was not significantly different in the two experimenta groups. Scale bars, $30 \mu \mathrm{m}$. Error bars indicate SEM. subtle and statistically insignificant changes in absolute GABAergic/glycinergic (decrease) and glutamatergic synapse numbers (increase) in the range of $15 \%$, without affecting the total synapse number. These findings are not entirely congruent with the effects of NL knockdown in cultured neurons (Chih et al., 2005) and indicate that NLs determine functional parameters of synapses by regulating the recruitment of synaptic proteins (e.g., $\mathrm{GABA}_{\mathrm{A}}$ receptors; Figures $6 \mathrm{C}$ and $6 \mathrm{D}$ ) or organelles (e.g., synaptic vesicles; Table 1), rather than the generation of synapses per se.

At first glance, our electrophysiological studies in brainstem seem to indicate that deletion of NLs 1-3 affects inhibitory synaptic transmission more strongly than excitatory transmission. However, as mentioned above, the necessity to produce large numbers of NL 1-3 triple KOs forced us to use single NL 1 and NL 3 $\mathrm{KO}$ as well as NL $1 / 3$ double KOs as littermate controls in our electrophysiological analyses (but note that for electron microscopic [Figures $6 \mathrm{E}$ and $6 \mathrm{~F}$ ] and Westem blot studies [Table 1], wt mice with a similar mosaic genetic background were used as controls, and for light microscopic studies [Figures 3A, 3B, 6A-6D, and 7], mostly triple heterozygous littermate controls were used; see Experimental Procedures). Given that the main splice isoforms of both NL 1 (Song et al., 1999; Chih et al., 2006) and NL 3 (F.V. and S. Jamain, unpublished data) are preferentially localized to glutamatergic synapses, our approach is therefore likely to result in an underestimation of phenotypic changes in glutamatergic synapses of NL 1-3 triple KOs. A biological reason for a preferential effect of the NL 1-3 triple KO on GABAergic/glycinergic synapses may be the fact that glutamatergic synapses, in contrast to GABAergic/ glycinergic synapses, contain multiple transsynaptic cell adhesion and signaling systems that interact with intracellular scaffolding proteins (Gamer et al., 2002; Kim and Sheng, 2004; Li and Sheng, 2003; Montgomery et al., 2004; Scheiffele, 2003; Yamagata et al., 2003). These may act in parallel with the NL/NX system and may therefore be able to partially mask the effects of NL loss. This type of compensation is likely to be much less efficient in GABAergic/glycinergic synapses.

Nevertheless, our analysis of glutamatergic transmission did reveal significant functional changes in NL 1-3 triple KOs. Most notably, the frequency of glutamatergic SPSCs in the PBC of NL 1-3 triple KOs was reduced, while ePSC amplitudes were similar in all NL KO combinations (Figures $5 \mathrm{~A}-5 \mathrm{C}$ ), and the number of glutamatergic synapses was unaltered in the brainstem of NL 1-3 triple KOs (Figures $6 \mathrm{~A}, 6 \mathrm{~B}$, and 7 ). Evoked glutamatergic ePSC amplitudes in $\mathrm{NH}$ were slightly reduced in NL 1-3 triple KOs (Figures $5 \mathrm{~F}$ and $5 \mathrm{G}$ ), but cultured cortical neurons from NL 1-3 triple KOs showed no evidence of changes in glutamatergic transmisssion (Figure S3). Interestingly, our analysis of miniature glutamatergic mPSCs revealed a strongly reduced mPSC frequency but normal mPSC amplitudes in the PBC of NL 1-3 triple KOs (Figures $5 \mathrm{H}-5 \mathrm{~J}$ ). These findings, together with all other data on brainstem morphology and glutamatergic transmission, indicate that the absence of NLs $1-3$ leads to the functional shut-down of a significant number of glutamatergic synapses, hence the strong reduction in SPSC and MPSC frequency (Figures $5 \mathrm{~B}$ and $5 \mathrm{I}$ ) with no change in SPSC and $\mathrm{MPSC}$ amplitudes (Figures $5 \mathrm{C}$ and $\mathrm{5}$ ) or synapse numbers (Figures 6 and 7). This shut-down of synapses may not only be caused by postsynaptic changes but may also be due to a presynaptic dysfunction, a notion that is supported by the finding that expression levels 
of synaptic vesicle proteins are reduced in NL 1-3 triple KO brains (Table 1).

Spontaneous GABAergic/glycinergic sPSC amplitudes in the PBC and evoked GABAergic/glycinergic ePSC amplitudes in NH were both strongly reduced in NL 1-3 triple KOs (Figures $4 E$ and $4 \mathrm{H}$ ). Together with the observation that the number of postsynaptic clusters containing $G A B A_{A} R \propto 1$ were reduced while the number of postsynaptic clusters containing gephynin or GlyR $\alpha$ subunits (Figures $6 \mathrm{C}$ and $6 \mathrm{D}$ ) and the numbers of inhibitory synapses as determined by double labeling for VIAAT and gephyrin (Figure 7) were unaltered in the brainstem of NL 1-3 KOs, these data indicate that GABAergic synaptic contacts are formed initially but do not function properly in the absence of NLs, possibly due to a deficiency in the recruitment of receptors to GABAergic synapses. As seen with glutamatergic synapses, GABAergic/glycinergic mPSC frequencies are reduced but $\mathrm{MPSC}$ amplitudes are unaltered in NL 1-3 triple KO PBC (Figures $4 \mathrm{~J}-4 \mathrm{~L}$ ). This observation likely reflects a functional shut-down of a subpopulation of GABAergic synapses due to the loss of postsynaptic receptors (Figures $6 \mathrm{C}$ and $6 \mathrm{D}$ ) and/or a presynaptic dysfunction, such as a partial loss of synaptic vesicles or their aberrant equipment with proteins (Table 1).

The dramatic decrease in overall spontaneous synaptic activity in the respiratory brainstem of NL 1-3 triple KOs (Figures $4 A, 4 B, 5 A$, and $5 B$ ) is likely to be caused by the combined glutamatergic and GABAergic synaptic dysfunction. GABAergic and glycinergic transmission in the newborn respiratory brainstem is still largely excitatory (Ritter and Zhang, 2000). Consequently, a combined reduction in glutamatergic and GABAergic synaptic transmission will result in reduced excitatory drive and low overall spontaneous synaptic activity. Changes in neuronal excitability do not appear to contribute to the decreased network activity in NL 1-3 triple KOs, as neuronal $\mathrm{Na}^{+}, \mathrm{K}^{+}$, and $\mathrm{Ca}^{2+}$ currents were normal in these mice (Figure S4). Given that NLs do not regulate initial synaptogenesis per se, the increase in glutamatergic versus GABAergic/glycinergic synapse ratios observed in the PBC and inferior olive in NL 1-3 triple KOs may be partly due to homeostatic adaptations that occur as a consequence of the strongly reduced network activity.

Redundancy among NLs

At first glance, the fact that all single NL KOs as well as all combinations of $\mathrm{NL}$ double $\mathrm{KOs}$ are viable whereas NL 1-3 triple KOs die shortly after birth may indicate a significant degree of functional redundancy among NLs 1-3, which even extends to the differentially localized dominant splice isoforms of NL 1 and NL 2. However, an analysis of NL 2 distribution in NL 1 single KO neurons showed that the main NL 2 splice variant is not recruited to glutamatergic synapses when NL 1 is absent (F.V., and N.B., unpublished data), demonstrating that these NLs do not simply replace each other ectopically if one of them is eliminated. In view of these findings, we propose that the perinatal lethality of the NL 1-3 triple KOs is due to a synthetic lethal effect of the combination of the three $\mathrm{NL}$ single $\mathrm{KO}$, where subtle mutant synaptic phenotypes accumulate to cause the failure of essential brain networks, such as the respiratory system, and death.

\section{NL KO Mice versus NL Knock-Down and} Overexpression in Cultured Neurons

The present study shows that NLs are dispensable for the initial formation of synapses in cultured neurons (Figure 3) and in the intact brain (Figures 6 and 7). This finding is in agreement with data obtained in $\alpha$-NX $\mathrm{KOs}$, where loss of all three $\alpha$-NXs has very little effect on overall synapse densities (Missler et al., 2003) but, as mentioned above, does not entirely agree with results obtained in experiments involving NL overexpression or RNAi knock-down of NLs in cultured neurons (Dean et al., 2003; Graf et al., 2004; Chih et al., 2005; Levinson et al., 2005; Nam and Chen, 2005; Prange et al., 2004; Scheiffele et al., 2000). To a certain degree, the discrepancy between the present data on NL 1-3 triple KOs and the data obtained in NL overexpression experiments can be formally dismissed because the overexpression data document that NLs are sufficient to trigger synaptogenesis in vitro whereas our present $\mathrm{KO}$ study documents that they are not necessary for this process in vivo. However, even in view of these considerations, certain aspects of the discrepancy between the present data on NL 1-3 triple KOs and the data obtained after RNAi knock-down of NLs in cultured neurons remain. For example, even the knock-down of a single NL causes strong reductions in synapse numbers of cultured neurons in vitro (Chih et al., 2005) whereas KO of all NL expression has no effect on synapse numbers in cultured neurons in vitro (Figure 3 ) or in the intact brain in vivo (Figures 6 and 7 ).

We propose that one explanation for this discrepancy may be an indirect and activity-dependent homeostatic effect rather than a direct effect of NL RNAi knock-down on synaptogenesis in cultured neurons. Such activitydependent homeostatic regulation of synapse densities has been described in numerous studies (Turrigiano and Nelson, 2004) and occurs even when activity changes are very small. For example, synaptophysin 1-deficient neurons, which show no electrophysiologically measurable functional deficit (McMahon et al., 1996), generate fewer synapses than wild-type neurons in coculture (Tarsa and Goda, 2002), indicating that even subtle functional differences between neurons cause a competition situation, where functionally disadvantaged neurons maintain their synapses less efficiently. A similar situation may arise in the published cell culture experiments involving RNAi knock-down of NLs (Chih et al., 2005), where knock-down after plasmid transfection results in a mixed population of transfected and untransfected neurons that differ with respect to their NL expression and that are therefore also likely to differ functionally with regard to synaptic transmission. Neurons with different NL expression levels in this type of mixed culture compete with each other for the same target neurons. Differences in the numbers of synapses formed by these neurons may then be secondary to differences in synaptic efficacy and synapse maintenance rather than caused by a genuine synaptogenic effect of NLs. The notion that the decrease in synapse density after knock-down of NLs in cultured neurons (Chih et al., 2005) may indeed be due to an activity-dependent homeostatic effect rather than an interference with a genuine synaptogenic role of NLs is supported by the fact that knock-down of NLs leads to a reduction in 
the number of glutamatergic synapses without affecting the frequency or amplitude of spontaneous glutamatergic synaptic events. This finding indicates that knockdown of NLs in cultured neurons affects mainly labile and inactive synapses, which might even be unique to cultured neurons.

An alternative explanation for the discrepancy between the present data on NL 1-3 triple KOs and the data obtained after RNAi knock-down of NLs in cultured neurons (Chih et al., 2005) could be that off-target effects of the RNAi constructs cause perturbations of synapse stability. A recent publication showed that retraction of synapses and dendritic spines are induced by such offtarget effects of RNAi (Alvarez et al., 2006). However, Chih et al. (2005) employed state-of-the-art RNAi control experiments in their study, and their findings are therefore very unlikely to be due to off-target artifacts.

It is also possible that the differences between the present study and the published cell culture experiments on NLs, which indicated that NL levels in neurons determine synapse densities (Chih et al., 2005; Dean et al., 2003; Graf et al., 2004; Levinson et al., 2005; Nam and Chen, 2005; Prange et al., 2004; Scheiffele et al., 2000), are due to specific compensatory effects in the NL 1-3 triple KOs in vivo, e.g., through upregulation of other cell adhesion systems. However, we regard this possibility to be unlikely for the following reasons. First, the expression of NL 4, which would be the ideal compensator of a loss of NLs 1-3, is not upregulated in NL 1-3- triple KOs (Table 1). Second, dramatic homeostatic compensatory changes in postsynaptic protein expression are also known to occur in cultured cells in vitro (Ehlers, 2003). If the loss of NLs in the triple KO mice were compensated by other synaptic cell adhesion processes, thus occluding an effect of NL deletion on synaptogenesis, the same compensatory mechanisms should be relevant in cultured neurons, because the time that typically passes between overexpression or knock-down of NLs and the subsequent analysis of cultured neurons (5-6 days) is similar to the time that passes between neurogenesis and synaptogenesis in the developing brain in utero. Likewise, functional redundancy among different synaptic cell adhesion systems is a rather unlikely explanation for the finding that the KO of NLs has no effect on synaptogenesis in the brain, while RNAi knock-down of NLs reduces synapse densities in cultured cells, because the same synaptic adhesion systems are likely to operate in the two preparations.

A final possibility that needs to be considered in the context of a possible role of NLs in synaptogenesis and in view of the discrepancies between our data on NL 1-3 triple KOs and the published literature on the putative synaptogenic function of NLs in cultured neurons is that the deletion of NLs in mice does not prevent initial synaptogenesis but rather delays it. While such an effect may have been missed in our analysis of synapse densities in the brainstem, which is almost mature at birth, analyses of the immature hippocampus of newborn NL 1-3 triple KO pups also failed to detect altered synapse densities (data not shown). These data indicate that even in early phases of synapse formation the lack of NLs does not affect synapse numbers in the intact brain.
NLs and Autism

Autism is a developmental disorder that is defined behaviorally by aberrant language acquisition, perturbed social interactions, and repetitive or ritualistic behaviors. Moreover, mental retardation and epilepsy are often associated with autism. The most consistent neuroanatomical finding in autistic patients is a tendency to unusually large brains with a disproportionate contribution of white matter to the increased brain volume (Herbert, 2005). In addition, functional abnormalities such as aberrant information processing or disturbed neuronal connectivity were postulated to contribute to the autistic phenotype (Herbert, 2005; Polleux and Lauder, 2004).

Studies on affected twins and the disproportionately high number of male patients with autism led to the consensus that autism is one of the most heritable psychiatric diseases. Indeed, loss-of-function mutations in the human NL genes NLGN3 and NLGN4 cause rare monogenic heritable forms of autism (Chih et al., 2004; Comoletti et al., 2004; Jamain et al., 2003; Laumonnier et al., 2004). Consequently, the NL 3 KO mice generated in the course of the present study could become a useful genetically defined animal model of autism. The phenotypic changes in NL triple KOs described here are compatible with current hypotheses about the functional deficiencies in the brains of autistic patients, according to which "autism might be caused by an imbalance between excitation and inhibition in key neural systems" (Polleux and Lauder, 2004). However, detailed behavioral analyses will be required to assess the extent to which NL 3 KO mice model the spectrum of behavioral and cognitive phenotypes that are typically associated with autism in humans.

Experimental Procedures

Absolute NL Protein Quantification

Brains from newborn and adult mice were homogenized in $320 \mathrm{mM}$ sucrose, and protein concentrations were adjusted to a final concentration of $2 \mathrm{mg} / \mathrm{ml}$. Defined amounts of GST-NL1, -NL2, -NL3, or -NL4 fusion proteins, including the epitopes of the isoform-specific antibodies to the different NLs, were run on SDS-PAGE gels in parallel with the brain homogenates. Fusion proteins and native NLs were detected on Western blots with isoform-specific polyclonal antibodies raised against 14 amino acid peptides, follower by an ${ }^{125}$-labeled secondary antibody. After exposure to Biomax films (Kodak), bands were scanned and signal strength analyzed by densitometry (SIS).

In Situ Hybridization

Experiments were performed on paraformaldehyde-fixed $12 \mu \mathrm{m}$ thick frontal and sagittal cryostat sections prepared from freshly frozennewborn and adult rat brains, respectively (Augustin et al., 1999). Antisense oligonucleotides representing bp 917-961 and 2822-2866 of rat NL1, bp 550-594 and 2669-2713 of rat NL 2, bp 825-869, 24052449 , and $2256-2300$ of rat NL. 3 were chosen as probes and labeled with terminal transferase by using ${ }^{35} \mathrm{~S}$-dATP (accession numbers: U22952, U41662, U41663, respectively). In control experiments, hybridizations were performed with a 1000-fold excess of the respective unlabeled oligonucleotide. Sections were exposed to Kodak Biomax film for 2 weeks. Different oligonucleotides for a given $\mathrm{NL}$ isoform gave identical labeling patterns.

Protein Quantification

NL. 1-3 triple KO brains and brains from mice of a wild-type line with the same mosaic genetic background, which was derived from NL 1-3 triple KO interbreedings, were collected and immediately frozen in liquid nitrogen. Brains of the selected genotypes were 
homogeneized in $320 \mathrm{mM}$ sucrose and adjusted to a final protein concentration of $2 \mathrm{mg} / \mathrm{ml} .20 \mu \mathrm{l}$ per lane were run on SDS-PAGE gels and blotted onto nitrocellulose membranes. Several proteins (Table 1) were detected with specific primary antibodies and fluorescently labeled secondary antibodies and quantified on an Odyssey fluorescence reader (Li-Cor). Each blot was stained in parallel for a reference protein (actin or tubulin) and protein levels were expressed as the ratio over the reference protein.

Immunostaining and Light Microscopic Quantification Brains from newborn control and NL 1-3 triple KO pups were removed and either frozen by immersion in $\mathrm{N}_{2}$-cooled isopentane at $-40^{\circ} \mathrm{C}$ or immersion-fixed overnight in $4 \%$ paraformaldehyde, cryoprotected in sucrose, and frozen on dry ice. Serial frontal cryostat sections ( $12 \mu \mathrm{m}$ thick) were collected on slides. Sections from freshly frozen brains were further fixed by immersion in $4 \%$ paraformaldehyde at $4^{\circ} \mathrm{C}$ or in $-20^{\circ} \mathrm{C}$ cooled methanol for $10 \mathrm{~min}$. After blocking, paraformaldehyde-fixed sections were stained for VIAAT, VGluT1/2 (all guinea-pig, 1:3000 and 1:8000 or 1:10,000, Chemicon), and synaptophysin 1 (1:1000, SIGMA). Methanol-fixed sections were stained for GABA $A_{A} R \propto 1$ (1:20,000, J.M. Fritschy), gephyrin (1:2000, Synaptic Systems), and GlyR $\alpha 1$ (1:100, Synaptic Systems) or PSD95 (1:1000, Antibodies Inc.), VIAAT (rabbit, 1:4000, Chemicon), and VGluT1/2. All images were acquired as single layers at a zoom factor 4 using a Zeiss inverted confocal laser scanning microscope (LSM 510) with a $63 \times$ oil-immersion lens. For quantitative analyses (Figures 6 and 7 ), the gain and offset were held constant across images to allow for intensity comparisons. Images were then imported into the AnalySIS software (Soft-Imaging Systems) and puncta were quantified. For quantifications, thresholds were manually determined for each image prior to binarization, followed by a particle separation filter. The resulting image was added to the original, and a particle detection was carried out to measure particle number, area, mean intensity, and integral intensity.

Neurons were prepared from PO hippocampi and cultured as described (Dresbach et al., 2004). Neurons at DIV 21 were fixed with $4 \%$ paraformaldehyde ( 15 min on ice), thoroughly washed in PB, and further stained for synapsin (rabbit, 1:2000, Synaptic Systems) and PSD-95 (mouse, 1:1000, Antibodies Inc.). Coverslips were observed at high magnification $(100 x)$ with an upright epifluorescence Olympus BX-61 microscope. Images were acquired with a digital camera (F-View II) and analyzed with the AnalysIS software (Soft-Imaging Systerns). Labeled puncta were quantified by touch-count analysis on portions of dendrites of comparable diameter.

\section{Ultrastructural Analyses}

Brains from newbom wild-type and NL 1-3 triple KO pups were fixed by immersion in $4 \%$ paraformaldehyde/ $/ 0.1 \%$ glutaraldehyde in $0.1 \mathrm{M}$ phosphate buffer. $400 \mu \mathrm{m}$ thick vibratome sections of the brainstem were collected, washed, osmicated for $1 \mathrm{hr}(1 \%$ $\mathrm{OsO}_{4}$ in phosphate buffer), dehydrated through a graded series of ethanol and propylene oxide, and embedded in Durcupan (Durcupan ACM, Fluka) by a $48 \mathrm{hr}$ polymerization at $60^{\circ} \mathrm{C}$. Light gold ultrathin sections were cut, contrasted with uranyl acetate and lead citrate, and observed in a LEO 912AB transmission electron microscope (Zeiss). Digital images were captured with a ProScan CCD camera and analyzed with the Analysis software (Soft Imaging System).

Electrophysiological Recordings in Dissociated Cortical Neurons

Cocultures of neocortical explants and dissociated neocortical target neurons of newborn NL 1-3 triple KOs and triple heterozygous littermates were prepared as described (Mohrmann et al., 1999). After 2 weeks in vitro, recordings were performed in neurons from NL1-3 triple KO and triple heterozygous animals. AMPA receptormediated miniature postsynaptic curents (AMPA mPSCs) were recorded at $-60 \mathrm{mV}$ holding potential in the presence of $1 \mathrm{mM} \mathrm{Mg}^{2+}$, $20 \mu \mathrm{M}$ bicuculline methochloride, and $1 \mu \mathrm{M} T \mathrm{TX}$. To increase the frequency of AMPA mPSCs an elevated $\mathrm{K}^{*}$ concentration $(35 \mathrm{mM}$ ) in the extracellular solution was used. Glutamate receptor-mediated PSCs were evoked by local extracellular stimulation using widetip patch pipettes as stimulating electrodes and were recorded at $-60 \mathrm{mV}$ holding potential. Evoked NMDA and AMPA receptor- mediated PSCs were pharmacologically isolated using DNQX and D-AP5, respectively. Extracellular solutions were switched during the experiment using a whole-cell superfusion system.

Ventilation Recordings

Ventilation patterns were recorded by whole-body plethysmography, where unanesthetized newborn pups were placed in a $15 \mathrm{ml}$ closed chamber connected to a differential pressure transducer (CD15 Carrier Demodulator, ValiDyne). The analog signal of ventilation-related changes of air pressure was amplified and digitized using an AVD-converter (DigiData 3200, Axon Instruments) and analyzed using commercially available AxoTape and AxoGraph software (Axon Instruments).

Electrophysiological Recordings in Brainstem Slices All electrophysiological analyses were performed on brainstem neurons of mice whose genotype was unknown to the experimenter. Acute slices containing the pre-Bötzinger complex (PBC) and hypoglossal nucleus (NH) from newborn littermate mice were used for whole-cell recordings. The bath solution in all experiments consisted of (in mM) $118 \mathrm{NaCl}, 3 \mathrm{KCl}, 1.5 \mathrm{CaCl}_{2}, 1 \mathrm{MgCl}_{2}, 25 \mathrm{NaHCO}_{3}$, $1 \mathrm{NaH}_{2} \mathrm{PO}_{4}, 5$ glucose, pH 7.4, aerated with $95 \% \mathrm{O}_{2}$ and $5 \% \mathrm{CO}_{2}$ and kept at $28^{\circ} \mathrm{C}-30^{\circ} \mathrm{C}$. Evoked glutamatergic and GABAergic/glycinergic PSCs were recorded from hypoglossal neurons in the presence of $1 \mu \mathrm{M}$ strychnine and $1 \mu \mathrm{M}$ bicuculline or $10 \mu \mathrm{M}$ CNQX, re spectively. PSCs were evoked by $0.1 \mathrm{~Hz}$ field stimulations of axons of interneurons close to the PBC using a bipolar platinum electrode. An isolation unit IsoFlex (A.M.P.I.) with a custom-built power supply was used to apply currents of supramaximal stimulation strength (around $1 \mathrm{~mA}$ actual current near the slice as confirmed by current measurements). The pipette solution for evoked glutamatergic and GABAergic/glycinergic PSCs measurements contained (in mM) 140 Kgluconate (glutamatergic PSCs) or 140 $\mathrm{KCl}$ (GABAergic/glycinergic PSCs), $1 \mathrm{CaCl}_{2}, 10$ EGTA, $2 \mathrm{MgCl}_{2}$, $4 \mathrm{Na}_{3}$ ATP, $0.5 \mathrm{Na}_{3}$ GTP, 10 HEPES, pH 7.3. Peak amplitudes were averaged from 25 consecutive responses. To monitor changes in input resistance, current responses to a $-10 \mathrm{mV}$ voltage step $(20 \mathrm{~ms})$ from a holding potential of $-70 \mathrm{mV}$ were recorded before every fifth stimulus. In all experiments the distance between the stimulation and recording electrodes was similar between slices of different genotypes. Spontaneous GABAergic/glycinergic and glutamatergic PSCs were recorded from neurons of the $\mathrm{PBC}$ at a $\mathrm{Cl}^{-}$reversal potential of about $0 \mathrm{mV}$ in $10 \mu \mathrm{M} \mathrm{CNQX}$ or $1 \mu \mathrm{M}$ strychnine and $1 \mu \mathrm{M}$ bicuculline, respectively. Signals with amplitudes of at least two times above background noise were selected, and statistical significance was tested in each experiment. Spontaneous miniature GABAergic/glycinergic and glutamatergic PSCs were recorded as described above, but in the presence of $0.5 \mu \mathrm{M}$ tetrodotoxin (TTX). Signals with amplitudes of at least two times above background noise were selected, and statistical significance was tested in each experiment. There were no significant differences in noise levels between different genotypes. Voltage-activated currents were measured from neurons of the $\mathrm{PBC}$ with patch electrodes containing (in mM) $110 \mathrm{CsCl}_{2}, 30 \mathrm{TEA}-\mathrm{Cl}$ (for $\mathrm{Na}^{+}$and $\mathrm{Ca}^{2+}$-current), or 140 $\mathrm{KCl}$ (for $\mathrm{K}^{+}$current); $1 \mathrm{CaCl}_{2}, 10$ EGTA, $2 \mathrm{MgCl}_{2}, 4 \mathrm{Na}_{3} \mathrm{ATP}, 0.5$ $\mathrm{Na}_{3} \mathrm{GTP}, 10$ HEPES, $\mathrm{pH} 7.3$, and with $0.5 \mu \mathrm{M}$ tetrodotoxin and 200 $\mu \mathrm{M} \mathrm{Cd}^{2+}$ for blocking $\mathrm{Na}^{+}$and $\mathrm{Ca}^{2+}$ current in the bath solution. After establishing the whole-cell configuration, membrane capacitance serial and membrane resistances were estimated from current transient induced by $20 \mathrm{mV}$ hyperpolarization voltage commands from a holding potential of $-70 \mathrm{mV}$. The serial resistance was compensated by $80 \%$, and patches with a serial resistance of $>20 \mathrm{M} \Omega$, a membrane resistance of $<0.8 \mathrm{G} \Omega$, or leak currrents of $>150 \mathrm{pA}$ were excluded. The membrane currents were filtered by a fourpole Bessel filter at a corner frequency of $2 \mathrm{kHz}$, and digitized at a sampling rate of $5 \mathrm{kHz}$ using the DigiData 1200B interface (Axon Instruments). Currents were recorded and quantified as peak currents in response to voltage steps from $-70 \mathrm{mV}$ to $0 \mathrm{mV}$. The current measurements were corrected using the $\mathrm{P} / 4$ protocol that subtracts leak currents measured during four leak-subtraction prepulses applied immediately before each voltage step. Data acquisition and analysis was done using commercially available sottware (pClamp 6.0 and AxoGraph 4.6, Axon Instruments, and Prism 4 Software, GraphPad). 
Supplemental Data

The Supplemental Data for this article, including four Figures, can be found online at http://www.neuron.org/cgi/content/full/51/6/ $741 / \mathrm{DC1}$.

\section{Acknowledgments}

This work was supported by a postdoctoral fellowship and institutional support from the Max-Planck-Society (N.B.), by grants BR1107/3 and BR1107/4 from the German Research Foundation (N.B), by grant FZT103 from the German Research Foundation Center for Molecular Physiology of the Brain (N.B., W.Z.), by a postdoctoral fellowship of the German Research Foundation (M.M.), by grant R37 MH52804-08 from the National Institute of Mental Health (T.C.S.), and by the Howard Hughes Medical Institute (T.C.S.). We thank Guido Meyer (Max Planck Institute of Experimental Medicine, Göttingen) for helpful discussions, and Klaus Hellmann (Max Planck Institute of Experimental Medicine, Göttingen), Iza Komblum, Andrea Roth, Eva Borowicz (all University of Texas Southwestern Medical Center, Dallas), and the staff of the DNA Core Facility and the Transgenic Animal Facility of the Max Planck Institute of Experimental Medicine (Göttingen) for excellent technical assistance. We are grateful to Jean-Marc Fritschy (University of Zürich) for generously providing anti-GABA ${ }_{\alpha}$-receptor antibodies.

Received: January 30, 2006

Revised: July 31, 2006

Accepted: September 1, 2006

Published: September 20, 2006

References

Alvarez, V.A., Ridenour, D.A., and Sabatini, B.L. (2008). Retraction of synapses and dendritic spines induced by off-target effects of RNA interference. J. Neurosci. 26, 7820-7825.

Augustin, I., Betz, A., Herrmann, C., Jo, T., and Brose, N. (1999). Differential expression of two novel Munc13 proteins in rat brain. Biochem. J. 337, 363-371.

Biederer, T., and Südhof, T.C. (2000). Mints as adaptors. Direct binding to neurexins and recruitment of munc18. J. Biol. Chern. 275, 39803-39806.

Boucard, A.A., Chubykin, A.A., Comoletti, D., Taylor, P., and Südhof, T.C. (2005). A splice code for trans-synaptic cell adhesion mediated by binding of neuroligin 1 to alpha- and beta-neurexins. Neuron 48 , 229-236.

Chih, B., Afridi, S.K., Clark, L., and Scheiffele, P. (2004). Disorder-associated mutations lead to functional inactivation of neuroligins. Hum. Mol. Genet. 13, 1471-1477.

Chih, B., Engelman, H., and Scheiffele, P. (2005). Control of excitatory and inhibitory synapse formation by neuroligins. Science 307, 1324-1328.

Chih, B., Gollan, L., and Scheiffele, P. (2006). Alternative splicing controls selective trans-synaptic interactions of the neuroligin-neurexin complex. Neuron 51, 171-178.

Chubykin, A.A., Liu, X., Comoletti, D., Tsigelny, l., Taylor, P., and Südhof, T.C. (2005). Dissection of synapse induction by neuroligins: Effects of a neuroligin mutation associated with autism. J. Biol. Chem. 280, 22365-22374.

Comoletti, D., De Jaco, A., Jennings, L.L., Flynn, R.E., Gaietta, G., Tsigelny, I., Ellisman, M.H., and Taylor, P. (2004). The Arg451Cysneuroligin-3 mutation associated with autism reveals a defect in protein processing. J. Neurosci. 24, 4889-4893.

Dean, C., Scholl, F.G., Choih, J., DeMaria, S., Berger, J., Isacoff, E., and Scheiffele, P. (2003). Neurexin mediates the assembly of presynaptic terminals. Nat. Neurosci. 6, 708-716.

Dresbach, T., Neeb, A., Meyer, G., Gundelfinger, E.D., and Brose, N. (2004). Synaptic targeting of neuroligin is independent of neurexin and SAP90/PSD 95 binding. Mol. Cell. Neurosci. 27, 227-235.

Ehlers, M.D. (2003). Activity level controls postsynaptic composition and signaling via the ubiquitin-proteasome system. Nat. Neurosci. 6, 231-242.
El-Husseini, A., Schnell, E., Dakoji, S., Sweeney, N., Zhou, Q., Prange, O., Gauthier-Campbell, C., Aguilera-Moreno, A., Nicoll, R.A., and Bredt, D.S. (2002). Synaptic strength regulated by palmitate cycling on PSD-95. Cell 108, 849-863.

Fu, Z., Washbourne, P., Ortinski, P., and Vicini, S. (2003). Functional excitatory synapses in HEK293 cells expressing neuroligin and glutamate receptors. J. Neurophysiol. 90, 3950-3957.

Fukata, Y., Tzingounis, A.V., Trinidad, J.C., Fukata, M., Burlingame, A.L., Nicoll, R.A., and Bredt, D.S. (2005). Molecular constituents of neuronal AMPA receptors. J. Cell Biol. 169, 399-404.

Garner, C.C., Zhai, R.G., Gundelfinger, E.D., and Ziv, N.E. (2002). Molecular mechanisms of CNS synaptogenesis. Trends Neurosci. 25, 243-251.

Graf, E.R., Zhang, X., Jin, S.X., Linhoff, M.W., and Craig, A.M. (2004). Neurexins induce differentiation of GABA and glutamate postsynaptic specializations via neuroligins. Cell 119, 1013-1026.

Hata, Y., Butz, S., and Sũdhof, T.C. (1996). CASK: a novel dlg/PSD95 homolog with an $\mathrm{N}$-terminal calmodulin-dependent protein kinase domain identified by interaction with neurexins. J. Neurosci. 16, 2488-2494.

Herbert, M.R. (2005). Large brains in autism: the challenge of pervasive abnormality. Neuroscientist $11,417-440$.

Hirao, K., Hata, Y., Ide, N., Takeuchi, M., Irie, M., Yao, I., Deguchi, M., Toyoda, A., Südhof, T.C., and Takai, Y. (1998). A novel multiple PDZ domain-containing molecule interacting with $\mathrm{N}$-methyl-D-aspartate receptors and neuronal cell adhesion proteins. J. Biol. Chem. 273, 21105-21110.

Ichtchenko, K., Hata, Y., Nguyen, T., Ullirich, B., Missler, M., Moomaw, C., and Südhof, T.C. (1995). Neuroligin 1: a splice site-specific ligand for beta-neurexins. Cell 81, 435-443.

Ichtchenko, K., Nguyen, T., and Sūdhof, T.C. (1996). Structures, alternative splicing, and neurexin binding of multiple neuroligins. J. Biol. Chem. 271, 2676-2682.

Irie, M., Hata, Y., Takeuchi, M., Ichtchenko, K., Toyoda, A., Hirao, K., Takai, Y., Rosahl, T.W., and Südhof, T.C. (1997). Binding of neuroligins to PSD-95. Science 277, 1511-1515.

Jamain, S., Quach, H., Betancur, C., Rastam, M., Colineaux, C., Gillberg, I.C., Soderstrom, H., Giros, B., Leboyer, M., Gillberg, C., and Bourgeron, T. (2003). Mutations of the X-linked genes encoding neuroligins NLGN3 and NLGN4 are associated with autism. Nat. Genet. 34, 27-29.

Kim, E., and Sheng, M. (2004). PDZ domain proteins of synapses. Nat. Rev. Neurosci. 5, 771-781.

Kim, E., Niethammer, M., Rothschild, A., Jan, Y.N., and Sheng, M. (1995). Clustering of Shaker-type $K^{+}$channels by interaction with a family of membrane-associated guanylate kinases. Nature 378 , 85-88.

Kornau, H.C., Schenker, L.T., Kennedy, M.B., and Seeburg, P.H. (1995). Domain interaction between NMDA receptor subunits and the postsynaptic density protein PSD-95. Science 269, 1737-1740. Laumonnier, F., Bonnet-Brilhault, F., Gomot, M., Blanc, R., David, A., Moizard, M.P., Raynaud, M., Ronce, N., Lemonnier, E., Calvas, P., et al. (2004). X-linked mental retardation and autism are associated with a mutation in the NLGN4 gene, a member of the neuroligin family. Am. J. Hum. Genet. 74, 552-557.

Levinson, J.N., Chery, N., Huang, K., Wong, T.P., Gerrow, K., Kang, R., Prange, O., Wang, Y.T., and El-Husseini, A. (2005). Neuroligins mediate excitatory and inhibitory synapse formation: involvement of PSD-95 and neurexin-1 beta in neuroligin-induced synaptic specificity. J. Biol. Chem. 280, 17312-17319.

Li, Z., and Sheng, M. (2003). Some assembly required: the development of neuronal synapses. Nat. Rev. Mol. Cell Biol. 4, 833-841.

McMahon, H.T., Bolshakov, V.Y., Janz, R., Hammer, R.E., Siegelbaum, S.A., and Südhof, T.C. (1996). Synaptophysin, a major synaptic vesicle protein, is not essential for neurotransmitter release. Proc. Natl. Acad. Sci. USA 93, 4760-4764.

Meyer, G., Varoqueaux, F., Neeb, A., Oschlies, M., and Brose, N. (2004). The complexity of PDZ domain-mediated interactions at glutamatergic synapses: a case study on neuroligin. Neuropharmacology $47,724-733$. 
Neuron

754

Missler, M., Zhang, W., Rohlmann, A., Kattenstroth, G., Hammer, R.E., Gottmann, K., and Südhof, T.C. (2003). Alpha-neurexins couple $\mathrm{Ca}^{2+}$ channels to synaptic vesicle exocytosis. Nature 423, 939-948.

Mohrmann, R., Wemer, M., Hatt, H., and Gottmann, K. (1999). Target-specific factors regulate the formation of glutamatergic transmitter release sites in cultured neocortical neurons. J. Neurosci. 19, 10004-10013.

Montgomery, J.M., Zamorano, P.L., and Gamer, C.C. (2004). MAGUKs in synapse assembly and function: an emerging view. Cell. Mol. Life Sci. 61, 911-929.

Nam, C.I., and Chen, L. (2005). Postsynaptic assembly induced by neurexin-neuroligin interaction and neurotransmitter. Proc. Natl. Acad. Sci. USA 102, 6137-6142.

Polleux, F., and Lauder, J.M. (2004). Toward a developmental neurobiology of autism. Ment. Retard. Dev. Disabil. Res. Rev. 10, 303-317.

Prange, O., Wong, T.P., Gerrow, K., Wang, Y.T., and El-Husseini, A. (2004). A balance between excitatory and inhibitory synapses is controlled by PSD-95 and neuroligin. Proc. Natl. Acad. Sci. USA 101, 13915-13920.

Ritter, B., and Zhang, W. (2000). Early postnatal maturation of GABA $_{A}$-mediated inhibition in the brainstem respiratory rhythmgenerating network of the mouse. Eur. J. Neurosci. 12, 2975-2984.

Scheiffele, P. (2003). Cell-cell signaling during synapse formation in the CNS. Annu. Rev. Neurosci. 26, 485-508.

Scheiffele, P., Fan, J., Choih, J., Fetter, R., and Serafini, T. (2000). Neuroligin expressed in nonneuronal cells triggers presynaptic development in contacting axons. Cell 101, 657-669.

Schnell, E., Sizemore, M., Karimzadegan, S., Chen, L., Bredt, D.S., and Nicoll, R.A. (2002). Direct interactions between PSD-95 and stargazin control synaptic AMPA receptor number. Proc. Natl. Acad. Sci. USA 99, 13902-13907.

Serafini, T. (1999). Finding a partner in a crowd: neuronal diversity and synaptogenesis. Cell 98, 133-136.

Song, J.Y., Ichtchenko, K., Südhof, T.C., and Brose, N. (1999). Neuroligin 1 is a postsynaptic cell-adhesion molecule of excitatory synapses. Proc. Natl. Acad. Sci. USA 96, 1100-1105.

Sperry, R.W. (1963). Chemoaffinity in the orderly growth of nerve fiber patterns and connections. Proc. Natl. Acad. Sci. USA 50, 703-710.

Talebizadeh, Z., Lam, D.Y., Theodoro, M.F., Bittel, D.C., Lushington, G.H., and Butler, M.G. (2006). Novel splice isoforms for NLGN3 and NLGN4 with possible implications in autism. J. Med. Genet. 43, e21. Tarsa, L., and Goda, Y. (2002). Synaptophysin regulates activitydependent synapse formation in cultured hippocampal neurons. Proc. Natl. Acad. Sci. USA 99, 1012-1016.

Turrigiano, G.G., and Nelson, S.B. (2004). Homeostatic plasticity in the developing nervous system. Nat. Rev. Neurosci. 5, 97-107.

Varoqueaux, F., Jamain, S., and Brose, N. (2004). Neuroligin 2 is exclusively localized to inhibitory synapses. Eur. J. Cell Biol. 83 , 449-456.

Yamagata, M., Sanes, J.R., and Weiner, J.A. (2003). Synaptic adhesion molecules. Curr. Opin. Cell Biol. 15, 621-632.

Zhang, W., Rohlmann, A., Sargsyan, V., Aramuni, G., Hammer, R.E., Südhof, T.C., and Missler, M. (2005). Extracellular domains of alphaneurexins participate in regulating synaptic transmission by selectively affecting $\mathrm{N}$ - and $\mathrm{P} / \mathrm{Q}$-type $\mathrm{Ca}^{2+}$ channels. J. Neurosci. 25, $4330-4342$. 
Neuron, Volume 51

\section{Supplemental Data}

\section{Neuroligins Determine Synapse}

\section{Maturation and Function}

Frédérique Varoqueaux, Gayane Aramuni, Randi L. Rawson, Ralf Mohrmann,

Markus Missler, Kurt Gottmann, Weiqi Zhang, Thomas C. Südhof, and Nils Brose

Figure S1. Targeting Strategy and Detection of the NL 1, NL 2, and NL 3 Deletion Mutations

$A_{N L 1}$
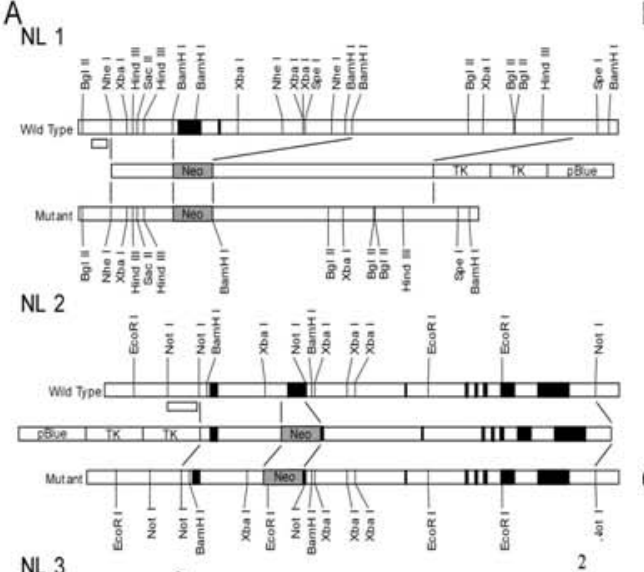

NL. 3

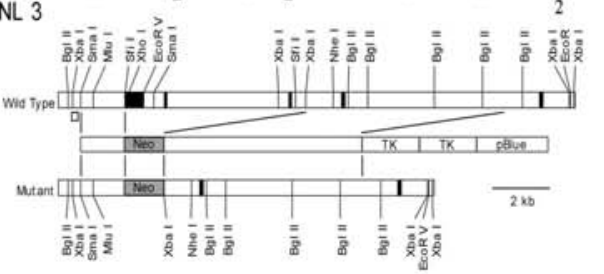

B

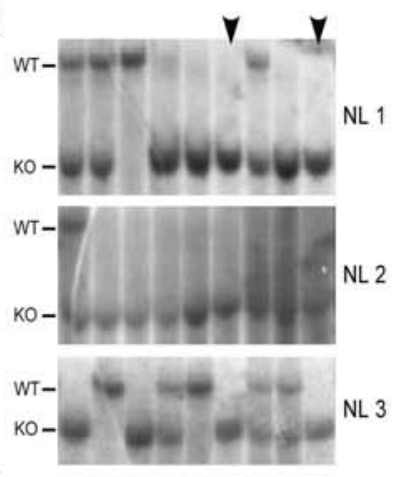

$C^{m}$

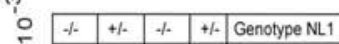

\begin{tabular}{l|l|l|l|l|l|}
\hline & + & $+/$ & $\%$ & $+/$ & Genotype NL1 \\
\cline { 2 - 6 }$\times$ & $\%$ & $+/+$ & $\%$ & $+\%$ & Genotype NL2 \\
\hline
\end{tabular}

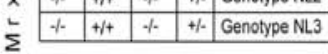

$116-$

$116-\quad-\quad$ NL 2

$116-\quad$ NL 3

(A) Representation of the targeted region of the murine NL genes, targeting vectors, and mutated genes resulting from homologous recombination. The location of the probes used for Southern analysis of genomic DNA is indicated by white bars. In all three cases, exon sequences covering the respective translational start site and $546 \mathrm{bp}, 380 \mathrm{bp}$, and $574 \mathrm{bp}$ of $5^{\prime}$ coding sequence of NL $1, \mathrm{NL} 2$, and NL 3 , respectively, were targeted for deletion by homologous recombination in embryonic stem cells.

(B) Southern blot analysis of genomic DNA from different NL genotypes. DNA was digested with $\mathrm{Bgl}$ II (for NL1 and NL3) or EcoR1 (for NL2). Bands representing the wild type (WT) and KO alleles are indicated on the left. Arrows indicate triple $\mathrm{KO}$ genotypes.

(C) Western blot analysis of adult brain homogenates from different NL genotypes. Blots were probed with isoform-specific polyclonal antibodies to the C-termini of the indicated NLs. NL 1 and NL 3 comigrate at about $116 \mathrm{kDa}$ while NL 2 is slightly smaller. 
Figure S2. Normal Brain Cytoarchitecture in the Absence of NLS

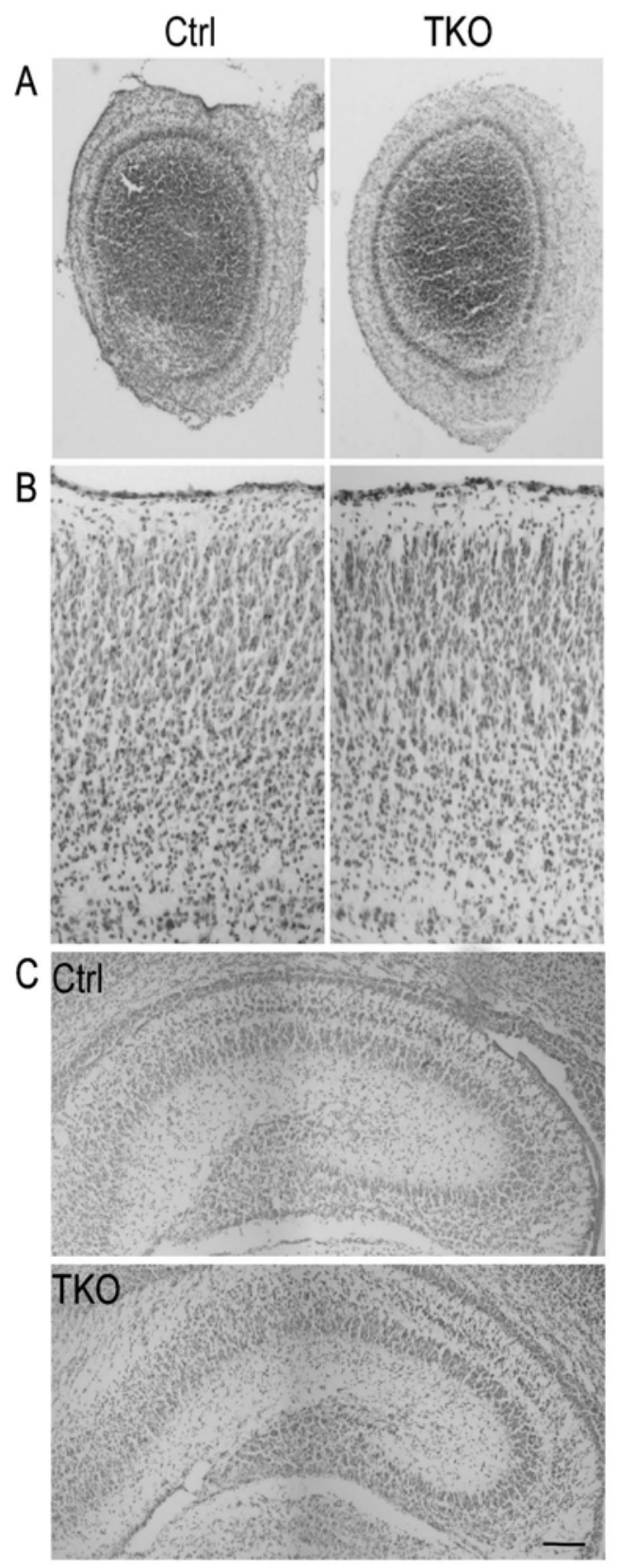

Micrographs of Nissl-stained sections taken at the level of the olfactory bulb (A), the neocortex (B) and the hippocampus (C) illustrate the normal organization of the newborn brain in the NL 1-3 triple KO (TKO) as compared to the control triple heterozygous (Ctrl) brain. Scale bar: $180 \mu \mathrm{m}(\mathrm{A})$, $20 \mu \mathrm{m}(\mathrm{B}), 60 \mu \mathrm{m}(\mathrm{C})$. 
Figure S3. Intact Glutamatergic Synaptic Transmission in Cultured Neocortical Neurons of NL 1-3 Triple KOs
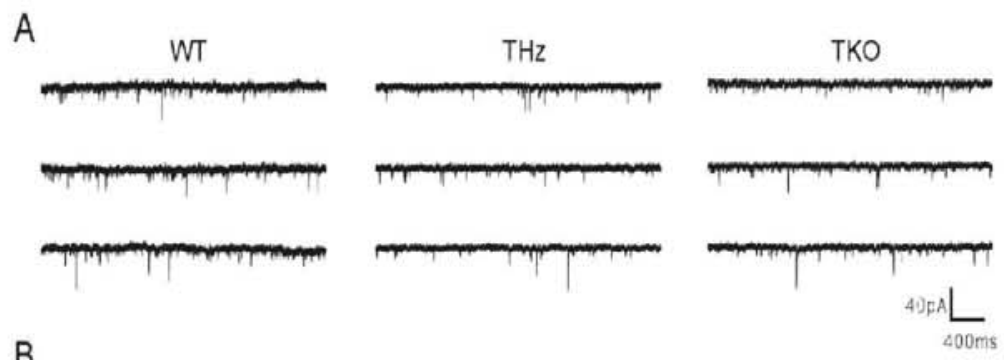

B
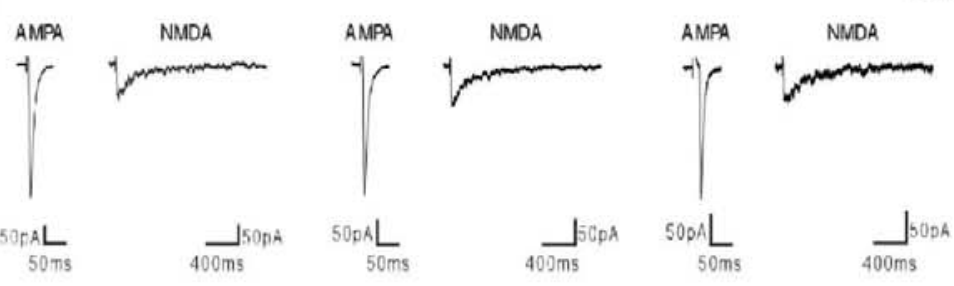

$50 \mathrm{pAL}$

$\underset{400 \mathrm{~ms}}{5 \mathrm{scps}}$

${ }_{50 \mathrm{pA}}^{50 \mathrm{~L} s}$

$\underset{400 \mathrm{~ms}}{\mathrm{Js0ph}}$
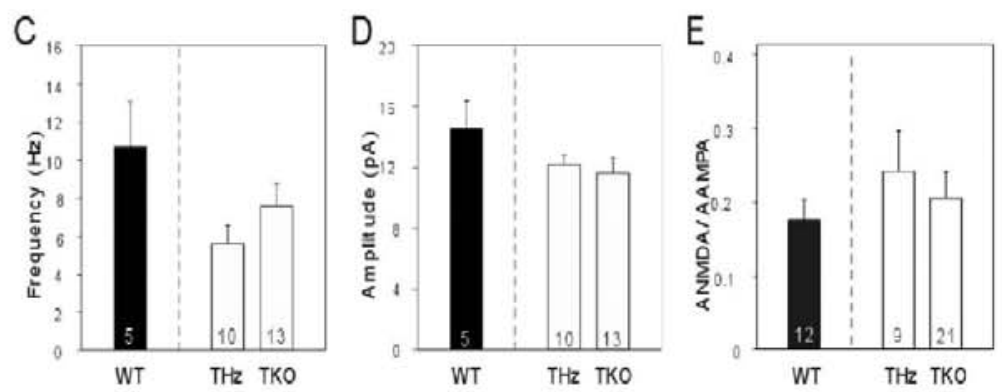

Representative example traces of AMPA receptor-mediated miniature mPSCs $(A)$ and pharmacologically isolated AMPA and NMDA receptor evoked ePSCs (B) in wild type (WT), littermate triple heterozygous control (THz) and triple $\mathrm{KO}$ (TKO) neurons. Neither the mean frequency $(C)$ nor the mean amplitude $(D)$ of AMPA mPSCs differ significantly between neurons from the different experimental groups. Furthermore, no significant differences in AMPA ePSCs, NMDA ePSCs nor in their ratio $(E)$ were observed. Numbers within the bar graphs indicate the number of neurons tested for each genotype. 
Figure S4. Unaltered Voltage-Activated Channel Function in NL 1-3 Triple Kos

A
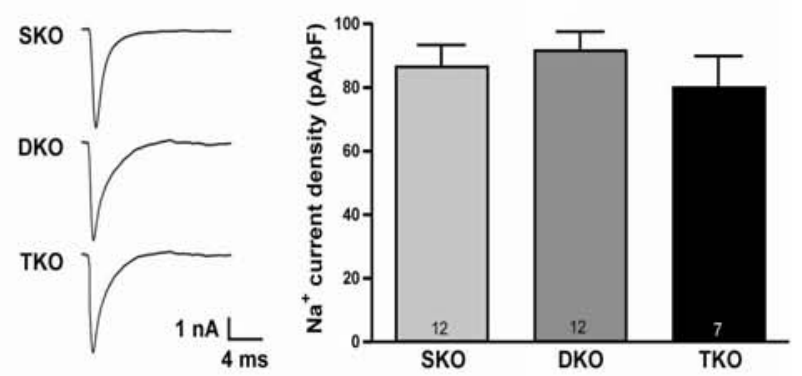

B
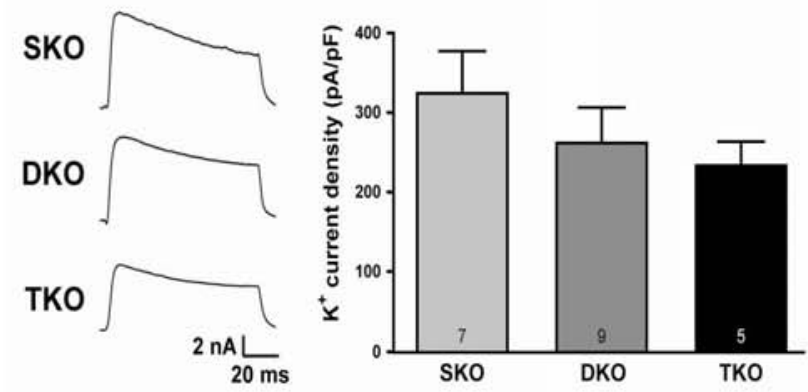

C
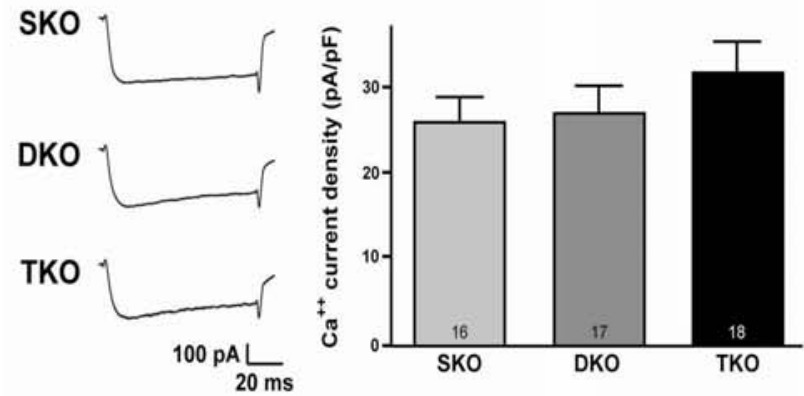

The left panels show representative whole-cell recordings of voltage-activated $\mathrm{Na}^{+}-(A) \mathrm{K}^{+}-(\mathrm{B})$ and $\mathrm{Ca}^{2+}$-currents (C) obtained from neurons in the PBC. The right panels show the corresponding bar diagrams of averaged current densities in NL 1-3 KO and control mice. Numbers within the bar graphs indicate the number of mice tested for each genotype. 


\subsection{Ventilation patterns measured by whole-body plethysmography}

In contrast to early postnatal death of NL 1-3 triple KO mice, NL 2 KO mice are viable, fertile and behaviorally normal in the cage environment. However, using whole-body pletysmography method we observed changes in the breathing pattern that did not interfere with survival of the mice. For this recordings postnatal day 1 (P1) to P3 mice were used. The respiratory rhythm was measured as frequency and coefficient of variation of ventilation. The sample traces (Fig. 3.1A) show irregular respiration pattern in neuroligin 2 mutant mice. The coefficient of variation of ventilation (CV) was significantly decreased in NL 2 $\mathrm{KO}$ mice $(0.12 \pm 0.018$ in control mice to $0.28 \pm 0.06$ in KOs; $p<0.001$; Fig $3.1 \mathrm{C})$, whereas the ventilation frequency was slightly reduced as compared to control mice $(3.2 \pm 0.26 \mathrm{~Hz}$ in control animals to $2.6 \pm 0.32 \mathrm{~Hz}$ in KOs; ns; Fig. 3.1B). These results indicate that the lack of single neuroligin gene has no significant, but influencing role on breathing of animals at P1-P3 day.

\subsection{Synaptic transmission in brainstem preBötzinger complex from neuroligin 2 mutant mice}

Considering the fact that mice lacking neuroligin 1-3 genes die after birth within few hours most likely caused by breathing disturbances, we found quite reasonable to examine the function of the brainstem respiratory network, which is essential for normal breathing. The neuronal network of respiratory system is almost mature at the time of birth, which was a perfect system for us to investigate the function of already established many synapses. We examined the synaptic function of brainstem respiratory network performing experiments in acute brainstem slices of $\mathrm{NL} 1-3$ triple $\mathrm{KO}$ newborn mice consisting of preBötzinger complex (PBC) and hypoglossal nucleus $(\mathrm{NH})$, using whole-cell

patch clamp recordings. The whole data set from these recordings showed the strong impairment of synaptic activity in the respiratory brainstem of NL 1-3 KO mice (Varoqueaux, Aramuni, et al., 2006). 
A

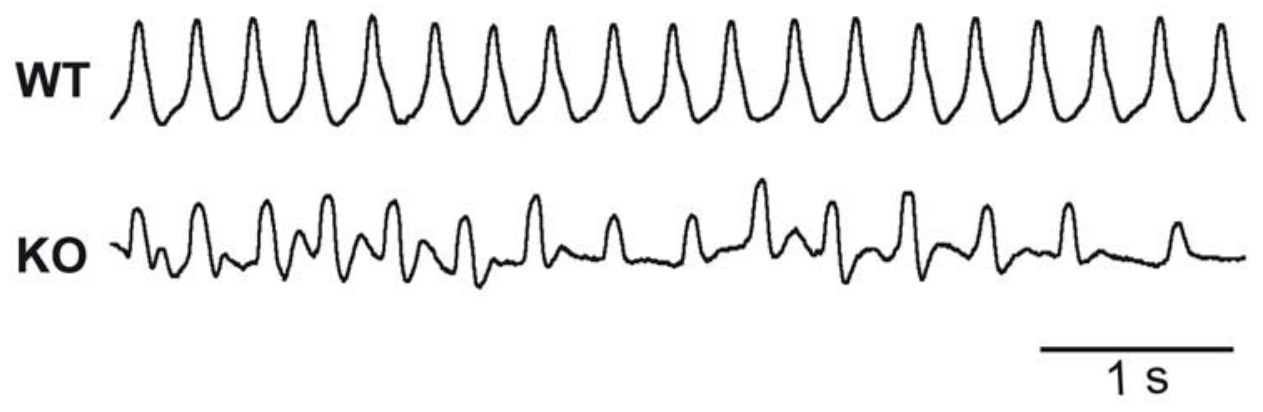

B

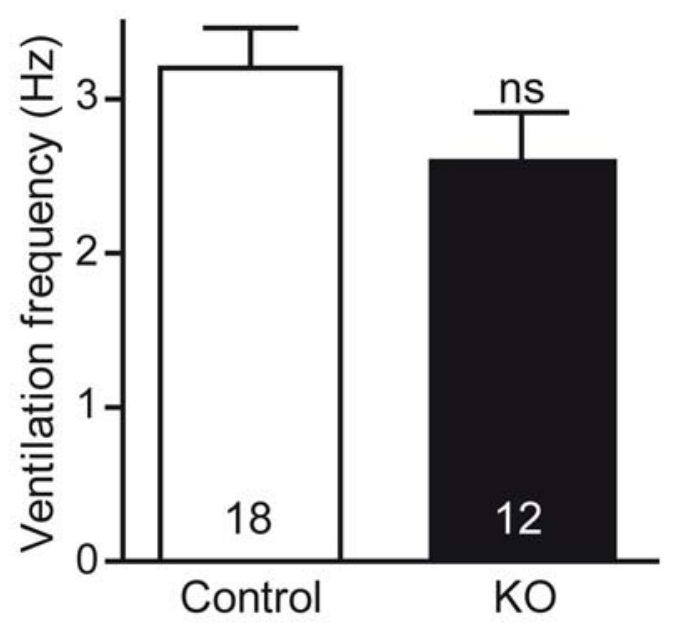

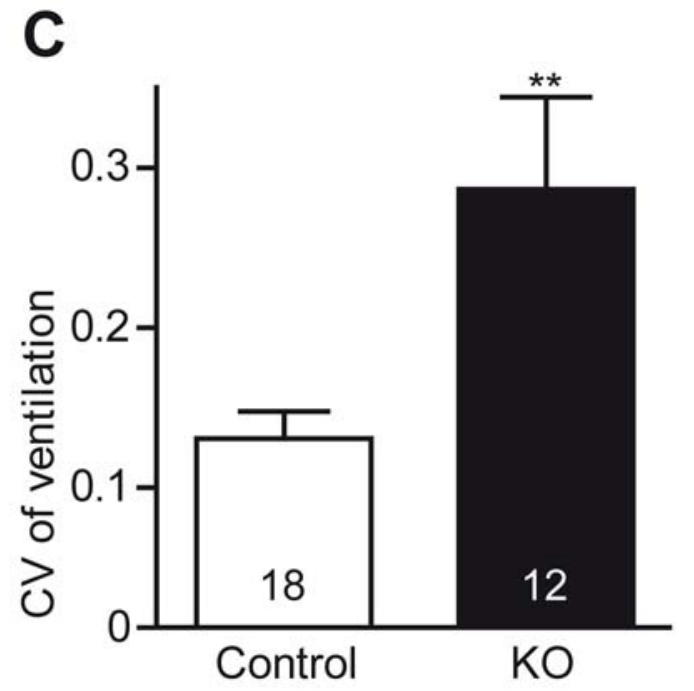

Figure 3.1Irregular ventilation patterns in NL 2 KO mice.

Representative ventilation traces $(\mathrm{A})$ and averaged ventilation frequencies $(\mathrm{B})$ and coefficient of variation (CV) of ventilation (C) of WT and NL 2 mutant mice as measured by whole-body plethysmography. Numbers within graph bars indicate the number of mice tested for each genotype. Error bars indicate SEM. NS, nonsignificant.

These results turned us to observe the role of single neuroligin gene in synaptic function. For this purpose the mice lacking neuroligin 2 were used for further experiments. Viability of these mice gave us a possibility to perform experiments at different postnatal ages. For different experiments postnatal day 0 (P0) to P5 pups were used. Before experiments the genotypes were unknown to experimenter. 


\subsubsection{Decreased spontaneous postsynaptic current in brainstem PBC neurons of NL 2 mutant mice}

NL 2 being a one of the heterotypic cell-adhesion molecules of neuroligin family has its unique important role in synaptic activity, despite the structural similarities to other members of this family (Ichtchenko et al., 1996, Irie et al., 1997). To test weather synaptic function is impaired in the brainstem of NL $2 \mathrm{KO}$ mice, we first thought to examine the total spontaneous synaptic transmission using whole-cell patch clamp recordings. Recording the spontaneous postsynaptic currents from $\mathrm{PBC}$ neurons of control and NL $2 \mathrm{KO}$ mice, we found siginificant reduction in both sPSC frequency $(5.22 \pm 0.077 \mathrm{~Hz}$ in control to $1.7 \pm 0.082 \mathrm{~Hz}$ in NL $2 \mathrm{KO}$ mice; $p<0.0001$; Fig. 3.2B) and amplitude (91.8 $\pm 0.97 p A$ in control cells to $55.8 \pm 0.83$ pA in KOs; $p<0.0001$; Fig. 3.2C) in NL 2 mutant mice compared to controls.

\subsubsection{Impaired inhibitory synaptic transmission in NL 2 KO mice}

\subsubsection{Spontaneous inhibitory postsynaptic current}

We next analyzed inhibitory (GABAergic/glycinergic) synaptic transmission in brainstem acute slices, containing PBC neurons. As we had initially predicted according to many previous studies (Varoqueaux et al., 2004, Chih et al., 2005, Prange et al., 2004) that neuroligin 2 is localized to inhibitory synapses in mammalian brain, inhibitory synaptic function in mice lacking NL 2 was strongly impaired. Recordings of spontaneous GABAergic/glycinergic postsynaptic currents were performed in the presence of $10 \mu \mathrm{M} C N Q X$ and $10 \mu \mathrm{M} \mathrm{AP5}$, in order to block glutamatergic synaptic transmission. The deletion of NL 2 gene dramatically decreased the frequency of inhibitory spontaneous PSCs by more than $60 \%(6.2 \pm 0.07 \mathrm{~Hz}$ in control to $1.95 \pm 0.07 \mathrm{~Hz}$ in NL $2 \mathrm{KO}$ in mice; $\mathrm{P}<0.0001$; Fig. 3.3B), and the amplitude was diminished about $30 \%(85.06 \pm$ $0.58 \mathrm{pA}$ in control to $59.6 \pm 1.18 \mathrm{pA} ; \mathrm{P}<0.0001$; Fig. $3.3 \mathrm{C}$ ). 


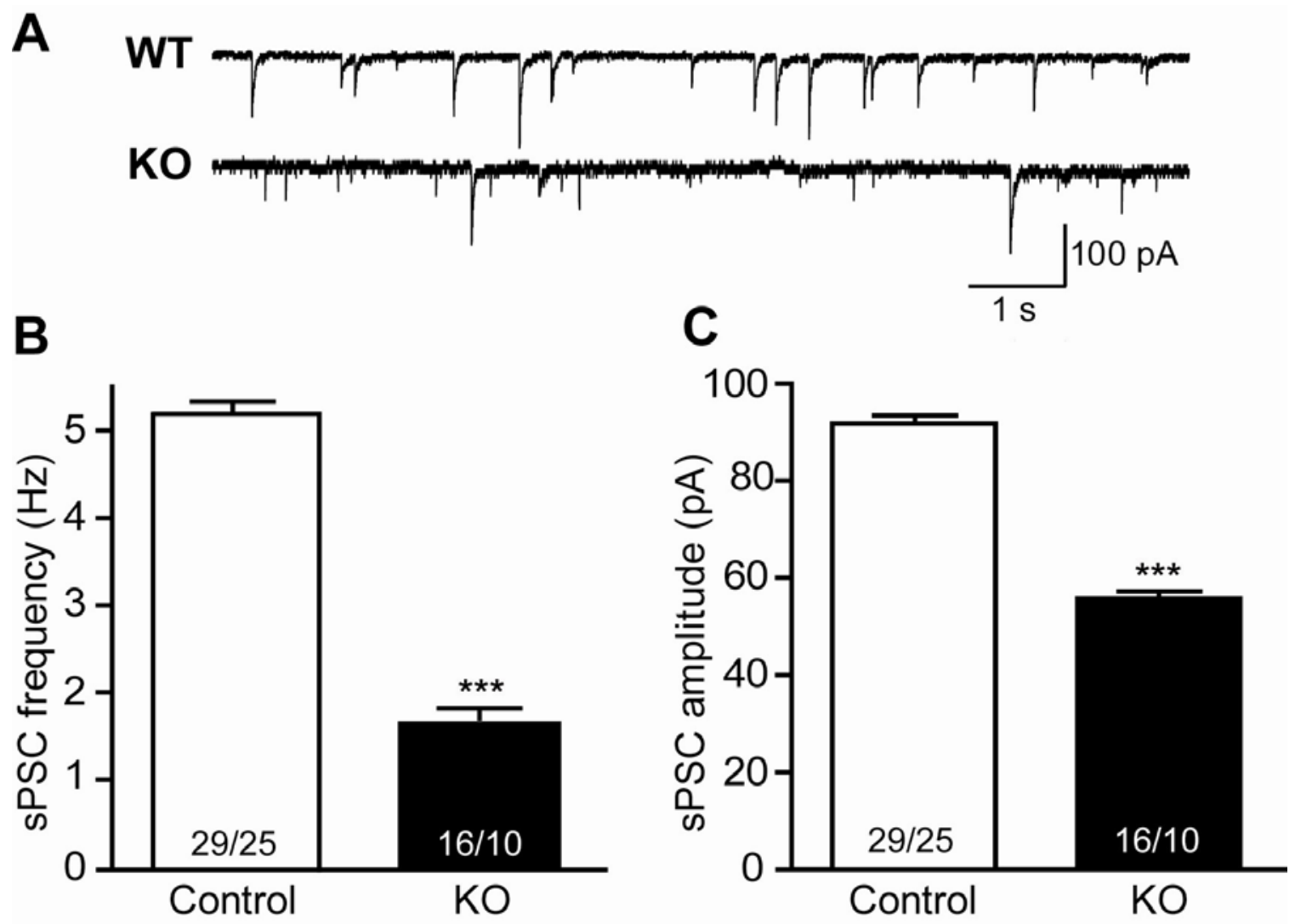

Figure 3.2 Diminished synaptic transmission in NL 2 KO mice.

(A) Sample recordings of spontaneous postsynaptic currents from littermate control and NL 2 KO mice. Impaired frequency (B) and amplitude (C) of total spontaneous PSCs in brainstem preBötzinger complex neurons. Numbers within bar graphs indicate number of cells and mice tested for each genotype. Error bars indicate SEM.

\subsubsection{Miniature spontaneous inhibitory postsynaptic currents}

The following step was to analyze the properties of pharamacologocally isolated spontaneous miniature GABAergic/glycinergic postsynaptic currents (minis) in the preBötzinger complex in acute brainstem slices from NL 2 mutant mice using whole-cell recordings in the presence of $10 \mu \mathrm{M}$ CNQX ,10 $\mu \mathrm{M}$ AP5 and $0.5 \mu \mathrm{M}$ tetrodoxin (a $\mathrm{Na}^{+}$channel blocker, which blocks action potential dependent network activity). Similar to frequency and amplitude of inhibitory SPSCs, the deletion of neuroligin 2 gene caused a dramatic decrease in frequency (1.38 \pm $0.11 \mathrm{~Hz}$ in control to $0.29 \pm 0.079 \mathrm{~Hz}$ in $\mathrm{KO}$ cells; $\mathrm{p}<0.0001$; Fig. 3.4B) and moderate reduction in amplitude $(61.58 \pm 2.08 \mathrm{pA}$ in control to $42.75 \pm 2.41 \mathrm{pA}$ in $\mathrm{KO}$ mice; $p<0.0001$; Fig. $3.4 \mathrm{C}$ ) of spontaneous miniature GABA/glcinergic 
A

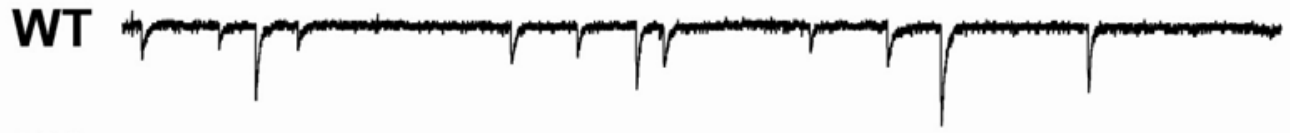

KO

B

C
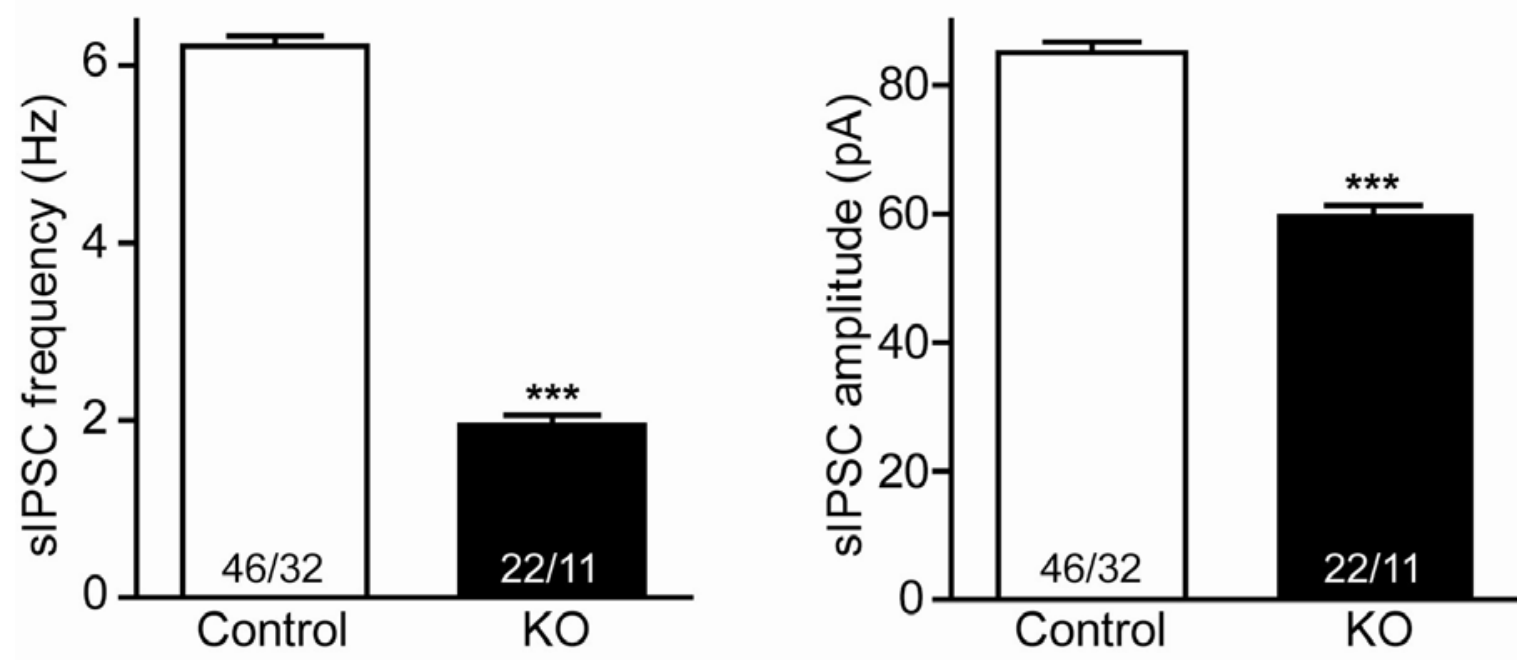

Figure 3.3 Impaired spontaneous GABA/glycinergic postsynaptic currents in NL 2 mutant mice. Representative recordings (A) of pharmacologically isolated spontaneous inhibitory postsynaptic currents in PBC neurons. Frequency (B) and amplitude (C) of sIPSCs in brainstem preBötzinger neurons are significantly decreased in NL 2 mutant mice compared to control mice. Numbers within bar graphs indicate number of cells and mice tested for each genotype. Error bars indicate SEM.

postsynaptic currents. In these experiments the strong decrease of frequency and amplitude of spontaneous minis in NL 2 KOs as compared to control cells could indicate general defect in the presynaptic terminal function and altered properties of postsynaptic receptors. 
A

WT

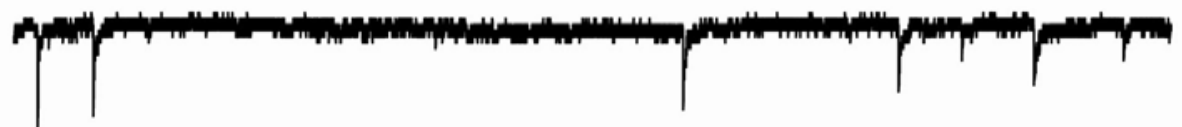

KO

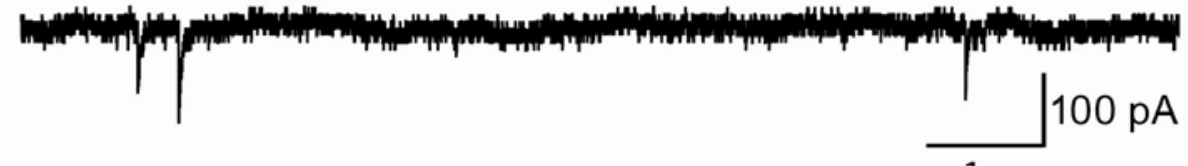

B

C

$1 \mathrm{~s}$
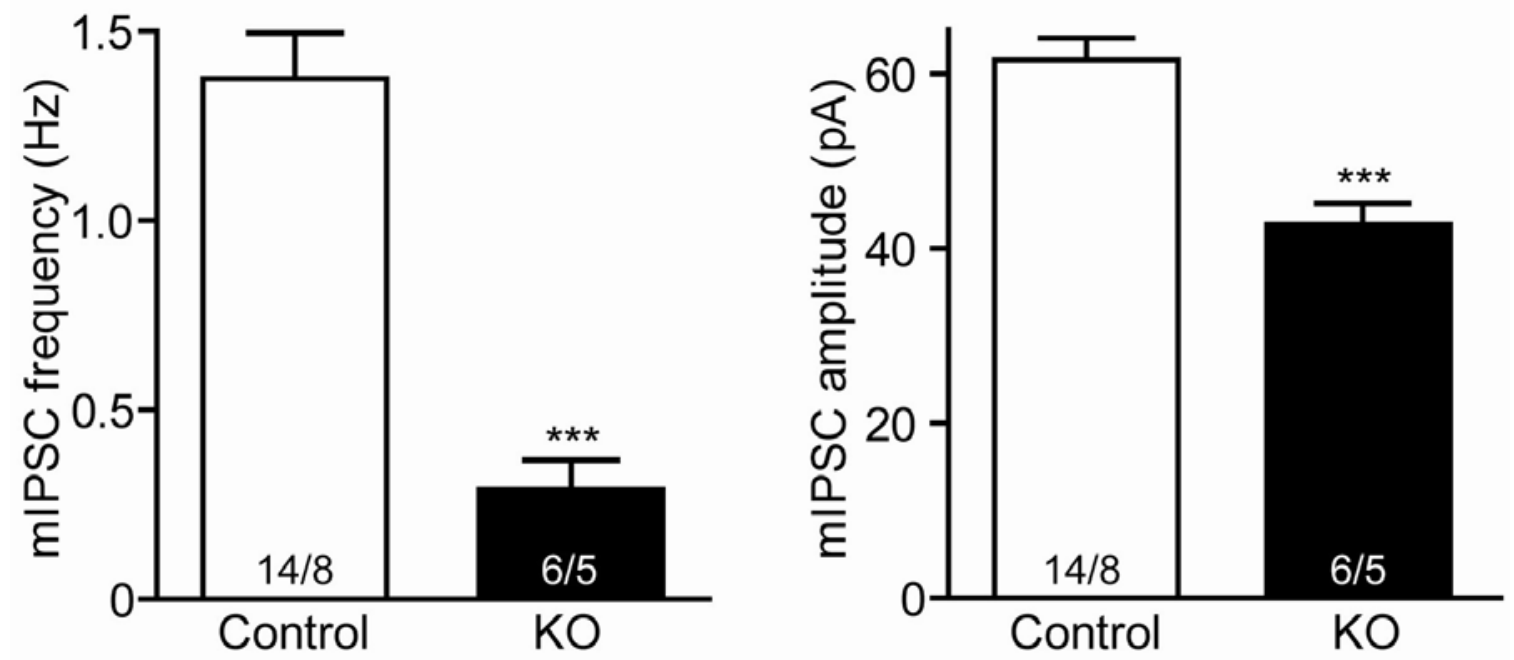

Figure 3.4 Dramatically decreased spontaneous miniature GABA/glycinerrgic PSCs.

Sample recordings $(A)$, frequency $(B)$ and amplitude $(C)$ of pharmacologically isolated miniature postsynaptic currents in PBC neurons. The frequency and amplitude of GABAergic/glycinergic mPSCs are impaired in NL 2 KO mice compared to controls. Numbers within bar graphs indicate number of cells and mice tested for each genotype. Error bars indicate SEM.

\subsubsection{Hypertonic sucrose application}

For eliciting hypertonic responses (Rosenmund and Stevens, 1996, Schlüter et al., 2004) sucrose was ejected to the neuron with 500 mbar for $500 \mathrm{~ms}$. We recorded miniature inhibitory synaptic transmission induced by $300 \mathrm{mM}$ sucrose in PBC neurons of littermate control and NL $2 \mathrm{KO}$ mice in presence of $10 \mu \mathrm{M}$ CNQX, $10 \mu \mathrm{M}$ AP5 and $0.5 \mu \mathrm{M}$ TTX. Hypertonic sucrose directly stimulates the secretory apparatus independent of $\mathrm{Ca}^{2+}$ (Rosenmund and Stevens, 1996). Deletion of neuroligin 2 moderately decreased the frequencie of miniature inhibitory postsynaptic currents induced by $300 \mathrm{mM}$ 
A

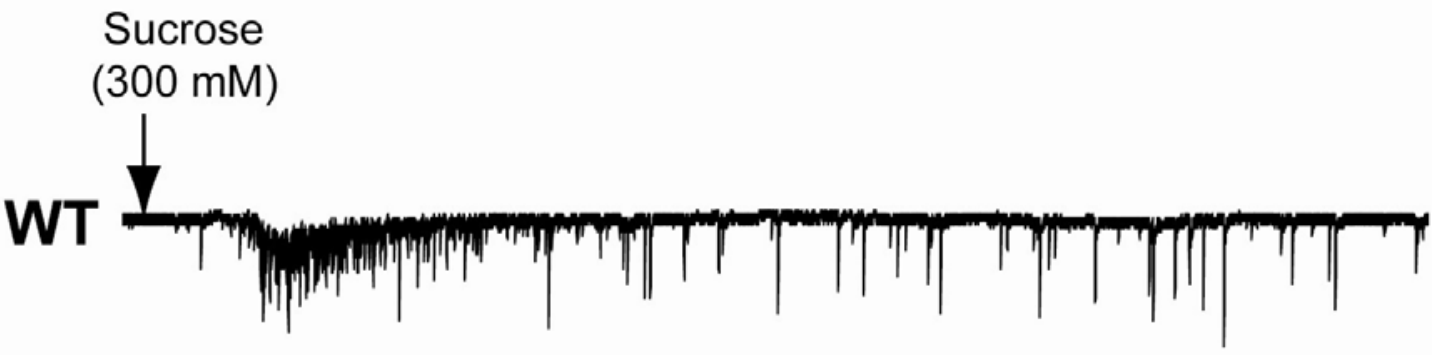

KO

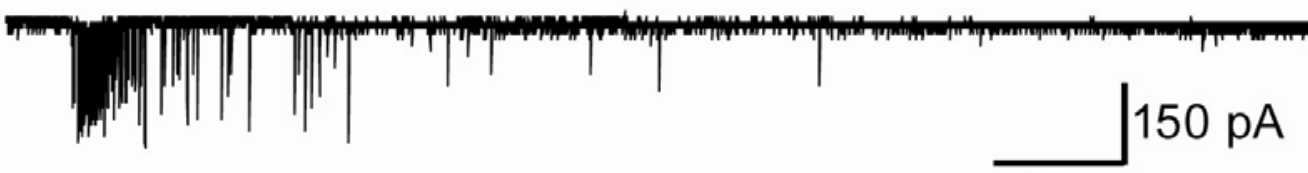

$10 \mathrm{~s}$

B

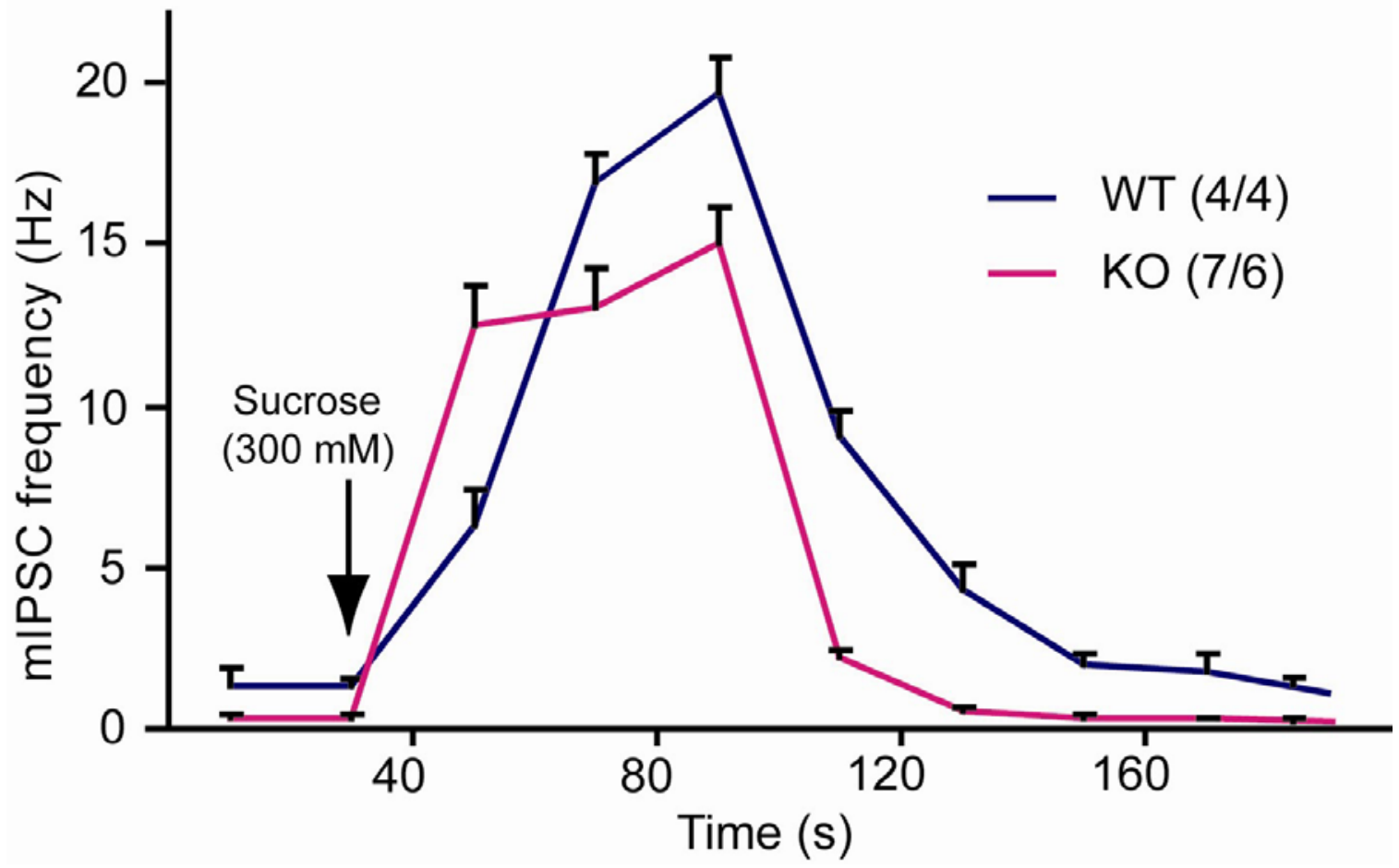

Figure 3.5 Release of GABAergic/glycinergic mPSCs by application of hypertonic sucrose.

(A) Representative traces of minitaure GABAergic/glycinergic postsynaptic currents after direct application of $300 \mathrm{mM}$ hypertonic sucrose with 500 mbar for $500 \mathrm{~s}$. After sucrose application the total frequency of mIPSCs and duration of synaptic responses (B) is diminished in NL 2 mutant compared to control mice. Numbers within bar graphs indicate number of cells and mice tested for each genotype. Error bars indicate SEM.

sucrose (Fig. 3.5A, B). Comparing the frequency of mIPSCs induced by hypertonic sucrose between control and NL 2 mutant cells, we saw that the averaged frequency after sucrose application was decreased $14 \%$ in KO mice 
(13.06 $\pm 0.48 \mathrm{~Hz}$ in control to $11.31 \pm 0.54 \mathrm{~Hz}$ in NL $2 \mathrm{KO}$ mice; $p<0.01)$. Analyzing the frequency of sucrose mediated mIPSCs during three minutes of recording we found the following: the duration of whole-cell synaptic responses elicited by hypertonic sucrose was shorter in $\mathrm{NL} 2 \mathrm{KO}$ mice, except this the response to sucrose started earlier in NL $2 \mathrm{KO}$ cells compared to controls.

\subsubsection{Deletion of NL 2 causes severe reduction of GABAergic synaptic transmission}

\subsubsection{Decreased spontaneous GABAergic PSCs in NL 2 KO mice}

The important role of GABAergic and glycinergic inhibitions in stabilizing the rhythmic network activity is indisputable (Richter and Spyer, 2001), malfunction of which shows caused the early postnatal death of NL 1-3 triple KO mice. In accordance with this we examined the role of NL 2 in inhibitory synaptic transmission and observed its importance in inhibitory function, but arose then the question whether GABAergic or glycinergic inhibition is affected in mice lacking neuroligin 2. To solve this question we performed recordings of GABAergic sPSCs in PBS neurons in brainstem acute slices having $10 \mu \mathrm{M}$ $\mathrm{CNQX}, 10 \mu \mathrm{M}$ AP 5 and $1 \mu \mathrm{M}$ strychnine in extracellular solution. Deletion of NL 2 dramatically decreased the frequency of GABAergic SPSC by more than $80 \%$ from $6.12 \pm 0.11 \mathrm{~Hz}$ in control to $0.94 \pm 0.16 \mathrm{~Hz}$ in NL 2 deficient mice $(p<0.0001$; Fig. 3.6B), while the amplitude was reduced $28 \%$ in NL 2 KOs mice (54.22 \pm $0.54 \mathrm{pA}$ in control cells to $39.27 \pm 1.7 \mathrm{pA}$ in $\mathrm{KO}$ cells; $p<0.0001$; Fig. $3.6 \mathrm{C}$ ).

\subsubsection{Impaired spontaneous miniature GABAergic PSCs in NL 2 KO mice}

To examine GABAergic minis we performed experiments in PBC neurons in presence of additional $0.5 \mu \mathrm{M}$ tetrodoxin (TTX) in extracellular bath solution. Similar to frequency and amplitude of GABAergic sPSCs (Fig. 3.6B, C), the frequency of GABAergic miniature postsynaptic currents was reduced about 
A

\section{WT}

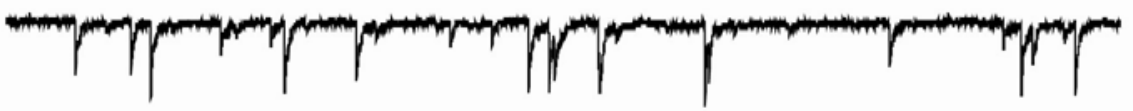

KO

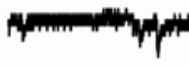

B

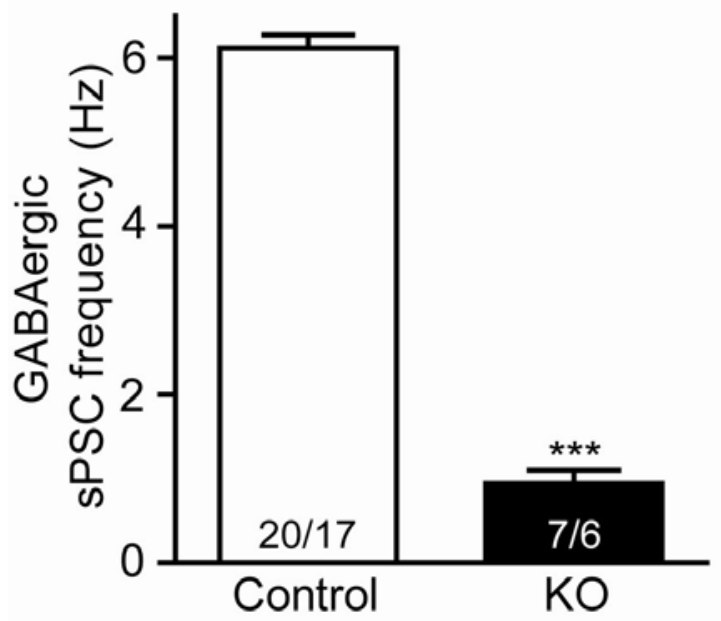

C

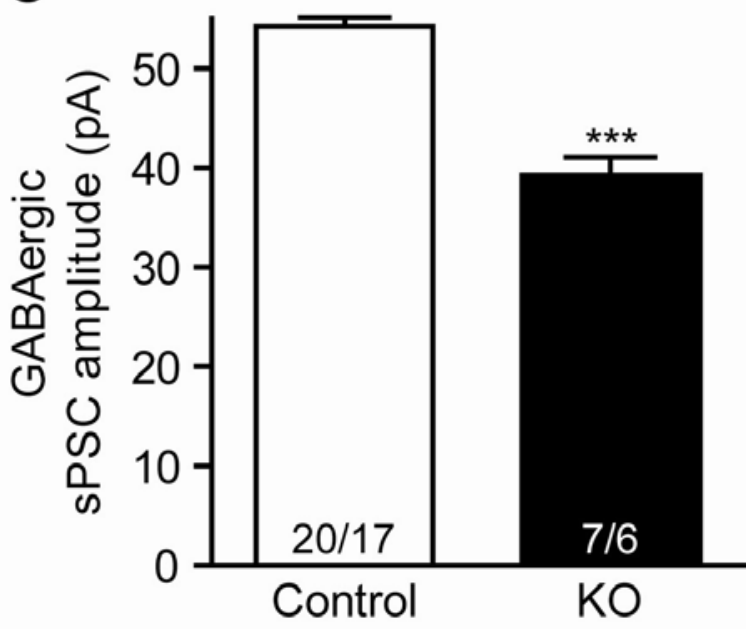

Figure 3.6 Impaired spontaneous GABAergic neurotransmission in the respiratory brainstem of the NL 2 KO mice.

(A) Representative traces of pharmacologically isolated spontaneous GABAergic postsynaptic currents in brainstem PBC neurons. Slices were prepared from littermate control and NL 2 KO mice. The lack of neuroligin 2 leads to strong reduction of frequency $(B)$ and amplitude $(C)$ of GABAergic sPSCs in PBC neurons. Numbers within bar graphs indicate number of cells and mice tested for each genotype. Error bars indicate SEM.

$80 \%(1.07 \pm 0.13 \mathrm{~Hz}$ in control cells to $0.23 \pm 0.03 \mathrm{~Hz}$ in KOs cells; $p<0.0001$;

Fig. 3.7B) and the amplitude was diminished $29 \%$ in neuroligin deficient mice as compared to controls $(60.53 \pm 2.08 \mathrm{pA}$ in control to $43.06 \pm 1.7 \mathrm{pA}$ in NL 2 mutant mice; $p<0.0001$; Fig. 3.7C). 
A
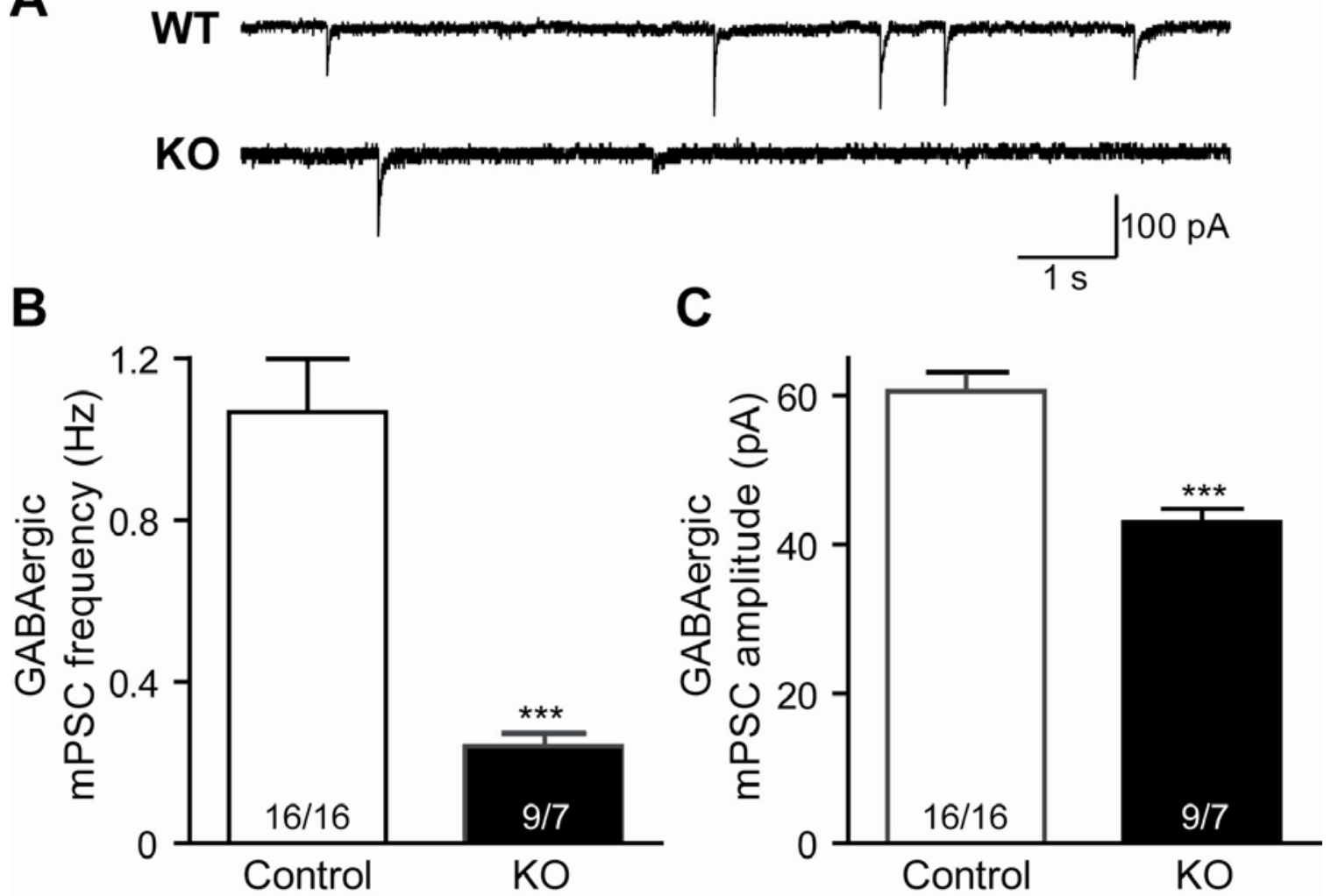

Fig.3.7 GABAergic postsynaptic currents in presence of tetrodoxin (TTX).

(A) Representative recordings of GABAergic mPSCs in brainstem neurons. Strongly reduced frequency (B) and moderately decreased amplitude (C) of GABAergic mPSCs in neuroligin $2 \mathrm{KOs}$ compared to control neurons. Numbers within bar graphs indicate number of cells and mice tested for each genotype. Error bars indicate SEM

\subsubsection{Application of $\mathrm{GABA}_{\mathrm{A}}$-receptor agonist muscimol by pressure ejection}

The reduction in amplitude of spontaneous and miniature GABAergic postsynaptic currents caused by NL 2 deletion was one of the reasons to perform further experiments with direct application of $\mathrm{GABA}_{\mathrm{A}^{-}}$receptor agonist muscimol. To block glutamatergic and glycinergic postsynaptic currents these experiments were carried out in presence of $10 \mu \mathrm{M} \mathrm{CNQX}, 10 \mu \mathrm{M}$ AP5 and $1 \mu \mathrm{M}$ strychnine (in extracellular solution), respectively. TTX $(0.5 \mu \mathrm{M})$ was added into bath solution to block action potential network activity. Direct pressure application of the $5 \mathrm{mM}$ muscimol with 500 mbar for $500 \mathrm{~ms}$ to the brainstem neuron elicited different responses in control and NL 2 mutant mice (Fig.3.8A, B). The response evoked 
A

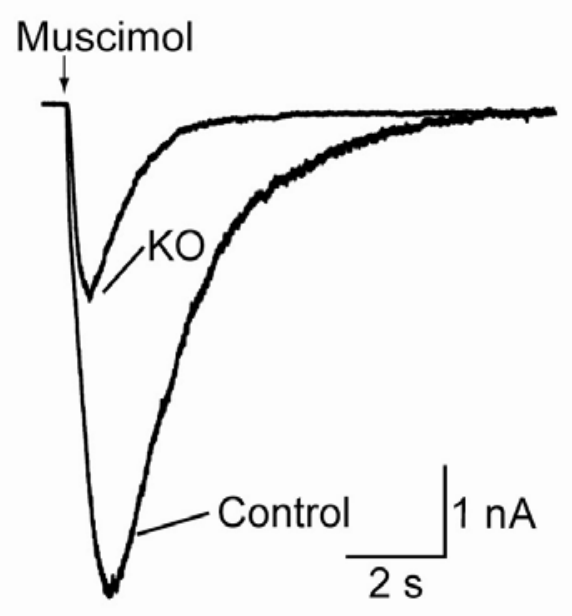

B

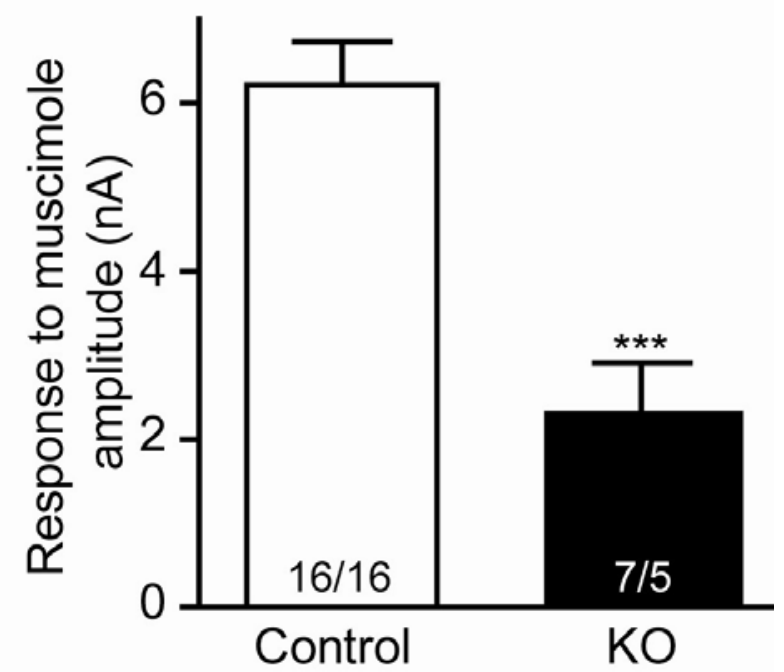

Figure 3.8 The synaptic response to local application of muscimol.

(A) Sample traces after muscimol application (with 500 mbar, $500 \mathrm{~ms}$ ) in control and NL 2 deficient mice. (B) Response to muscimol is strongly decreased in NL 2 KOs compared to control mice. Numbers within bar graphs indicate number of cells and mice tested for each genotype. Error bars indicate SEM

after muscimol application was decreased in NL 2 KO mice by more than $60 \%$ $(6.22 \pm 0.51 \mathrm{nA}$ in controls to $2.30 \pm 0.6 \mathrm{nA}$ in KOs; $\mathrm{p}<0.0001$; Fig $3.8 \mathrm{~B})$ as compared to controls.

\subsubsection{Glycinergic synaptic transmission is affected in NL 2 KO mice}

\subsubsection{Deletion of NL 2 affects spontaneous glycinergic postsynaptic currents}

As mentioned above the glycine also plays an important role in rhythmic network activity. Regarding to this and as well as to our results of ventilation recordings by whole-body plethysmography we next examined the glycinergic synaptic transmission in the same brain area. The recordings of spontaneous glycinergic

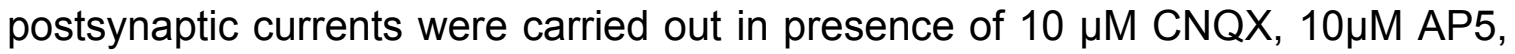
and in order to inhibit GABAergic postsynaptic currents we added $1 \mu \mathrm{M}$ bicuculline into the bath solution. The frequency and amplitude of spontaneous glycinergic postsynaptic currents were decreased markedly. In NL 2 KO mice the 


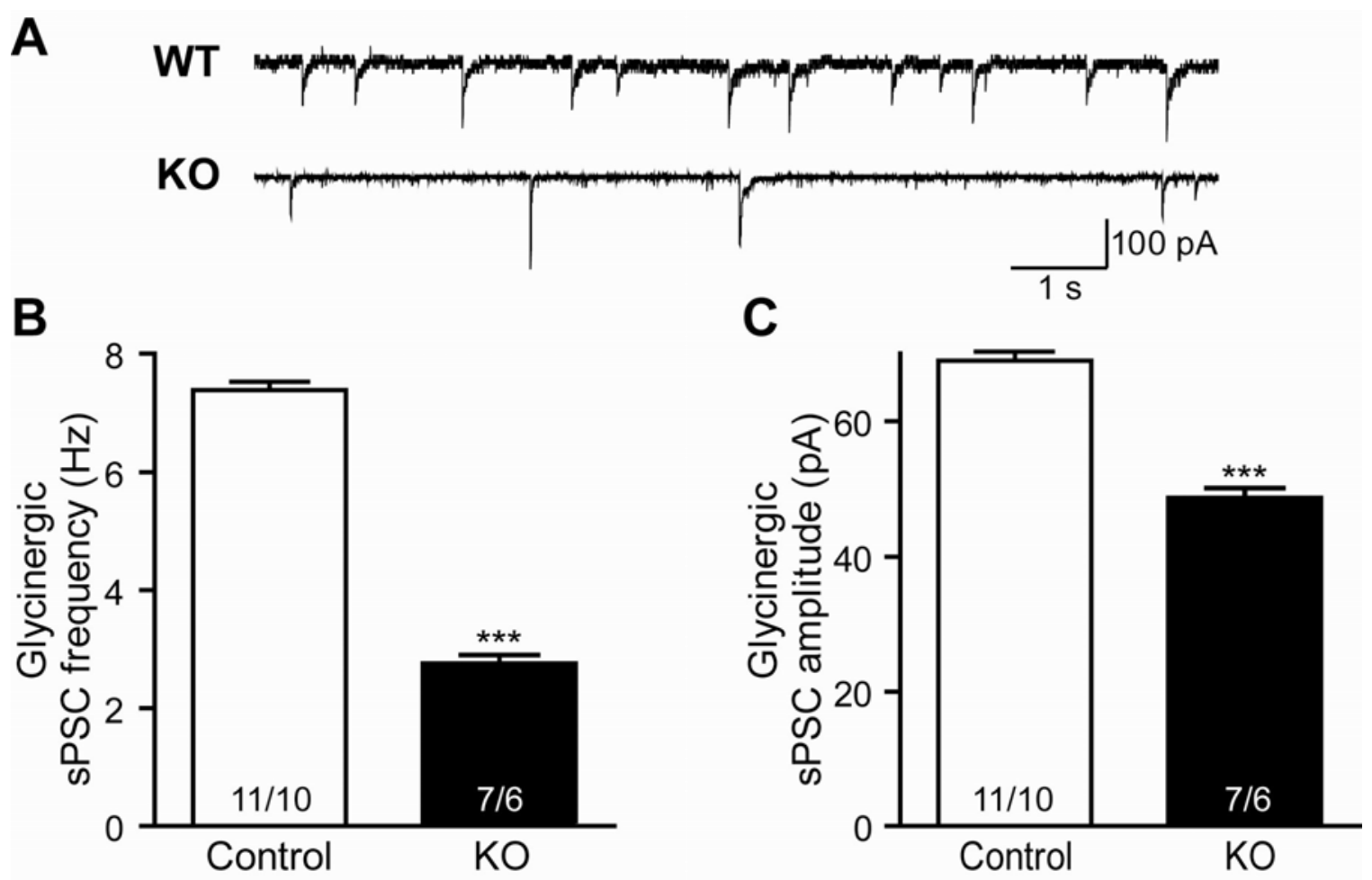

Figure 3.9 Glycinergic sPSC is reduced in NL 2 mutant mice.

(A) Representative recordings of glycinergic SPSCs. Both the frequency (B) and amplitude (C) of glycinergic sPSCs are decreased in NL 2 mutant mice. Numbers within bar graphs indicate number of cells and mice tested for each genotype. Error bars indicate SEM.

frequency of glycinergic sPSCs compared to frequency of spontaneous GABAergic postsynaptic currents (Fig. 3.6 A, B) was affected less, about $60 \%$ (7.38 $\pm 0.14 \mathrm{~Hz}$ in control to $2.76 \pm 0.14 \mathrm{~Hz}$ in mutant mice $p<0.0001$; Fig. 3.9B), whereas in both cases the amplitude reduction was almost the same about $29 \%$ $(68.97 \pm 0.89 \mathrm{pA}$ in controls to $48.72 \pm 1.03 \mathrm{pA}$; $\mathrm{p}<0.0001$; Fig. 3.9C; 3.6C).

\subsubsection{Miniature glycinergic postsynaptic currents in NL 2 deficient mice}

Next we analyzed the glycinergic minis in NL $2 \mathrm{KOs}$, adding $10 \mu \mathrm{M} \mathrm{CNQX}, 10 \mu \mathrm{M}$ AP5, $1 \mu \mathrm{M}$ bicuculline and $0.5 \mu \mathrm{M}$ tetrodoxin into extracellular solution. The comparison between GABAergic and glycinergic minis showed the following differences. The frequency of glycinergic mPSCs was decreased $60 \%(1.07 \pm$ $0.05 \mathrm{~Hz}$ in control to $0.4325 \pm 0.05 \mathrm{~Hz}$ in mutant cells; $p<0.0001$; Fig. 3.10B), while the frequency of GABAergic minis (Fig. 3.7B) was diminished $20 \%$ more. 
In mice lacking NL 2 the reduction of amplitude of glycinergic mPSCs compared to amplitude of miniature GABAergic postsynaptic currents (Fig. 3.7C) was $10 \%$ less $(55.56 \pm 1.21 \mathrm{pA}$ in control to $46.25 \pm 1.85 \mathrm{~Hz} \mathrm{KO}$ mice; $p<0.0001$; Fig. $3.10 \mathrm{C})$.

A WT

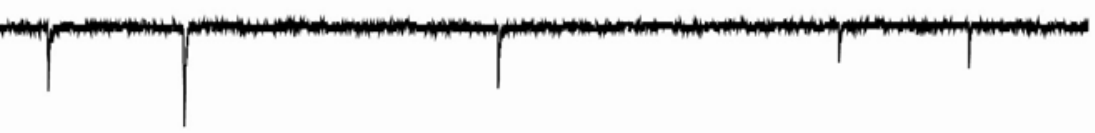

KO

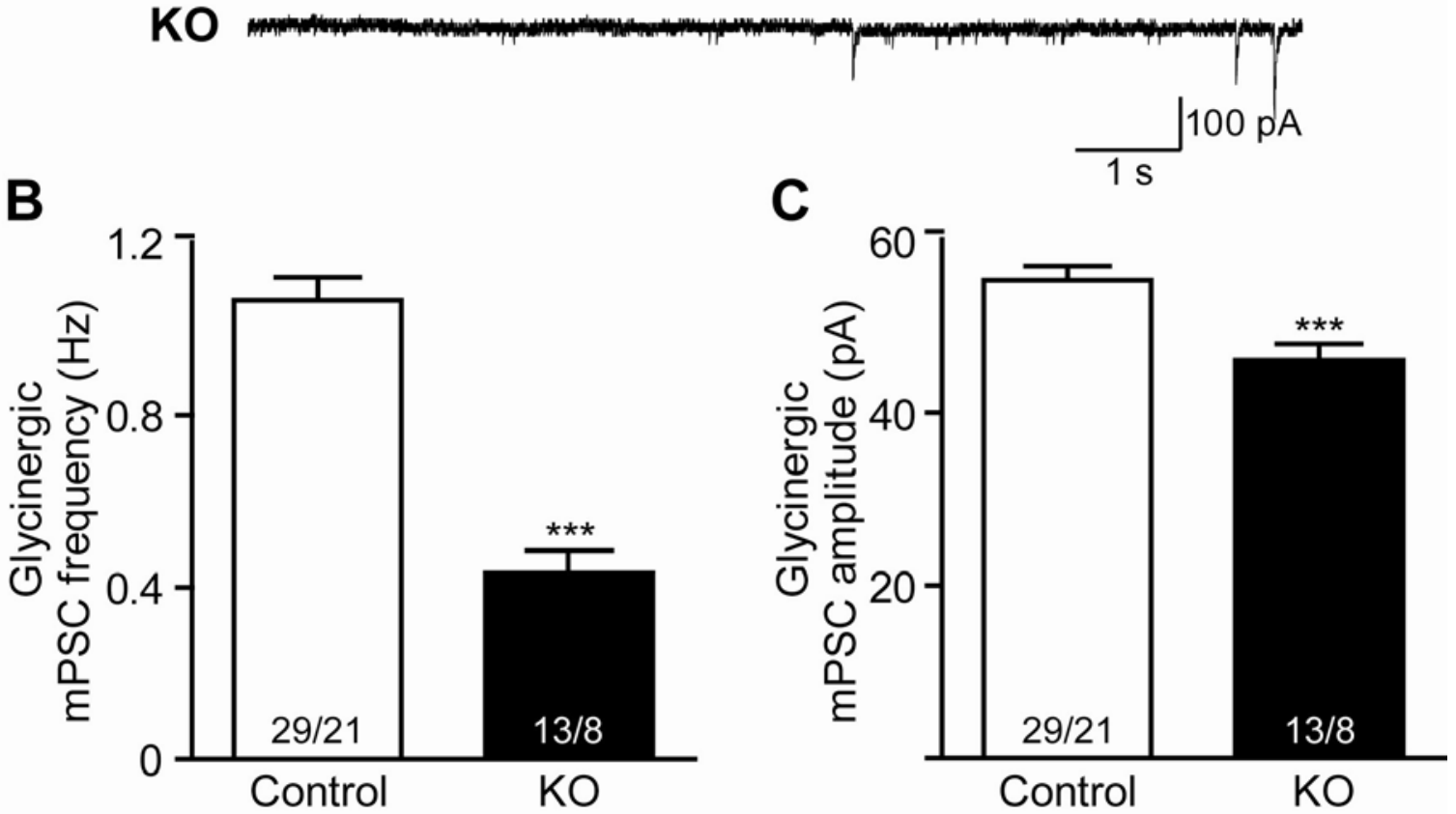

Figure 3.10 Decreased miniature glycinergic PSCs in PBC neurons of NL 2 KO mice.

(A) Sample traces of pharmacologically isolated miniature glycinergic postsynaptic currents in control and NL 2 deficient mice. Frequency (B) and amplitude (C) of glycinergic mPSCs are decreased less than frequency and amplitude of GABAergic minis. Numbers within bar graphs indicate number of cells and mice tested for each genotype. Error bars indicate SEM.

\subsubsection{Direct application of glycine to the postsynaptic neuron}

To test weather function of postsynaptic glycine receptors is depressed in mutant mice, we recorded the response to direct pressure application of glycine $(5 \mathrm{mM})$ with 500 mbar for $500 \mathrm{~ms}$ to the PBC neuron in the presence of $10 \mu \mathrm{M} \mathrm{CNQX}, 10$ $\mu \mathrm{M}$ AP5, $1 \mu \mathrm{M}$ bicuculline and $0.5 \mu \mathrm{M}$ tetrodoxin. Pressure ejection of glycine elicited different response in all genotypes (3.11A, B). The synaptic response to 
glycine was decreased about $40 \%$ from $6.15 \pm 0.25 \mathrm{nA}$ in control to $3.74 \pm 0.45$ $\mathrm{nA}$ in neuroligin 2 mutant mice; $(p<0.0001$; Fig.3.11B), whereas the synaptic response caused by direct application of $\mathrm{GABA}_{A}$ - receptor agonist muscimol was reduced by more than $60 \%$ (Fig. 3.8B).

Thus, these data revealed reduction of spontaneous and miniature glycinergic postsynaptic currents, but taken together all our experiments performed in brainstem PBC neurons indicate that deletion of $\mathrm{NL} 2$ impairs GABAergic synaptic transmission stronger, than glycinergic synaptic transmission.

A

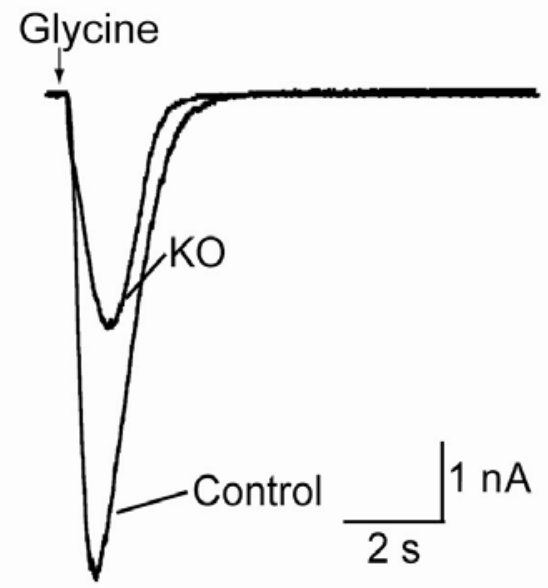

B

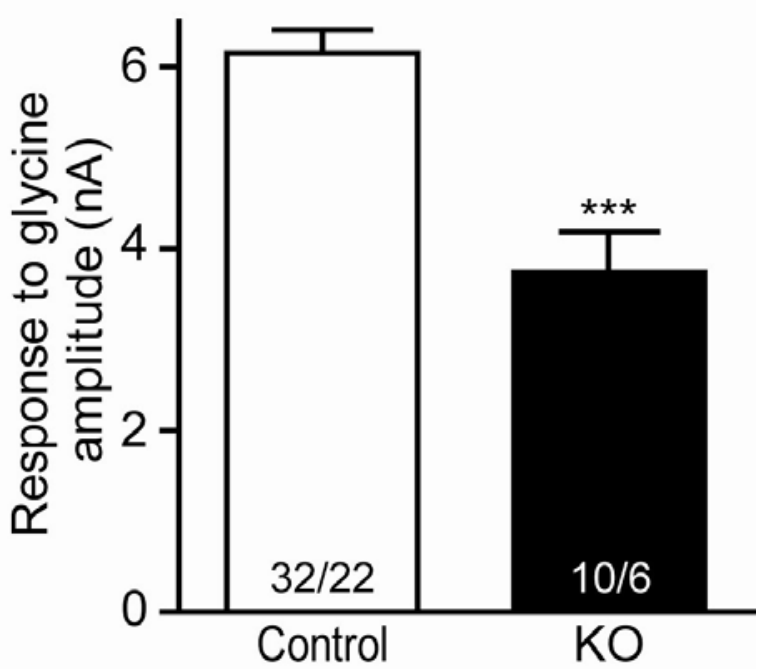

Figure 3.11 Direct pressure application of glycine elicits different responses in control and NL 2 KO neurons.

(A) Sample recordings and (B) synaptic responses in PBC neurons from littermate control and NL $2 \mathrm{KO}$ mice in presence of $0.5 \mu \mathrm{M}$ tetrodoxin, bicuculline, CNQX and AP5 after application of $5 \mathrm{mM}$ glycine. Numbers within bar graphs indicate number of cells and mice tested for each genotype. Error bars indicate SEM. 


\subsubsection{Unaltered excitatory synaptic transmission in PBC of mice lacking} NL 2

In many recent studies performed in neuronal cell cultures and brain tissues has been shown the role of different neuroligins in synaptogenesis and synaptic function. Despite some discrepant experimental results gotten by different research groups, there is a main outcome suggesting the potential role of $\mathrm{NL} 1$ at excitatory and for NL 2 at inhibitory synapses.

\subsubsection{Unaltered spontaneous excitatory postsynaptic currents in NL 2 KO mice}

Recordings of excitatory postsynaptic currents in preBötzinger complex of NL2 deficient mice showed completely different picture as compared to GABAergic/glycinergic synaptic transmission. Our experiments with NL 1- 3 triple $\mathrm{KO}$ mice revealed that deletion of neuroligins brings to significant changes in glutamatergic transmission, most probably evoked by lack of neuroligin 1 and 3 , but not by NL 2, which is concentrated at inhibitory synapses (Varoqueaux et al; 2004; Prange et al; 2004, Chih et al; 2005). As another functional evidence for this may serve our data obtained in acute brainstem slices from NL 2 KO mice, which show unchanged spontaneous and miniature excitatory postsynaptic currents performed in mice lacking NL 2. For this purpose we first examined spontaneous EPSCs in PBC neurons in presence of $1 \mu \mathrm{M}$ bicuculline and $1 \mu \mathrm{M}$ strychnine in extracellular solution to block GABAergic and gycinergic, synaptic transmission, respectively. Analyzes of spontaneous excitatory postsynaptic currents revealed no differences in frequency $(0.97 \pm 0.05 \mathrm{~Hz}$ in control to $0.87 \pm$ $0.07 \mathrm{~Hz}$ in mutant cells; non significant; Fig. 3.12B) and amplitude (32.81 \pm 0.49 $\mathrm{pA}$ in control to $31.43 \pm 0.6 \mathrm{pA}$ in NL $2 \mathrm{KO}$ mice; ns; Fig. 3.12C) between control and NL 2 deficient mice. 


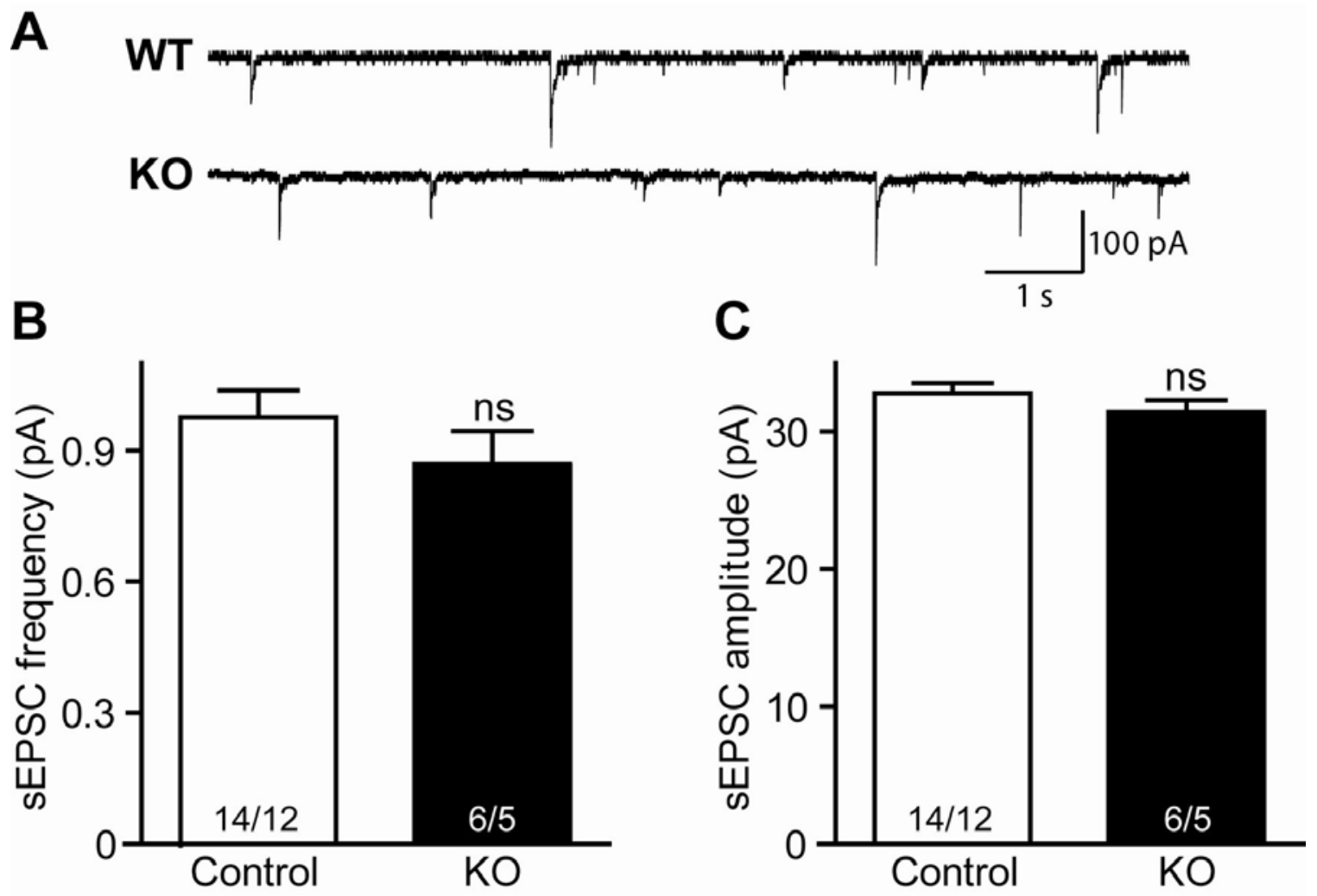

Figure 3.12 Deletion of NL 2 has no effect on spontaneous EPSCs.

Sample traces (A) of sEPSCs in control and NL $2 \mathrm{KO}$ mice in presence of $1 \mu \mathrm{M}$ bicuculline and $1 \mu \mathrm{M}$ strychnine. Frequency (B) and amplitude (C) of spontaneous excitatory postsynaptic currents remained unchanged in neuroligin 2 mutant mice compared to controls. Numbers within bar graphs indicate number of cells and mice tested for each genotype. Error bars indicate SEM.

\subsubsection{NL 2 deficient mice have unchanged spontaneous miniature EPSCs}

We next monitored pharmacologically isolated spontaneous excitatory postsynaptic currents, blocking action potential dependent network activity. Experiments were performed in brainstem PBC neurons in presence of $1 \mu \mathrm{M}$ bicuculline, $1 \mu \mathrm{M}$ strychnine and $0.5 \mu \mathrm{M}$ tetrodoxin in extracellular solution. The frequency of mEPSCs was identical with $0.77 \pm 0.07 \mathrm{~Hz}$ in control and $0.77 \pm$ $0.08 \mathrm{~Hz}$ in NL 2 deficient mice (ns; Fig. 3.13B). The amplitude of mEPSCs was slightly increased from $34.97 \pm 0.83 \mathrm{pA}$ in control and $37.62 \pm 0.81 \mathrm{pA}$ in mutant mice (ns; Fig. 3.13C). 
A

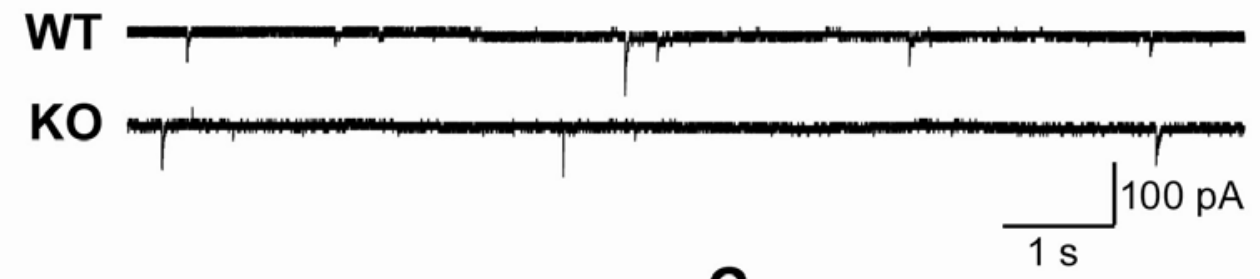

B

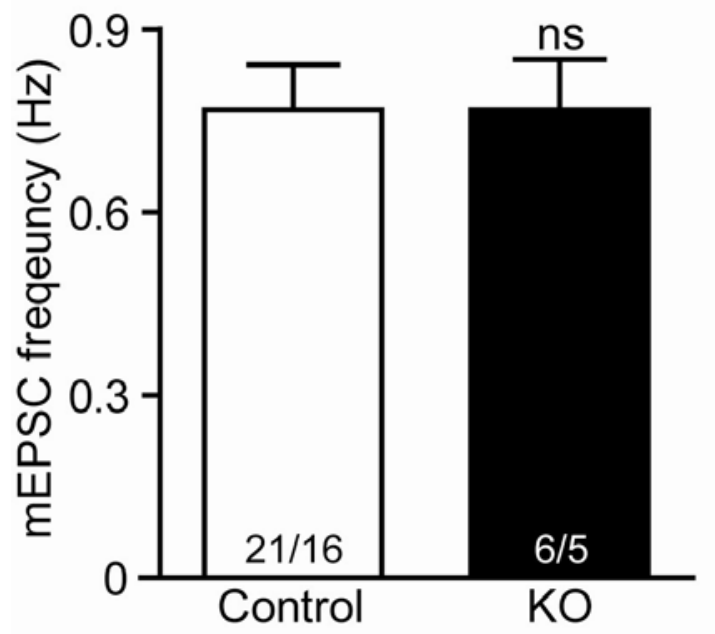

C

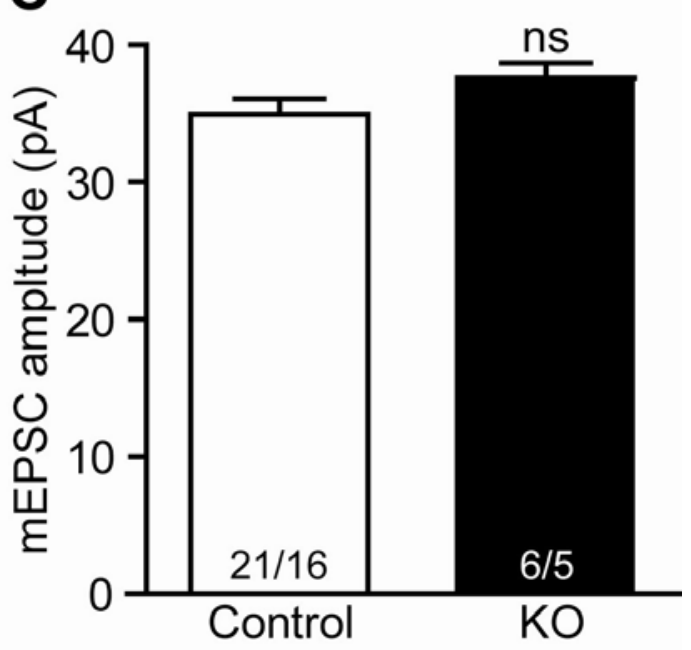

Figure 3.13 Unaltered spontaneous miniature excitatory postsynaptic currents in brainstem neurons from NL 2 KO mice.

Representative traces (A), frequency (B) and amplitude (C) of spontaneous miniature excitatory postsynaptic currents in control and mutant mice. Numbers within bar graphs indicate number of cells and mice tested for each genotype. Error bars indicate SEM.

\subsubsection{The effect of pressure applied glutamate}

In this series of experiments we recorded the response to extracelluar pressure applied gluatamate $(5 \mathrm{mM})$. The experiments were performed in presence of $1 \mu \mathrm{M}$ bicuculline, $1 \mu \mathrm{M}$ strychnine and $0.5 \mu \mathrm{M}$ tetrodoxin, which were applied into bath solution. Direct ejection of glutamate $(500 \mathrm{mbar}, 500 \mathrm{~ms})$ to the postsynaptic $\mathrm{PBC}$ neuron in contrast to responses evoked by muscimol or glycine application (Fig. 3.8A, B; Fig.3.11A, B), did not provoke decrease of synaptic response in NL $2 \mathrm{KO}$ mice; rather the response to glutamate in NL2 mutant mice was increased from $1.42 \pm 0.28 \mathrm{nA}$ in control to $2.29 \pm 0.49 \mathrm{nA}$ in NL 2 deficient mice (ns; Fig. 3.14B). 
A

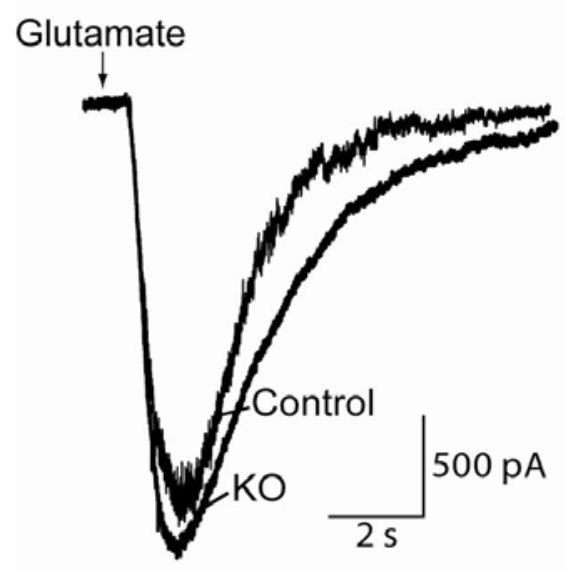

B

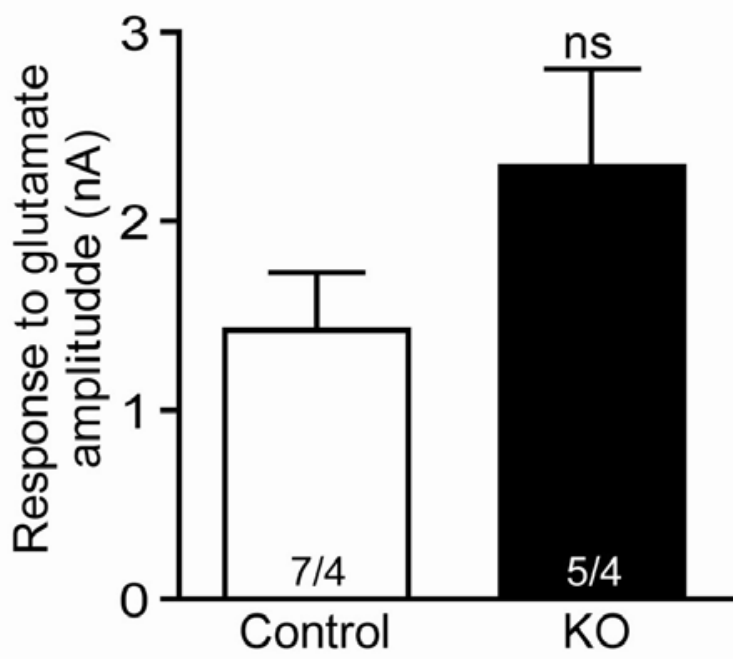

Figure 3.14 The responses elicited by pressure application of glutamate.

Sample recordings (A) and amplitude (B) of response to pressure applied glycine $(5 \mathrm{mM})$ in control and NL 2 KO mice in presence of bicuculline, AP5 and tetrodoxin. Numbers within bar graphs indicate number of cells and mice tested for each genotype. Error bars indicate SEM.

\subsection{Evoked neurotransmitter release in NH of NL 2 deficient mice}

After our observation that GABAergic/glycinergic synaptic transmission in brainstem PBC neurons is strongly impaired and glutamatergic transmissions is not affected in mice lacking NL 2, we next examined electrically evoked synaptic responses in completely different group of respiratory brainstem neurons. These experiments were performed stimulating the neurons in the vicinity of the preBötzinger complex and measuring the synaptic transmission in hypoglossal motor neurons by whole-cell patch-clamp recordings. The distance between stimulation and patch electrodes was kept similar on all slices. For these recordings the highest stimulation strength $(10 \mathrm{~mA})$ was used. 


\subsubsection{Lack of neuroligin 2 slightly decreases electrically evoked synaptic transmission in hypoglossal neurons}

To analyze evoked neurotransmission in $\mathrm{NH}$, first we recorded evoked postsynaptic currents. In contrast to synaptic transmission measured in brainstem PBC neurons, electrically evoked neurotransmission in hypoglossal neurons was less decreased. The amplitude of evoked synaptic responses was reduced from $491.3 \pm 105.9 \mathrm{pA}$ in control to $300.9 \pm 70.04 \mathrm{pA}$ in NL 2 mutant mice was (ns; Fig. 3.15B).

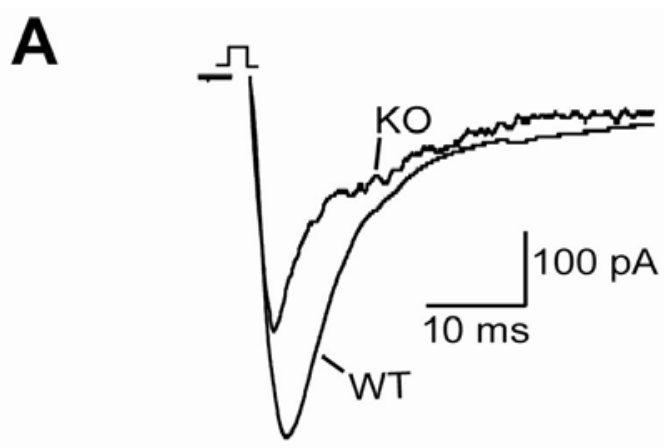

B

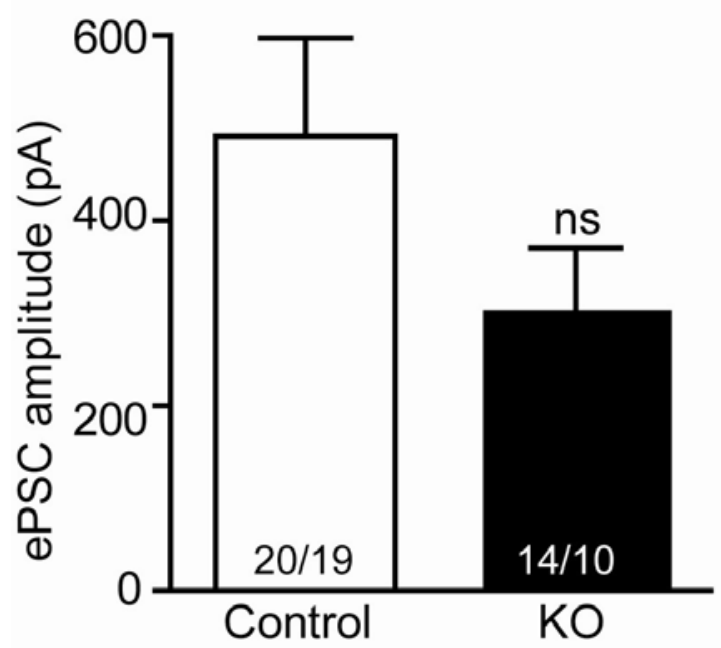

C

Figure 3.15 Electrically evoked synaptic responses in brainstem hypoglossal neurons.

(A) Sample recordings of ePSCs in hypoglossal neurons from control and neuroligin $2 \mathrm{KO}$ cells during field stimulation of axons from preBötzinger complex. Amplitude (B) of ePSCs is decreased in $\mathrm{KO}$ as compared to control mice. Deletion of NL 2 significantly increases the failure rate $(C)$ of ePSCs. Numbers within bar graphs indicate number of cells and mice tested for each genotype. Error bars indicate SEM. 
Despite the fact that we used supramaximal stimulation $(10 \mathrm{~mA})$, the failure rate of evoked postsynaptic currents was increased in NL $2 \mathrm{KO}$ neurons as compared to controls $(0.2 \% \pm 0.2 \%$ in control to $6.57 \% \pm 3.67 \%$ in NL2 mutant mice; p<0.01; Fig. 3.15C).

\subsubsection{Electrically evoked inhibitory neurotransmission is affected in neuroligin 2 mutant mice}

Recordings of evoked inhibitory postsynaptic transmission were performed in the $\mathrm{NH}$ in presence of $10 \mu \mathrm{M} C N Q X$ and $10 \mu \mathrm{M}$ AP 5. Deletion of NL 2 caused $57 \%$ reduction in the amplitude of elPSCs from $94.70 \pm 15.63$ pA in control mice to $40.56 \pm 15.57 \mathrm{pA}$ in KOs ( $\mathrm{p}<0.01$; Fig. 3.16B). Similar to recordings of evoked PSCs in $\mathrm{NH}$, for these experiments also maximal stimulation length (10 $\mathrm{mA})$ was used. The failure rate was markedly diminished in neuroligin 2 mutant mice (1.66 $\% \pm 1.04 \%$ in control to $29.60 \% \pm 19.46 \%$ in NL 2 KO cells; $p<0.01$; Fig. $3.16 \mathrm{C}$ ). 


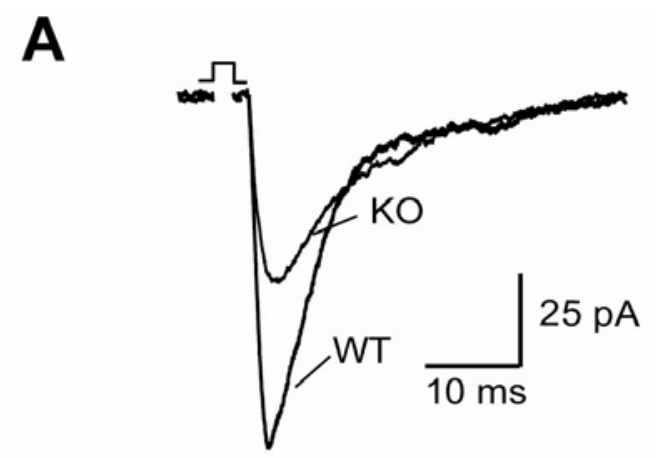

B

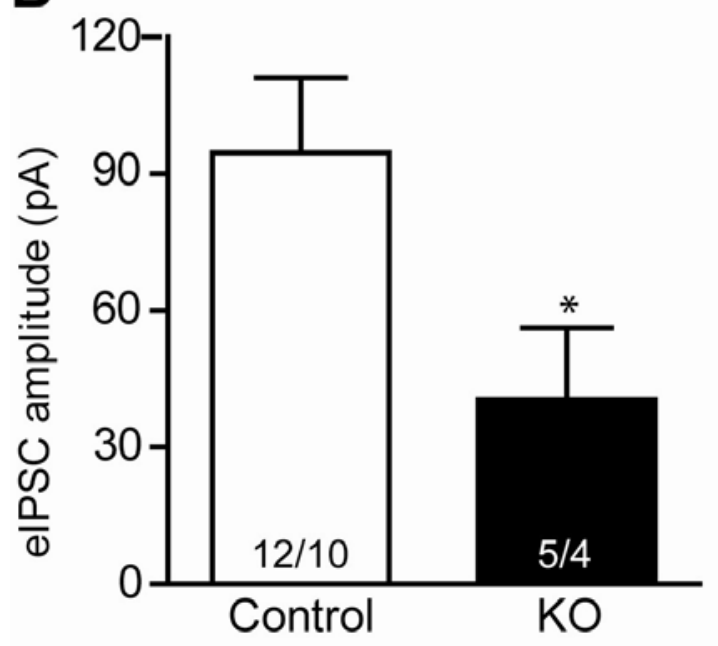

C

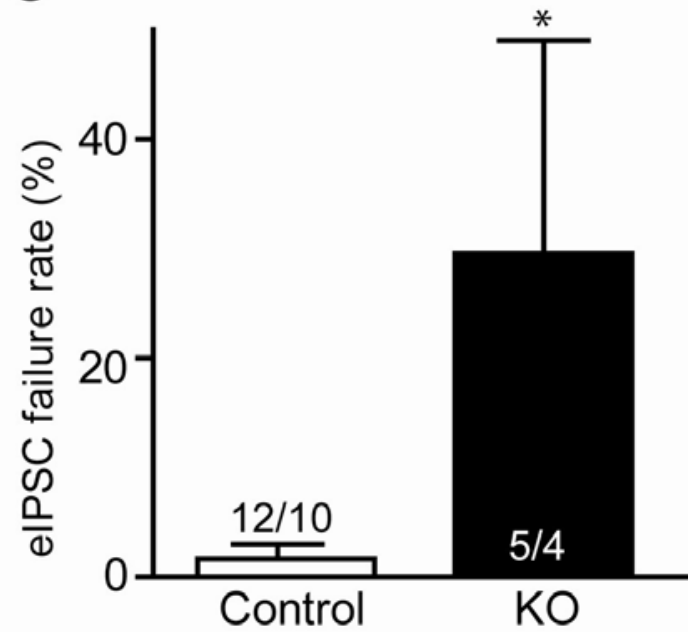

Figure 3.16 Decreased evoked inhibitory neurotransmission in NH in NL $2 \mathrm{KO}$ mice.

(A) Sample traces of pharmacologically isolated evoked GABAergic/glycinergic postsynaptic currents monitored by whole-cell recordings in hypoglossal neurons. Diminished amplitude (B) and increased failure rate $(C)$ of elPSCs in NL 2 KO compared to control mice. Numbers within bar graphs indicate number of cells and mice tested for each genotype. Error bars indicate SEM.

These results show that the lack of NL 2 leads to stronger impairment of inhibitory synaptic transmission in the preBötzinger complex, than in hypoglossal motor neurons. Nevertheless, deletion of NL 2 impairs the GABAergi/glycinergic synaptic function in neuronal network of respiratory brainstem. 


\subsubsection{Evoked EPSCs is not different between control and NL 2 KO mice}

Finally, we examined electrically evoked glutamatergic synaptic transmission in respiratory brainstem. Recordings were done in hypoglossal motor neurons of littermate control and NL 2 mutant mice, having a $1 \mu \mathrm{M}$ bicuculline and $1 \mu \mathrm{M}$ strychnine in extracellular solution, in order to block inhibitory synaptic transmission. Analyzing the amplitude of electrically evoked synaptic responses, we detected almost no change between control and NL 2 mutant mice (453.7 \pm 116.3 pA in control to $392.8 \pm 90.3 \mathrm{pA}$; ns; Fig. 3.17B), which means that excitatory synapses in respiratory brainstem are functioning normally. The very small failure rate was similar in all tested genotypes $(0.33 \% \pm 0.33 \%$ in control to $0.31 \% \pm 0.31 \%$ in NL $2 \mathrm{KO}$ mice; ns; Fig.3.17C). These data are in agreement with results obtained from PBC neurons that show no change in amplitude of spontaneous and miniature glutamatergic postsynaptic currents in control and NL 2 knockout mice. 

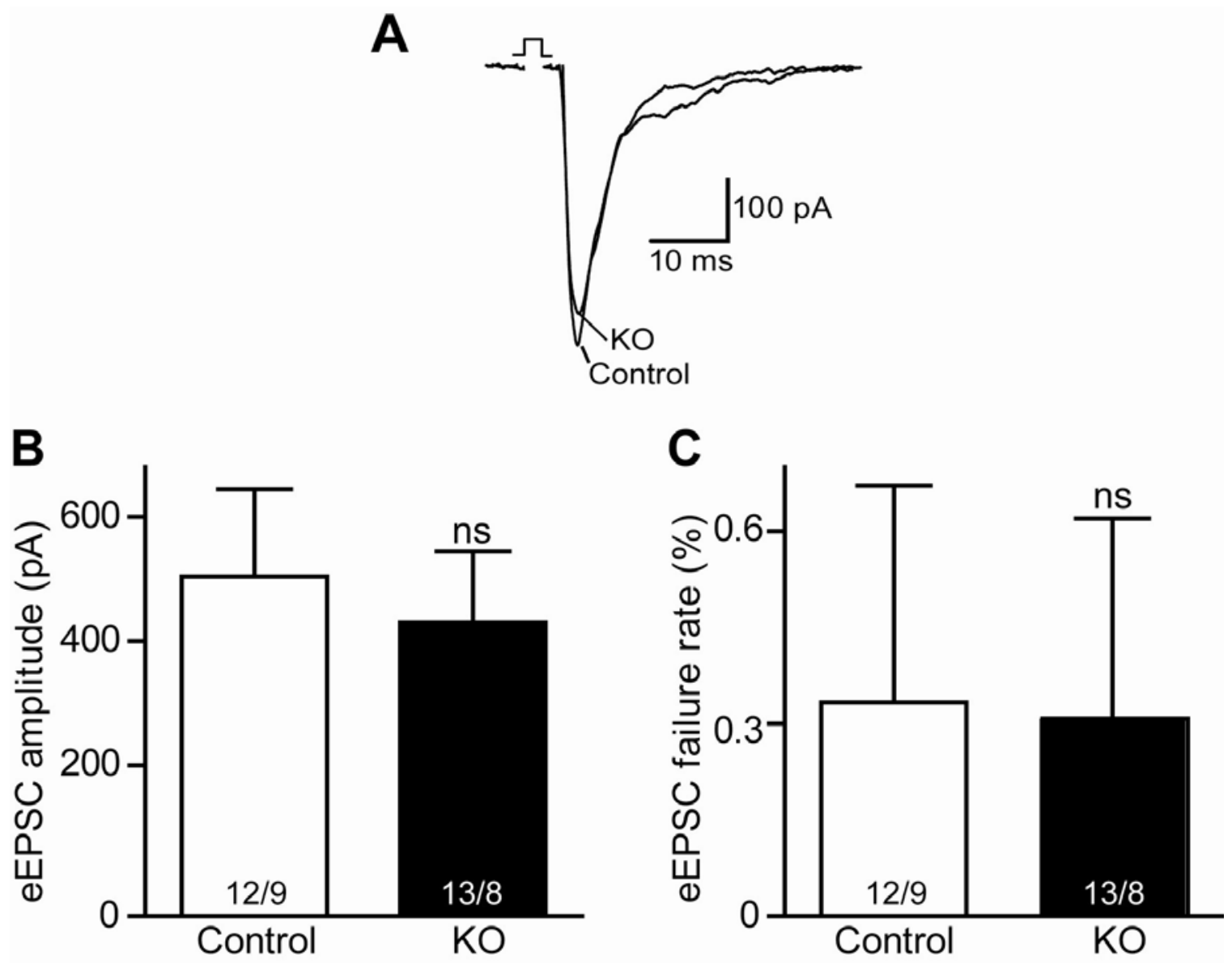

Figure 3.17 Unaltered evoked excitatory neurotransmission in NH of NL 2 KO mice.

(A) Sample recordings of pharmacologically isolated evoked excitatory postsynaptic currents (eEPSCs) in brainstem hypoglossal neurons from littermate control and NL 2 KO mice. Unchanged amplitude (B) and failure rate (C) of eEPSCs in hypoglossal neurons from control and NL 2 mutant mice in response to extracellular stimuli. Numbers within bar graphs indicate number of cells and mice tested for each genotype. Error bars indicate SEM. 


\subsection{Synaptic protein expression and synaptogenesis in brainstem of NL 2 KO mice}

\subsubsection{The expression of synaptic proteins in brainstem of NL $2 \mathrm{KO}$ mice}

To determine the consequences of NL 2 deletion on the expression of different synaptic proteins in brainstem we performed Western blot analyses using brainstem homogenates of littermate control and NL 2 mutant mice at postnatal day 5. The expression of all tested general (synaptophysin, as presynaptic marker), excitatory (VGluT 1/2, PSD-95) and inhibitory (VIAAT, gephyrin) synaptic markers were similar in littermate control and neuroligin $2 \mathrm{KO}$ mice (Fig.3.18). Likewise, the expression levels of inhibitory (GABAaR a1, GABAaR b2/3, GABAaR g2, GlycR) and excitatory synaptic markers (NMDAR 1, GluR 1 and GluR 2/3) were unchanged in mice lacking NL 2 as determined by Western blot analyses (Fig. 3.18).

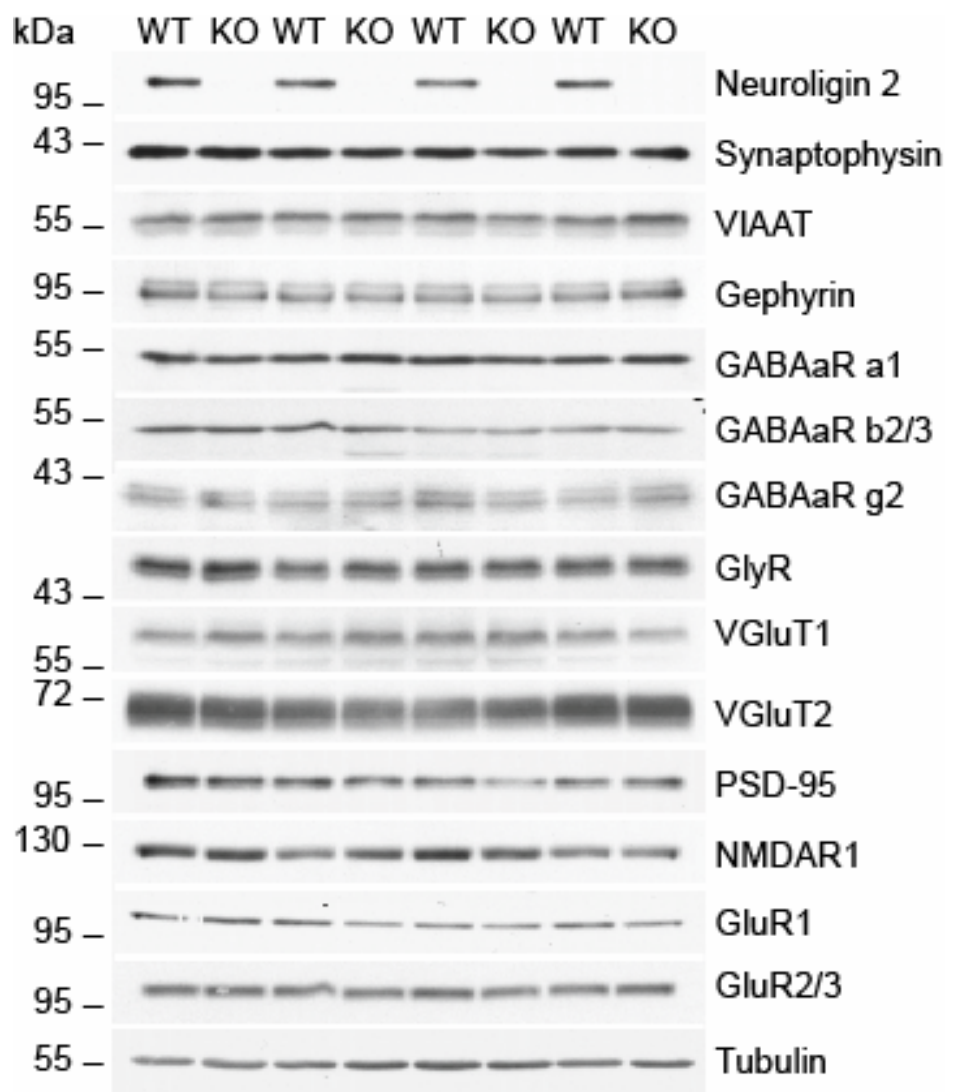

Figure 3.18 Unaltered synaptic proteins expression in NL $2 \mathrm{KO}$ mice.

Expression patterns of inhibitory (VIAAT, Gephyrin, GABAaR a1, GABAaR b2/3, GABAaR g2, GlycR) and excitatory (VGlut2, PSD-95, NMDAR 1, GluR 1, GluR 2/3) synaptic markers as determined by Western blotting of control (WT) and $\mathrm{NL} 2 \mathrm{KO}$ (KO) mice brainstem homogenates at postnatal day 5 . Tubulin is used as a loading control. 
The finding that the loss of neuroligin 2 does not cause changes in the expression level of excitatory and inhibitory synaptic markers is in agreement with data obtained from morphological experiments (see below).

\subsubsection{Synaptogenesis in respiratory brainstem of NL $2 \mathrm{KO}$ mice}

\subsubsection{Unaltered GABAergic/glcinergic synapse numbers in preBötzinger complex of NL 2 KO mice}

As described above, our electrophysiological experiments revealed strong impairment of inhibitory synaptic activity in respiratory brainstem of NL $2 \mathrm{KO}$ mice and to examine whether this functional change in mutant animals is due to aberrant synaptogenesis, we performed immunofluorescence double labeling in preBötzinger complex of control and neuroligin $2 \mathrm{KO}$ mice at the postnatal day 5 . In order to determine the number of matched and mismatched pre- and postsynaptic GABAergic/glycinergic specializations in control and NL $2 \mathrm{KO}$ mice, the brain sections containing preBötzinger complex were costained for VIAAT (marker for inhibitory presynapses) and gephyrin (inhibitory postsynaptic marker) (Fig.3.19A).Quantification of isolated VIAAT-labeled immunoreactive puncta revealed almost similar number of GABAergic/glycinergic presynaptic structures in control and NL2 KO PBC $(20.03 \pm 2.09$ in control mice to $21.31 \pm 4.26$ in KOs; ns; Fig.3.19B). The number of GABAergic/glycinergic presynaptic postsynaptic structures was decreased about $27 \%$ from $33.8 \pm 5.75$ in control to $24.52 \pm 2.49$ in NL 2 KO PBC, (ns; Fig.3.19B), which was not statistically significant. Quantification of colocalized puncta showed that the number of GABAergic/glycinergic synapses is identical in preBötzinger complex of control and NL $2 \mathrm{KO}$ mice $(11.1 \pm 1.66$ in control mice to $10.9 \pm 2.2$ in KOs; ns; Fig.3.19B). Moreover, analyzing the staining intensity we did not observe any difference between control mice and NL 2 KOs.

These experiments indicate that in the absence of neuroligin 2 the GABAergic/glycinergic synapse numbers remain unchanged. 
A
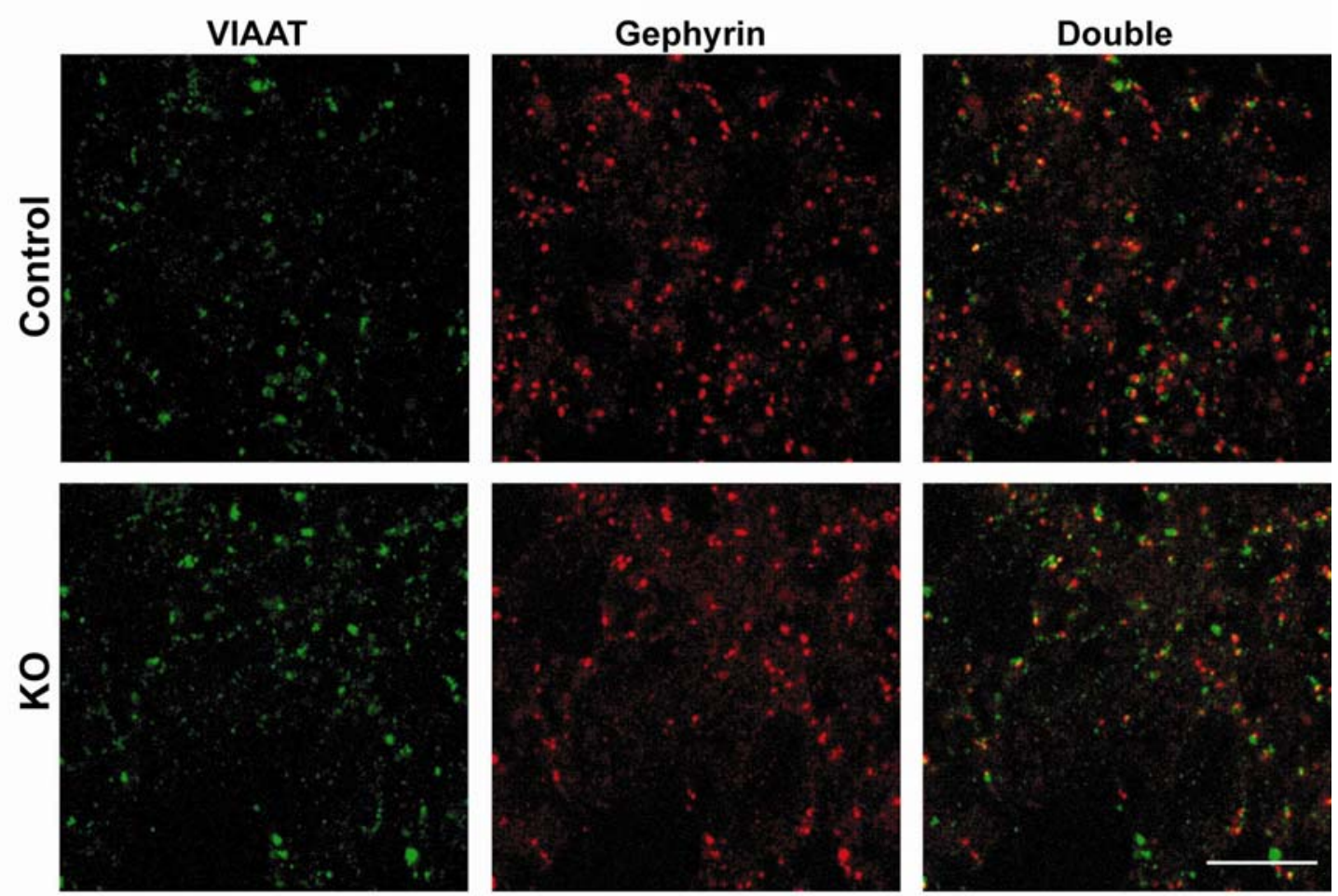

B

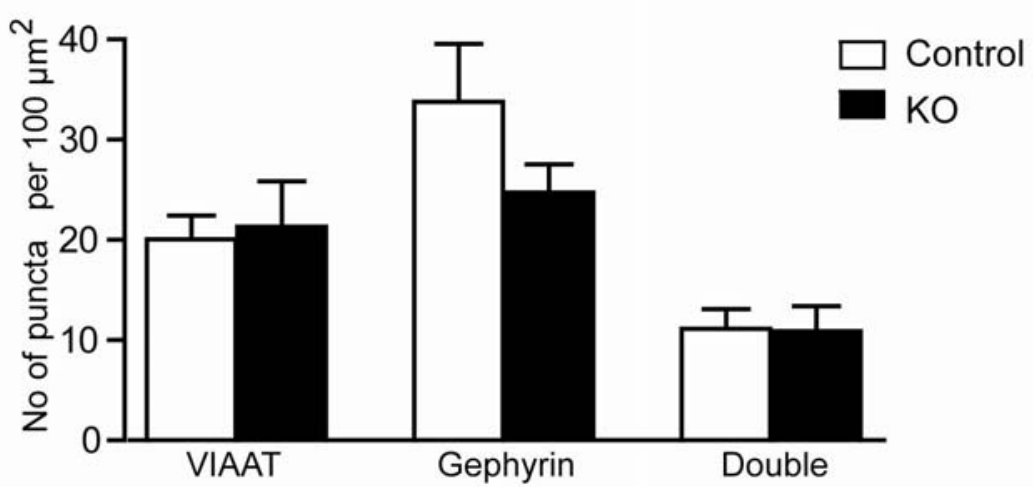

Figure 3.19 Inhibitory pre- and postsynaptic specializations in PBC neurons.

(A) Representative micrographs of the PBC area of control (Control, $n=5)$ and NL $2 \mathrm{KO}(\mathrm{KO}, \mathrm{N}=5)$ sections after double labeling for inhibitory presynapses (stained for VIAAT, green) and inhibitory postsynapses (stained for gephyrin, red). (B) The quantification of isolated and colocalized VIAAT and gephyrin puncta in the PBC of control (Control, white) and NL 2 KO mice (KO, black). Scale bars, $10 \mu \mathrm{m}$. Error bars indicate SEM. 


\subsubsection{Deletion of NL 2 leads to the slight increase of glutamatergic synaptic specializations}

In a final series of experiments, we performed double immunofluorescence staining for glutamatergic specializations in control and NL $2 \mathrm{KO}$ preBötzinger complex in order to examine the role of $\mathrm{NL} 2$ on glutamatergic pre- and postsynaptic compartments. The brain sections containing PBC were colabeled for VGluT 1/2 (markers for glutamatergic presynapses) and ProSAP1 (glutamatergic postsynaptic marker) (Fig.3.20A). Quantification of isolated VGluT 1/2 puncta and ProSAP 1 puncta in PBC revealed no significant changes in the number of pre- and postsynaptic compartments. The number of pre- and postsynaptic structures was slightly increased in NL $2 \mathrm{KO}$ mice as compared to controls from $22.29 \pm 3.35$ in control to $32.33 \pm 6.23$ in KO PBC and from $36.98 \pm$ 4.88 in control to $43.57 \pm 6.09$ in NL $2 \mathrm{KO}$ mice (ns; Fig.3.20B), respectively. Likewise, the deletion of NL 2 caused a slight increase of glutamatergic synapse numbers, which was not statistically significant. The staining intensity was similar in all tested genotypes. Analyzing the ratio between the number of glutamatergic terminals as assessed by VGluT1/2 immunoreactivity and the number of GABAergic/glycinergic terminals as assessed by VIAAT immunoreactivity, we found that it was increased in the PBC of NL $2 \mathrm{KO}$ mice, which was not statistically significant (3.21). This alteration could occur due to small changes in the absolute numbers of glutamatergic and GABAergic/glycinergic synapses. These morphological data obtained from respiratory brainstem of NL $2 \mathrm{KO}$ mice show again that neuroligins are not essential for synapse formation. 
A
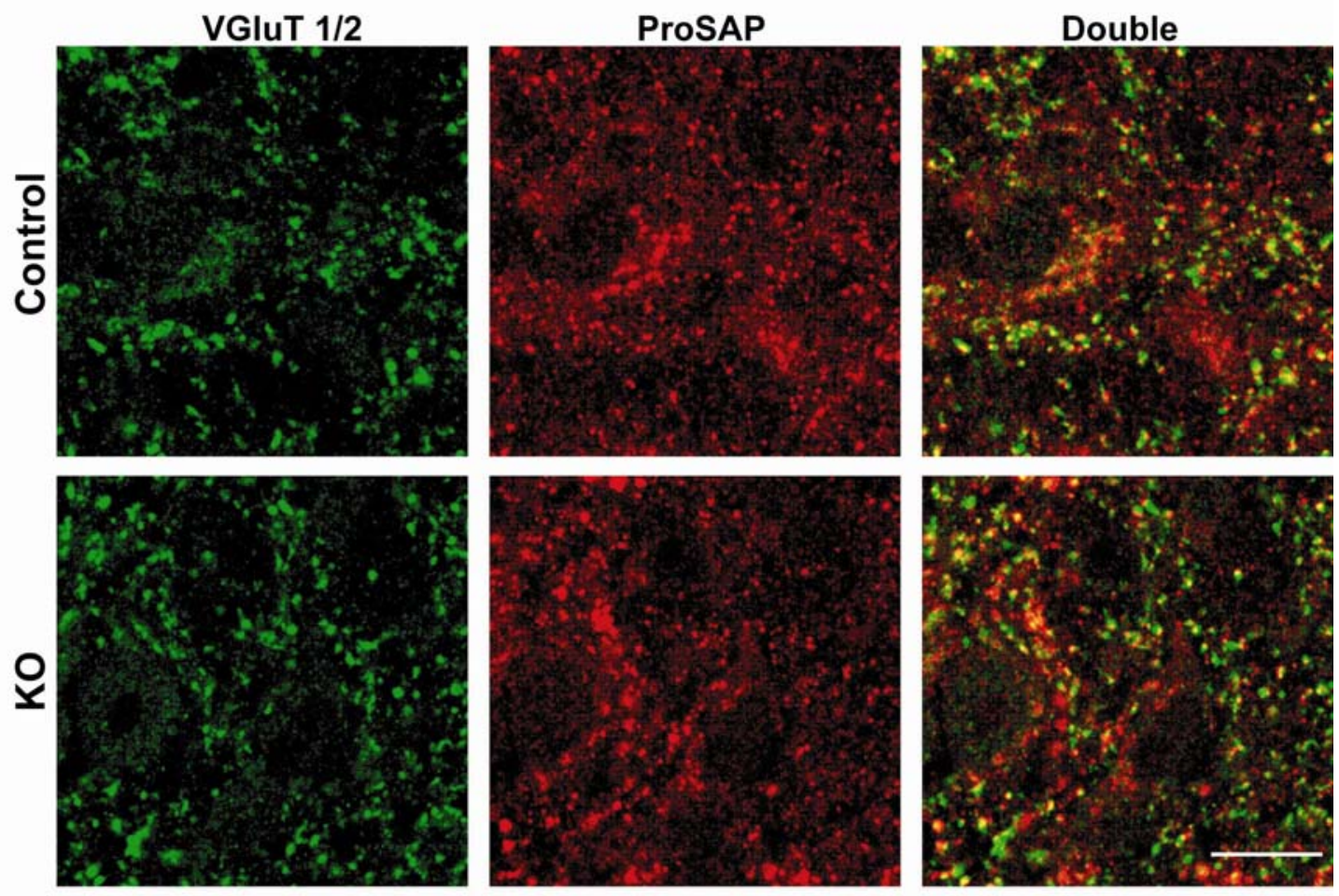

B

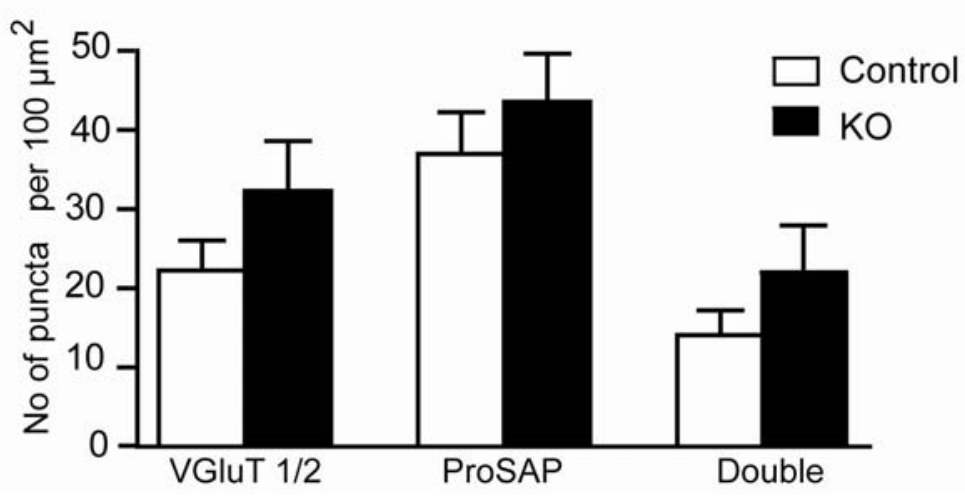

Figure 3.20 Glutamatergic pre- and postsynaptic specializations in PBC neurons.

(A) Representative micrographs of the PBC area of control (Control, $n=5)$ and NL $2 \mathrm{KO}(\mathrm{KO}, \mathrm{N}=5$ ) sections after double labeling for glutamatergic presynapses (stained for VGluT1/2, green) and glutamatergic postsynapses (stained for ProSAP, red). (B) The quantification of isolated and colocalized VGluT1/2 and ProSAP puncta in the PBC of control (Control, white) and NL 2 KO mice (KO, black). Scale bars, $10 \mu \mathrm{m}$. Error bars indicate SEM. 


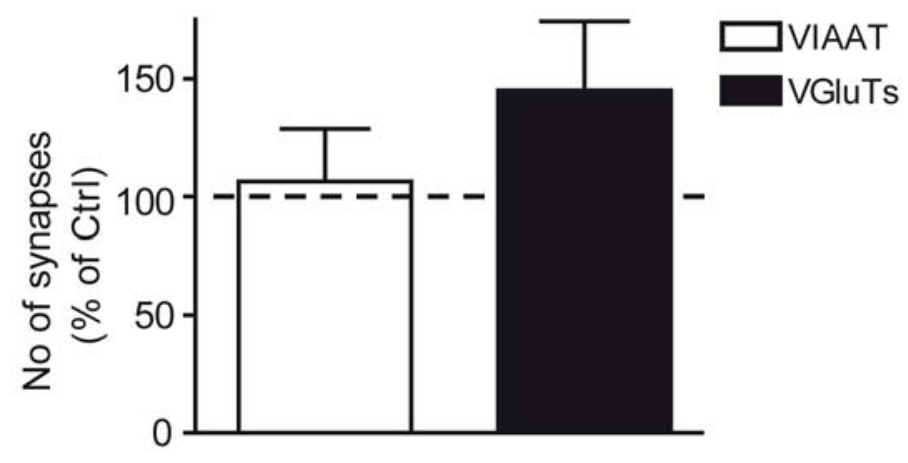

Figure 3.21 Altered ratio of glutamatergic versus GABAergic/glycinergic terminals in the preBötzinger complex of NL 2 KO mice Number of GABAergic/glycinergic (white) and glutamatergic (black) synapses in the PBC of NL $2 \mathrm{KO}$ mice plotted as the percentages of glutamatergic and GABAergic/glycinergic synapses observed in the control (Ctrl). 


\section{Discussion}

Neuroligins are present at postsynaptic terminal and together with their presynaptic $\alpha$-and $\beta$-neurexin binding partners form asymmetric intercellular junctions. Several data obtained from cultured neurons suggest the essential role of neuroligins in synapse formation and synaptic function (Chih et al., 2005; Dean et al., 2003; Graf et al., 2004; Levinson et al., 2005; Nam and Chen, 2005; Prange et al., 2004; Scheiffele et al., 2000). In this present project to determine the role of neuroligins in synaptogenesis and synapse function in vivo, we used neuroligin triple knockout mice (NL 1-3 TKO). For detailed investigation of inhibitory synaptic activity, mice lacking neuroligin 2 (NL 2 KO) were used.

\subsection{Neuroligin triple knockout mice}

$\mathrm{NL}$ triple KO mice have impaired postnatal survival. They die after birth within few hours due to respiratory problems, whereas all single and double KOs are viable. Neuroligin 1-3 triple KOs display irregular and flat breathing movements as determined by whole-body pletismography. They have slightly reduced body weight as compared to single and double KO littermates.

The present data obtained from respiratory brainstem of neuroligin triple KO mice show that all isoforms of neuroligins are not essential for formation of initial synaptic contacts and play an important role in synaptic function. The deletion of all three neuroligins leads to severe decrease in spontaneous inhibitory activity and moderate reduction in spontaneous excitatory activity in the respiratory brainstem, which causes respiratory failure.

\subsubsection{Synaptogenesis in mice lacking NLs 1-3}

Our detailed morphometric analyses of synapse types and densities at light microscopic and ultrastructural level in hippocampus and neocortical cultured neurons, and respiratory brainstem slices derived from NL 1-3 triple KO mice did not reveal the significant role of neuroligins in initial formation of synaptic contacts. The immunoflourescense double staining of the synaptic markers 
synapsin and PSD-95 showed the same synapse densities in control and neuroligin 1-3 triple KO hyppocampal cultured neurons. In addition, the ultrastructural studies of neuron cultures from control and neuroligin triple KO cortex demonstrated no abnormalities in synaptic structure. Different synaptic parameters such as a length of the postsynaptic density (PSD), width of the synaptic cleft and the number of docked vesicles were similar in control and neuroligin 1-3 triple $\mathrm{KO}$ mice. In vivo ultrastructural analyses of $\mathrm{PBC}$ containing respiratory brainstem slices showed similar numbers of synaptic specializations in NL 1-3 triple KO and control samples. Likewise, the number of pre- and postsynaptically double labeled glutamatergic and GABAergic/glycinergic synapses was almost identical in mutant and control mice. Interestingly, the ratio between the number of glutamatergic synapses and GABAergic/glycinergic synapses was increased in NL 1-3 triple KO mice, which was caused by only small and statistically insignificant changes in absolute GABAergic/glycinergic (decrease) and glutamatergic synapse numbers in the range of $15 \%$. The imbalance between excitation and inhibition caused by loss of neuroligins supports the hypothesis that neuroligins contribute to the homeostatic mechanism that maintains the E/l balance. The only significant change in NL 1-3 triple $\mathrm{KO}$ mice that we have observed was $30 \%$ reduction in the number of $G A B A_{A} R \alpha 1$ containing postsynaptic clusters. These findings are not in agreement with the effects of neuroligin RNAi knock-down in cultured neurons (Chih et al., 2005), where it is shown that the knock-down of even single neuroligin leads to the strong decrease in synapse numbers of cultured neurons in vitro, but are in line with observation obtained from a-neurexin triple KO mice (Missler et al., 2003), which revealed that the deletion of all three $\alpha$-neurexins has little effect on synapse densities. We propose that such a discrepancy between our data on NL 1-3 triple KOs and the data obtained after RNAi knockdown of neuroligins in cultured neurons (Chih et al., 2005) can be explained by activity-dependent homeostatic effect on synaptogenesis (Turigiaano and Nelson, 2004) or it is possible that off-target effects of the RNAi constructs cause perturbations of synapse stability. An alternative explanation for this discrepancy 
could be that differences between our data obtained from NL 1-3 triple KO mice and the published cell culture experiments on NLs are due to specific compensatory effects in the NL 1-3 triple KOs in vivo.

Thus, the present study shows that even the absence of all three neuroligins is not essential for initial synapse formation and neuroligins determine functional parameters of synapses by regulating the recruitment of synaptic proteins (e.g., $\mathrm{GABA}_{A}$ receptors).

\subsubsection{The role of NLs 1-3 in synaptic function}

\subsubsection{Severe impairment of inhibitory synaptic transmission in neuroligin triple KO mice}

The present study shows how compulsory are all three neuroligins together in synaptic function and the most obvious phenotypic characteristic of this is the early postnatal death of neuroligin triple $\mathrm{KO}$ mice. Because of the necessity to produce large numbers of NL triple knockout mice, we were forced to use NL 1 and NL 3 single and NL $1 / 3$ double $\mathrm{KO}$ mice as littermate controls in electrophysiological analyses in NL 1-3 triple KO mice. Our investigations revealed dramatic impairment of inhibitory synaptic transmission. Spontaneous GABAergic/glycinergic sPSC amplitudes in the preBötzinger complex and evoked GABAergic/glycinergic ePSC amplitudes in the $\mathrm{NH}$ were both strongly reduced in NL 1-3 triple KO mice. The frequency of spontaneous inhibitory PSCs in PBC neurons was also significantly decreased in triple knockout mice. Together with finding that the numbers of postsynaptic clusters containing $G_{A B A} R \alpha 1$ were reduced, and the numbers of inhibitory synapses were unchanged in the brainstem of NL 1-3 triple KO mice, these data indicate that initial formation of GABAergic synaptic contacts does not depend on neuroligins, but the absence of NLs leads to their dysfunction, possibly due to a deficiency of recruitment of receptors to GABAergic synapses. Interestingly, GABAergic/glycinergic mPSC frequencies in PBC were decreased, whereas MPSC amplitudes remained 
unaltered in mice lacking NL 1-3, suggesting that this functional shut-down may be caused by postsynaptic changes and presynaptic dysfunction. These results are in line with recent electrophysiological data obtained from RNAi knock-down experiments performed in cultured hippocampal neurons, where a strong reduction of NLs 1-3 expression decreases the frequency and amplitude of mIPSCs (Chih et al., 2005).

\subsubsection{Lack of NLs alters excitatory synaptic activity}

As known NL 1 (Song et al., 1999, Chih et al., 2006), NL 3 are preferentially localized to glutamatergic synapses and NL 2 is localized to inhibitory synapses (Varoqueaux et al., 2004), but interestingly the loss of neuroligins seem to affect inhibitory synaptic transmission more strongly than excitatory transmission. What could be the explanation of this? A biological reason for a preferential effect of the NL 1-3 triple KO on inhibitory synapses could be explained by the fact that glutamatergic synapses, in contrast to GABAergic/glycinergic synapses, contain multiple transsynaptic cell adhesion and signaling systems that interact with intracellular proteins (Garner et al., 2002; Kim and Sheng, 2004; Li and Sheng, 2003; Montgomery et al., 2004; Scheiffelle et al., 2003; Yamagata et al., 2003). The data obtained from excitatory synaptic transmission in brainstem revealed significant functional changes in mice that had deletion of NLs 1-3. Our analyses of spontaneous excitatory synaptic transmission showed reduction of frequency of sEPSCs in the preBötzinger complex of NL 1-3 triple KOs, while sEPSC amplitudes were similar in all NL KO combinations. The slight decrease of evoked excitatory ePSC amplitudes in hypoglossal neurons of NL 1-3 triple KOs was not statistically significant. Analyzing the miniature glutamatergic PSCs we found strongly reduced mPSC frequency in $\mathrm{PBC}$ neurons of neuroligin 1-3 triple $\mathrm{KO}$ mice and almost identical mPSC amplitudes in all NL KO combinations. By contrast, electrophysiological data obtained in cultured neocortical neurons showed no differences in AMPA mEPSCs between control and neuroligin triple $\mathrm{KO}$ neurons. In addition, the electrophysiological studies in triple-neuroligin 
knock-down hyppocampal neurons showed that the strong reduction of NLs 1-3 expression has little effect on mEPSC frequency and amplitude (Chih et al., 2005). Such a discrepancy between cultured neurons and acute slices indicate that these changes induced by a lack of neuroligins can be obscured in cultured neurons. These data, together with findings on brainstem morphology and glutamatergic transmission demonstrate that the loss of NLs 1-3 causes the functional shut-down of a large number of glutamatergic synapses, which develops significant decrease in SPSC and MPSC frequency, but does not influence on SPSC and mPSC amplitudes. The reason of these results obtained from respiratory brainstem of neuroligin 1-3 triple KOs could be both postsynaptic changes and presynaptic dysfunction.

\subsubsection{3 $\mathrm{Na}^{+}-, \mathrm{K}^{+}-, \mathrm{Ca}^{2+}$-channels function is not affected in NL 1-3 $\mathrm{KO}$ mice}

Testing the function of voltage-gated $\mathrm{Na}^{+}-, \mathrm{K}^{+}-, \mathrm{Ca}^{2+}$-channels in $\mathrm{PBC}$ neurons of $\mathrm{NL}$ 1-3 triple $\mathrm{KO}$ mice, we observed that neither $\mathrm{Na}^{+}$- nor $\mathrm{K}^{+}$-or $\mathrm{Ca}^{2+}$-currents or current densities were significantly altered. These data suggest that the impairment of synaptic transmission in respiratory brainstem of NL 1-3 triple KOs is not due to dysfunction of these voltage-gated channels. Similar to NL 1-3 triple $\mathrm{KO}$ mice, voltage-gated $\mathrm{Na}^{+}-, \mathrm{K}^{+}$-currents exhibited not significant changes in $\mathrm{PBC}$ neurons of $\alpha$-neurexin triple KO mice, in contrast to the voltage-dependent $\mathrm{Ca}^{2+}$-current, which was strongly reduced in $\alpha$-neurexin triple $\mathrm{KO}$ mice (Missler et al., 2003).

Thus, our data obtained from respiratory brainstem of NL 1-3 triple KO mice demonstrate that the early postnatal death of NL 1-3 triple KO mice could be a result of combination of different subtle synaptic phenotypes of the all three NL single KOs. 


\subsection{The effects of NL 2 deletion in vivo}

After examination the effects of NLs 1-3 in respiratory rhythm generating brainstem network, and finding that the loss of NLs 1-3 results more reduction of inhibitory synaptic transmission than excitatory, next we turned to analyze the inhibitory synaptic activity and synaptogenesis only in the absence of NL 2, using $\mathrm{NL} 2 \mathrm{KO}$ mice. In this study first time we show the role of NL 2 in synaptic activity in vivo. Our results demonstrate that the lack of NL 2 leads to a very strong impairment of inhibitory synaptic function and does not change the initial synapse formation in the respiratory brainstem. In contrast to a GABAregic/glycinergic synaptic activity glutamatergic synaptic transmission is not altered in absence of neuroligin 2. NL $2 \mathrm{KO}$ mice exhibit an increased ratio of glutamatergic versus GABAergic/glycinergic synapses in preBötzinger complex.

\subsubsection{Loss of NL 2 does not alter synaptogenesis in PBC}

Despite the high homology with NL 1, which is specifically localized to glutamatergic postsynaptic specializations, $\mathrm{NL} 2$ is localized to inhibitory synapses and is not recruited to gluatamtergic synapses, when NL 1 is absent. Some studies show that despite NL 2 is concentrated at inhibitory synapses, it can also associate with postsynaptic proteins from excitatory synapses, including PSD 95 (Graf et al., 2004, Chih et al., 2005). It has been shown that postsynaptic mechanisms and not interactions with presynaptic cell adhesion molecules determine the localization of NL 2 (Graf et al., 2004; Varoqueaux et al., 2004). The studies done in cultured neurons (Chih et al., 2005) revealed that overexpression and knock-down of NL 2 result substantial reduction of both excitatory and inhibitory synapse numbers, demonstrating that NL 2 , similar to other members of neuroligin family, is important regulator of synatogenesis. Our present data obtained from NL $2 \mathrm{KO}$ mice are not entirely agree with these results. Our immunofluorescence double labeling experiments in preBötzinger 
complex revealed that the number of glutamatergic and GABAergic/glycinergic pre-and postsynaptic structures was not significantly different between control and NL $2 \mathrm{KO}$ mice, as we have also seen in double labeled experiments obtained from NL 1-3 triple KO mice. Likewise, we did not observe statistically significant difference in the number of pre- and postsynaptically double labeled glutamatergic and GABAergic/glycinergic synapses in all tested genotypes. Moreover, the staining intensity was similar in control and NL KO mice. The small change that we observed was the insignificantly increased ratio between the number of glutamatergic synapses and GABAergic/glycinergic synapses in NL 2 $\mathrm{KO}$ mice, whereas other studies performed in neuronal cultures (Graf et al., 2004, Chih et al., 2005, Prange et al., 2004) demonstrate that NL 2 affects the E/I ratio. Thus, our data demonstrate that NL 2 is dispensable not only for the initial formation of glutamatergic, but also for GABAergic/glycinergic synaptic contacts in vivo.

\subsubsection{Excitatory synaptic activity remains unaltered in NL $2 \mathrm{KO}$ mice}

Our analyses of glutamatergic synaptic transmission did not reveal any functional changes in glutamatergic activity in PBC and $\mathrm{NH}$ of $\mathrm{NL} 2 \mathrm{KO}$ mice. The frequencies and amplitudes of spontaneous and miniature excitatory postsynaptic currents (sEPSCs and mEPSCs) measured in preBötzinger neurons were almost identical in control and NL 2 KO mice. Likewise, evoked glutamatergic ePSC amplitudes and failure rates were unaltered in NL 2 KOs. In addition, the responses elicited by direct application of glutamate in presence of tetrodoxin were not significantly different between control and NL 2 KO mice, and it shows that properties of excitatory postsynaptic receptors are not altered in absence of NL 2. These results together with our morphological data indicate that loss of NL 2 does not alter glutamatergic synapse numbers and glutamatergic synaptic transmission, which supports the hypothesis that other splice variants of neuroligins that are preferentially localized to glutamatergic synapses, are not recruited to inhibitory synapses, when NL 2 is absent. 


\subsubsection{Deletion of NL 2 strongly impairs GABAergic/glycinergic transmission}

As shown in our results obtained from NL 1-3 KO mice, the loss of all three neuroligins has a preferential effect on inhibitory synaptic activity. Investigating the consequences of only NL 2 deletion, we observed strong reduction of total and pharmacologically separated inhibitory synaptic activity in respiratory brainstem. The total spontaneous PSC and spontaneous inhibitory IPSC frequencies and amplitudes were strongly decreased in PBC neurons. Electrically evoked PSC and IPSC amplitudes in hyposglossal neurons were less affected in $\mathrm{NL} 2 \mathrm{KO}$ mice as compared to controls, indicating that in absence of NL 2 the synaptic activity in PBC neurons is affected stronger than in $\mathrm{NH}$. Our recordings of pharmacologically isolated GABAergic synaptic transmission and glycinergic synaptic transmission revealed that both are significantly reduced in respiratory brainstem of mice lacking NL 2. Comparison between them revealed that GABAergic SPSC frequencies and amplitudes were decreased more severe, than glycinergic SPSC frequencies and amplitudes in NL $2 \mathrm{KO}$ mice. Likewise, the deletion of NL 2 resulted stronger decrease of miniature GABAergic postsynaptic currents compared to miniature glycinergic postsynaptic currents. The large decrease in the frequency of $\mathrm{GABA}_{\mathrm{A} \text { - }}$ and glycine-receptor mediated minis in NL $2 \mathrm{KO}$ mice without a change in numbers of GABAergic/gycinergic synapses could be caused by a general defect in the function of presynaptic terminals, which can support our observation obtained in experiments involving hypertonic sucrose application. The frequency of miniature inhibitory postsynaptic currents elicited by pressure application of hypertonic sucrose was significantly decreased in PBC neurons of NL $2 \mathrm{KO}$ mice as compared to controls. The reduction of GABAergic mPSC and glycinergic mPSC amplitudes in NL 2 KO mice suggests that the decrease in mini amplitude is caused by alterations of properties of postsynaptic receptors. The direct application of $\mathrm{GABA}_{A}$-agonist muscimol during recording of miniature GABAergic postsynaptic currents elicited decreased response in NL 
$2 \mathrm{KO}$ mice compared to controls. Likewise, the response evoked by pressure applied glycine was significantly reduced in mice lacking NL 2, but less than response evoked by muscimol.

Taken together our morphological and electrophysiological data demonstrate that NL 2 is not important for initial formation of glutamatergic and GABAergic/glycinergic synaptic contacts in vivo, but it has an essential role for normal inhibitory synaptic function in intact brain, and the deletion of NL 2 impairs the GABAergic synaptic transmission stronger, than glycinergic transmission in the respiratory rhythm generating network. 


\section{Summary}

This project investigated the role of neuroligins in brainstem respiratory network of NL 1-3 and NL 2 conventional knockout mice. The deletion of all three neuroligin genes leaded to a lethal phenotype most likely due to respiratory failure, as we saw in our whole-body plethysmography recordings, whereas mice lacking only NL 2 are viable, fertile and behaviorally normal in the cage conditions. Whole-cell voltage-clamp studies on acute brainstem slices of newborn NL 1-3 KO mice, containing preBötzinger complex (PBC) and hypoglossal nucleus $(\mathrm{NH})$, showed that early postnatal death of these animals is a consequence of impaired synaptic activity in these brainstem centers that control respiration. Our detailed analyses of pharmacologically isolated glutamatergic and GABAeric/glycinergic synaptic transmission revealed dramatic decrease in GABAeric/glycinergic activity and moderately reduced glutamatergic synaptic activity in neuroligin 1-3 deficient respiratory brainstem neurons, which is not due to dysfunction of voltage-dependent $\mathrm{Ca}^{2+}$ channels, while our analyses of $\mathrm{Ca}^{2+}$ current in $\mathrm{PBC}$ neurons showed that these channels are functioning properly in NL 1-3 KO mice. In contrast to some recent works performed in neuronal cultures, where authors found that suppression of neuroligins in neuronal cultures does result in a substantial reduction of excitatory and inhibitory synapse numbers, and this change consequently alters synaptic transmission in these neurons, we observed unaltered symmetric and asymmetric synapse numbers in mice lacking all three neuroligins. Interestingly, NL 1-3 triple KOs exhibit an increased E/l ratio and as known, several neurobiological disorders, such as a autism is thought to be caused by an increase in the E/I ratio, and considering the potential role of neuroligins in dictating the E/l ratio, neuroligin knockout mice could serve as a useful animal model of neurological diseases.

Regarding the fact that the loss of neuroligins affected inhibitory synaptic transmission more strongly than excitatory, we next investigated in detail the role 
NL 2, which is preferentially localized at inhibitory synapses. As it was expected, similar to data obtained from NL 1-3 KO mice neither the excitatory nor inhibitory

synapse number was changed in NL 2 KO PBC neurons. Electrophysiological analyses revealed unaltered glutamatergic and dramatically reduced GABAergic/glycinergic transmission. Pharmacological separation of the GABAergic and glycineric postsynaptic currents showed that both were significantly decreased in NL 2 deficient mice, but the deletion of NL 2 caused a stronger impairment of GABAergic synaptic transmission, than glycinergic synaptic activity.

Taken together, our results indicate that neuroligins are not required for initial synapse formation, but are essential for proper synapse maturation and brain function, and the loss of single NL 2 impairs GABAergic/glycinergic synaptic function, which is not compensated in the presence of other members of neuroligin family. 


\section{References}

Aylward EH, Minshew NJ, Goldstein G, Honeycutt NA, Augustine AM, Yates KO, Barta PE, Pearlson GD (1999) MRI volumes of amygdala and hippocampus in non-mentally retarded autistic adolescents and adults. Neurology 53: 2145-50.

Beaudet, AL (2002) Is medical genetics neglecting epigenetics? Genet Med. 4: 399-402.

Bolliger MF, Frei K, Winterhalter KH, Gloor SM (2001) Identification of a novel neuroligin in humans which binds to PSD-95 and has a widespread expression. Biochem. J. 356: 581-588.

Boucard AA, Chubykin AA, Comoletti D, Taylor P, Südhof, TC (2005) A splice code for trans-synaptic cell adhesion mediated by binding of neuroligin 1 to alpha- and beta-neurexins. Neuron 48: 229-236.

Brose N (1999) Synaptic cell adhesion proteins and synaptogenesis in the mammalian central nervous system. Naturwissenschaften 86: 516-524.

Connelly CA, Dobbins EG, Feldman JL (1992) Pre-Bötzinger complex in cats: respiratory neuronal discharge patterns. Brain Res. 590: 337-340.

Chih B, Afridi SK, Clark L, Scheiffele P (2004) Disorder-associated mutations lead to functional inactivation of neuroligins. Hum. Mol. Genet. 13: 14711477.

Chih B, Engelman H, Scheiffele P (2005) Control of excitatory and inhibitory synapse formation by neuroligins. Science 307: 1324-1328.

Chih B, Gollan L, Scheiffele P (2006) Alternative splicing controls selective transsynaptic interactions of the neuroligin-neurexin complex. Neuron $51,: 171-$ 178. 
Chubykin AA, Liu, X, Comoletti D, Tsigelny I, Taylor P, Südhof, T.C. (2005) Dissection of synapse induction by neuroligins: Effects of a neuroligin mutation associated with autism. J. Biol. Chem. 280: 22365-22374.

Comoletti D, De Jaco A, Jennings LL, Flynn RE, Gaietta G, Tsigelny I, Ellisman MH, Taylor P (2004) The Arg451Cysneuroligin-3 mutation associated with autism reveals a defect in protein processing. J. Neurosci. 24: 4889-4893.

Craig AM, Ethan R, Linhoff G, Linhoff W (2006) How to build a central synapse: clues from cell culture. Trends Neurosci. 29: 8-20.

Dean C, Scholl FG, Choih J, De Maria S, Berger J, Isacoff E, Scheiffele P (2003).Neurexin mediates the assembly of presynaptic terminals. Nat. Neurosci. 6: 708-716.

Dean C, Dresbach T (2006) Neuroligins and neurexins: linking cell adhesion, synapse formation and cognitive function. Trends Neurosci. 29: 21-29.

Dresbach T, Qualmann B, Kessels MM, Garner CC, Gundelfinger ED (2001)The presynaptic cytomatrix of brain synapses. Cell Mol Life Sci. 58: 94-116.

Feldman JL, Mitchell GS, Nattie EE (2003) Breathing: rhythmicity, plasticity, chemosensitivity. Ann. Rev. Neurosci 26: 239-266.

Garner, CC, Zhai RG, Gundelfinger ED, Ziv NE (2002) Molecular mechanisms of CNS synaptogenesis. Trends Neurosci. 25: 243-251.

Gilbert MM, Auld VJ (2005) Evolution of clams (cholinesterase-like adhesion molecules): structure and function during development. Front. Biosci. 10: 2177-2192.

Graf ER, Zhang X, Jin SX. Linhoff MW, Craig, AM (2004) Neurexins induce differentiation of GABA and glutamate postsynaptic specializations via neuroligins. Cell 119: 1013-1026. 
Gray PA, Rekling, JC, Bocchiaro CM, Feldman JL (1999) Modulation of respiratory frequency by peptidergic input to rhythmogenic neurons in preBötzinger complex. Science 286: 1566-1568.

Guyenet, PG, Wang H (2001) Pre-Bötzinger neurons with preinspiratory discharges "in vivo" express NK1 receptors in the rat. J. Neurophysiol. 86: 438-446.

Harlow ML, Ress D, Stoschek A, Marshall RM, McMahan UJ (2001) The architecture of active zone material at the frog's neuromuscular junction. Nature 409: 479-84.

Herbert MR (2005) Large brains in autism: the challenge of pervasive abnormality. Neuroscientist 11: 417-440.

Hirao K, Hata Y, Ide N, Takeuchi M, Irie M, Yao I, Deguchi M, Toyoda A, Südhof TC, Takai Y. (1998) A novel multiple PDZ domain-containing molecule interacting with $\mathrm{N}$-methyl-D-aspartate receptors and neuronal cell adhesion proteins. J. Biol. Chem. 273: 21105-21110.

Hussman JP (2001) Suppressed GABAergic inhibition as a common factor in suspected etiologies of autism. J Autism Dev Disord 31: 247-248.

Ichtchenko K, Hata Y, Nguyen T, Ullrich B, Missler M, Moomaw C, Südhof TC (1995) Neuroligin 1: a splice site-specific ligand for beta-neurexins. Cell 81: 435-443.

Ichtchenko K, Nguyen T, Südhof TC (1996) Structures, alternative splicing, and neurexin binding of multiple neuroligins. J. Biol. Chem. 271: 2676-2682.

Irie M, Hata Y, Takeuchi M, Ichtchenko K, Toyoda A, Hirao K, Takai Y, Rosahl TW, Südhof TC (1997). Binding of neuroligins to PSD-95. Science 277: 1511-1515. 
Jamain S, Quach H, Betancur C, Rastam M, Colineaux C, Gillberg IC, Soderstrom H, Giros B, Leboyer M, Gillberg C, Bourgeron T (2003) Mutations of the $X$-linked genes encoding neuroligins NLGN3 and NLGN4 are associated with autism. Nat. Genet. 34: 27-29.

Kim E, Sheng M. (2004) PDZ domain proteins of synapses. Nat. Rev. Neurosci. 5: $771-781$

Landis DM, Hall AK, Weinstein LA, Reese TS (1988) The organisation of cytoplasm at the presynaptic active zone of a central nervous system synapse. Neuron 1: 201-209

Laumonnier F, Bonnet-Brilhault F, Gomot M, Blanc R, David A, Moizard MP, Raynaud M, Ronce N, Lemonnier E, Calvas P (2004) X-linked mental retardation and autism are associated with a mutation in the NLGN4 gene, a member of the neuroligin family. Am. J. Hum. Genet. 74: 552-557.

Levinson JN, Chery N, Huang K, Wong TP, Gerrow K, Kang R, Prange O, Wang YT, El-Husseini A (2005) Neuroligins mediate excitatory and inhibitory synapse formation: involvement of PSD-95 and neurexin-1beta in neuroligin-induced synaptic specificity. J. Biol. Chem. 280: 17312-17319.

Li Z, Sheng M (2003) Some assembly required: the development of neuronal synapses. Nat. Rev. Mol. Cell Biol. 4: 833-841.

Lise MF, El-Husseini A (2006) The neuroligin and neurexin families: from structure to function at the synapse. Cell. Mol. Life Sci. 63: 1833-1849.

Meyer G, Varoqueaux F, Neeb A, Oschlies M, Brose N (2004) The complexity of PDZ domain-mediated interactions at glutamatergic synapses: a case study on neuroligin. Neuropharmacology 47: 724-733.

Missler M, Zhang W, Rohlmann A, Kattenstroth G, Hammer RE, Gottmann K, Südhof, TC (2003) Alpha-neurexins couple Ca2+ channels to synaptic vesicle exocytosis. Nature 423: 939-948. 
Mohrmann R, Werner M, Hatt H, Gottmann K (1999) Target-specific factors regulate the formation of glutamatergic transmitter release sites in cultured neocortical neurons. J. Neurosci. 19: 10004-10013.

Montgomery JM, Zamorano PL, Garner CC (2004) MAGUKs in synapse assembly and function: an emerging view. Cell. Mol. Life Sci. 61: 911-929.

Nam Cl, Chen L (2005) Postsynaptic assembly induced by neurexin-neuroligin interaction and neurotransmitter. Proc. Natl. Acad. Sci. USA 102: 61376142.

Nguyen T, Sudhof TC (1997) Binding properties of neuroligin 1 and neurexin 1 beta reveal function as heterophilic cell adhesion molecules. J. Biol. Chem. 272: 26032-26039.

Paraoanu LE, Becker-Roeck M, Christ E, Layer PG (2005) Expression patterns of neurexin-1 and neuroligins in brain and retina of the chick embryo: neuroligin-3 is absent in retina. Neurosci. Lett. 395: 114-117.

Philibert R A, Winfield SL, Sandhu HK, Martin BM, Ginns, El (2000) The structure and expression of the human neuroligin-3 gene. Gene 246: 303-310.

Pierrefiche O, Schwarzacher S.W, Bischoff AM, Richter DW (1998) Blockade of synaptic inhibition within the pre-Bötzinger complex in the cat suppresses respiratory rhythm generation in vivo. J. Physiol. 509: 245-254.

Prange O, Wong TP, Gerrow K, Wang YT, El-Husseini A (2004) A balance between excitatory and inhibitory synapses is controlled by PSD-95 and neuroligin. Proc. Natl. Acad. Sci. USA 101: 13915-13920.

Rao A, Harms KJ, Craig AM (2000) Neuroligation: building synapses around the neurexin-neuroligin link. Nat Neurosci. 3: 747-749.

Richter DW, Spyer KM (2001) Studying rhythmogenesis of breathing: comparison of in vivo and in vitro models. Trends Neurosci 24: 464-472. 
Ritter B, Zhang W (2000) Early postnatal maturation of $\mathrm{GABA}_{A}$-mediated inhibition in the brainstem respiratory rhythmgenerating network of the mouse. Eur. J. Neurosci. 12: 2975-2984.

Scheiffele P (2003) Cell-cell signaling during synapse formation in the CNS. Annu. Rev. Neurosci. 26: 485-508.

Scheiffele P, Fan J, Choih J, Fetter R, Serafini T (2000) Neuroligin expressed in nonneuronal cells triggers presynaptic development in contacting axons. Cell 101: 657-669.

Schlüter M, Schmitz F, Reinhard J, Rosenmund, C, Südhof TC (2004). A complete genetic analysis of neuronal Rab3 function. J. Neurosci. 24: 6629-6637.

Schwarzacher SW, Smith JC, Richter DW (1995) Pre-Bötzinger complex in the cat. J.Neurophysiol. 73: 1452-1461.

Serafini, T. (1999) Finding a partner in a crowd: neuronal diversity and synaptogenesis. Cell 98: 133-136.

Song JY, Ichtchenko K, Südhof TC, Brose N (1999) Neuroligin 1 is a postsynaptic cell-adhesion molecule of excitatory synapses. Proc. Natl. Acad. Sci. USA 96: 1100-1105.

Smith JC, Ellenberger HH, Ballanyi K, Richter DW, Feldman JL (1991) PreBötzinger complex: a brainstem region that may generate respiratory rhythm in mammals. Science 254: 726-729.

Solomon IC, Edelman NH, Neubauer JA (1999) Patterns of phrenic motor output evoked by cchemical stimulation of neurons located in the pre-Bötzinger complex in vivo. J. Neurophysiol. 81: 1150-1161.

Talebizadeh Z, Lam DY, Theodoro MF, Bittel DC, Lushington GH, Butler MG (2006) Novel splice isoforms for NLGN3 and NLGN4 with possible implications in autism. J. Med. Genet. 43, e21. 
Turrigiano GG, Nelson SB (2004) Homeostatic plasticity in the developing nervous system. Nat. Rev. Neurosci. 5: 97-107.

Varoqueaux F, Jamain S, Brose N. (2004) Neuroligin 2 is exclusively localized to inhibitory synapses. Eur. J. Cell Biol. 83: 449-456.

Wang H, Stornetta RL, Rosin DL, Guyenet PG (2001) Neurokinin-1 receptorimmunoreactive neurons of the ventral respiratory group in the rat. J. Comp. Neurol. 434: 128-46.

Yamagata M, Sanes JR, Weiner JA (2003) Synaptic adhesion molecules. Curr. Opin. Cell Biol. 15: 621-632.

Yan J, Oliveira G, Coutinho A, Yang C, Feng J, Katz C, Sram J, Bockholt A, Jones IR, Craddock N, Cook EH, Vicente A, Sommer SS (2005) Analysis of the neuroligin 3 and 4 genes in autism and other neuropsychiatric patients. Mol. Psychiatry 10: 329-332.

Zhang W, Elsen F, Barnbrock A, Richter DW (1999) Postnatal development of $\mathrm{GABA}_{\mathrm{B}}$ receptor-mediated modulation of voltage-activated $\mathrm{Ca}^{2+}$ - currents in mouse brainstem neurons. Eur J Neurosci. 11: 2332-42.

Zhang W, Rohlmann A, Sargsyan V, Aramuni G, Hammer RE, Südhof TC, Missler M (2005) Extracellular domains of alphaneurexins participate in regulating synaptic transmission by selectively affecting $\mathrm{N}$ - and $\mathrm{P} / \mathrm{Q}$-type Ca2+ channels. J. Neurosci. 25: 4330-4342.

Zoghbi HY (2003) Postnatal neurodevelopmental disorders: meetings at the synapses? Science 302: 826-830.

Zucker RS (1993) Calcium and transmitter release. J Physiol Paris; 87: 25-36. 


\section{Acknowledgments}

I would like to thank to my supervisor Priv.-Doz. Dr. Weiqi Zhang for his support and encouragement throughout this project and for giving me the opportunity to work in his laboratory. I express my sincere thanks to Prof. Dr. Ralf Heinrich for agreeing to be the referent and main examiner for my thesis. I am very thankful to Prof. Dr. Michael Hörner for granting permission to be the co-referent of my thesis. I would like deeply to thank to Prof. Dr. R. Ficner and Priv.-Doz. Dr. M. Hoppert for being my examiners; Prof. Dr. Fritz and Prof. Dr. C. Gatz for agreeing to be the Reviewers for my thesis. Sincere thanks to Prof. Dr. Diethelm W. Richter, Head of the Department for all the support during my research. I am indebted to Prof. Dr. Nils Brose and Dr. F. Varoqueaux for providing me the opportunity to work on this interesting project and for the great help and support they provided.

I would like to thank to Lila Tantalaki for nice time in daily life and for the help in the lab. Many thanks to Alicia Stradomska, Lucian Medrihan for being a good friends and making my life in the lab more interesting. I am thankful to Andreas Bock for his support with official matters and for the great help with the final preparation of the thesis. Many thanks to Tobias Schwarz, Olivie Bidon for being friendly colleagues and always helping me to solve the problems during some experiments. I would like to thank to Peter Frank and Wayne Sidio for their help with computers. Thanks to Saju Balakrishnan for friendly conversations and helping me with the thesis submission procedures. Special thanks to Cornelia Hühne for her deep friendship and support. Thanks a million to Vard Sargsyan for her care, countless helps, motivation and scientific discussions. Big thanks to little Seda and Sona for spreading happiness around me. Many thanks to all colleagues from Physiology institute for creating pleasant work atmosphere. Thanks very much too to all my friends, who made my life amazingly wonderful during my research in Göttingen. 
I express my biggest gratitude to my dear parents, Sylva Daghunc, Senikerim Aramuni, and my brother Gevorg Aramuni for their endless love, care and enormous support. 


\section{Curriculum Vitae}

Gayane Aramuni

Am Vogelsang 1

37075 Göttingen

Tel: (+49172)1593593

e-mail:garamun1@gwdg.de

aramuni2001@yahoo.com

\section{PERSONAL DATA}

Date of Birth

Place of Birth

Nationality

Sex

Degree
05.04.1977

Echmiadzin, Armavir, Armenia

Armenian

Female

M.Sc

\section{EDUCATION}

1995 - 1999

Yerevan State University, Department of Biology Yerevan, Armenia

B.Sc

1999 - 2001

Yerevan State University, Department of Biology Yerevan, Armenia

M.Sc.

March 2003 to date

Centre Physiology and Pathophysiology

Department of Neurophysiology, University of

Göttingen

PhD student

2001 - 2003

Yerevan State University, Department of Biology

Yerevan, Armenia

Scientific researcher

$2001-2003$

Secondary school N1

Echmiadzin, Armenia

Teacher of Biology and Chemistry 


\section{Publications}

\section{Articles and manuscripts}

Varoqueaux $\mathrm{F}^{*}$, Aramuni $\mathbf{G}^{*}$, Rawson RL, Mohrmann R, Missler M, Gottmann, Zhang W, Südhof TC, Brose N (2006) Neuroligins determine synapse maturation and function. Neuron 51: 1-14 ( ${ }^{*}$ Varoqueaux F and Aramuni G contributed equally to this work).

Zhang W, Rohlmann A, Sargsyan V, Aramuni G, Hammer R.E, Südhof TC, Missler M, (2005) Extracellular domains of $\alpha$ - neurexins participate in regulating synaptic transmission by selectively affecting $\mathrm{N}$ - and P/Q-type $\mathrm{Ca}^{2+}$ channels. J. Neurosci. 25: 4330-4342.

Aramuni G, Varoqueaux F, Sargsyan V, Brose N, Zhang W (2007) Neuroligin 2 regulates inhibitory synaptic transmission. Manuscript in preparation.

Aramuni G, Sargsyan V, Zhang W (2007) Mutual modulation of $\mu$-opioid and dopamine 1 receptors in respiratory network of mouse. Manuscript in preparation.

Medrihan L, Tantalaki E, Sargsyan V, Aramuni G, Dudanova I, Missler M, Zhang W (2007) Early postnatal changes in GABA receptor-mediated synaptic transmission in the MeCP2 mouse model of Rett syndrome. Manuscript

Sieber M, Martinez de la Torre M, Müller T, Vasyutina E, Dutschmann M, Aramuni G, Zhang W, Cemil Özcelik, Birchmeier C (2007) The homeodomain factor Lbx1 controls the differentiation of sensory interneurons in the hindbrain. Manuscript

Matzke A, Sargsyan V, Aramuni G, Holtmann B, Sendtner M, Pace G, Howells $\mathrm{N}$, Huber S, Ponta H, Zhang W, Orian-Rousseau V (2007) CD44 is required for c-Met function in vivo. Manuscript 
Sargsyan V, Aramuni G, Zhang W (2007) The modulation of neuronal pacemaker channels by $\mathrm{GABA}_{B}$ receptor in brainstem respiratory network is developmentaly regulated and involves distinct signalling pathways. Manuscript in preparation

Sargsyan V, Stettner GM, Aramuni G, Bettler B, Dutschmann M, Zhang W (2007) $G_{A B A_{B}}$ receptors are not involved in breathing, but are involved in gasping. Manuscript in preparation

\section{Posters}

G. Aramuni, F. Varoqueaux, V. Sargsyan, N. Brose and W.Zhang (2006) The Role of neuroligin 2 in regulation of inhibitory synaptic transmission. FENS Abstr., vol.3, A119.2, 2006.

G. Aramuni, V. Sargsyan and Zhang, W. (2006) Mutual modulation of $\mu$-opioid and dopamine 1 receptors in developing respiratory network of mouse. Acta Physiologica 186 (Suppl. 1): p.133

V. Sargsyan, G. Aramuni and W. Zhang (2006) Biphasic effects of $G_{A B A_{B}}$ receptor on $I_{h}$ currents in developing respiratory network of mouse. Acta Physiologica 186 (Suppl. 1): p. 131

Aramuni G, Varoqueaux F, Sargsyan V, Brose N and Zhang W (2005) Neuroligins are essential for neuronal network function in respiratory network of mouse. Proceedings of the 6th Meeting of the German Neuroscience Society Society 2005, eds: H. Zimmermann and K. Kriglstein, Neurophorum 2005, 1 Suppl 295A

Sargsyan, V., Aramuni, G. and Zhang, W. (2005) The role of protein-protein interaction in mutual regulation of the function of $G A B A_{A}$ and $G A B A_{B}$ receptors during postnatal development. Proceedings of the 6th Meeting of the German Neuroscience Society 2005, eds: H. Zimmermann and K. Kriglstein, Neurophorum 2005, 1 Suppl 324B 
Tantalaki, E., Aramuni, G. and Zhang, W. (2005) GABA $_{B}$-receptor-mediated signaling changes during early postnatal development in brainstem of mouse. Pflügers Archiv 449 (Suppl. 1): S86

Aramuni, G., Sargsyan, V., Missler, M. and Zhang, W. (2004) a-neurexins organize presynaptic terminals by coupling synaptic cell adhesion to $\mathrm{Ca}^{2+}$ channel function. Pflügers Archiv 447 (Suppl. 1): S28

Tantalaki, E., Sargsyan, V., Aramuni, G. and Zhang, W. (2004) Impairments of $\mathrm{GABA}_{B}$-mediated modulation of $\mathrm{Ca}^{2+}$ channel in $\alpha$-neurexin mutant mice. Pflügers Archiv 447 (Suppl.1): S125 